UCLA/11/TEP/110

SLAC-PUB-14529
SU-ITP-11/34

NSF-KITP-11-236
Saclay-IPhT-T11/175

CERN-PH-TH/2011/190

\title{
Simplifying Multiloop Integrands and Ultraviolet Divergences of Gauge Theory and Gravity Amplitudes
}

\author{
Z. Bern ${ }^{a, b, c}$, J. J. M. Carrasco ${ }^{b, d}$, L. J. Dixon ${ }^{c, e}$, H. Johansson ${ }^{b, f}$ and R. Roiban ${ }^{b, g}$ \\ ${ }^{a}$ Department of Physics and Astronomy, UCLA, Los Angeles, CA 90095, USA \\ ${ }^{b}$ Kavli Institute for Theoretical Physics, University of California, Santa Barbara, CA 93106, USA \\ ${ }^{c}$ Theory Group, Physics Department, CERN, CH-1211 Geneva 23, Switzerland \\ ${ }^{d}$ Stanford Institute for Theoretical Physics and Department of Physics, \\ Stanford University, Stanford, CA 94305, USA \\ ${ }^{e}$ SLAC National Accelerator Laboratory, Stanford University, Stanford, CA 94309, USA \\ ${ }^{f}$ Institut de Physique Théorique, CEA-Saclay, F-91191 Gif-sur-Yvette cedex, France \\ ${ }^{g}$ Department of Physics, Pennsylvania State University, University Park, PA 16802, USA
}

\begin{abstract}
We use the duality between color and kinematics to simplify the construction of the complete four-loop four-point amplitude of $\mathcal{N}=4$ super-Yang-Mills theory, including the nonplanar contributions. The duality completely determines the amplitude's integrand in terms of just two planar graphs. The existence of a manifestly dual gauge-theory amplitude trivializes the construction of the corresponding $\mathcal{N}=8$ supergravity integrand, whose graph numerators are double copies (squares) of the $\mathcal{N}=4$ super-Yang-Mills numerators. The success of this procedure provides further nontrivial evidence that the duality and double-copy properties hold at loop level. The new form of the four-loop four-point supergravity amplitude makes manifest the same ultraviolet power counting as the corresponding $\mathcal{N}=4$ super-Yang-Mills amplitude. We determine the amplitude's ultraviolet pole in the critical dimension of $D=11 / 2$, the same dimension as for $\mathcal{N}=4$ super-YangMills theory. Strikingly, exactly the same combination of vacuum integrals (after simplification) describes the ultraviolet divergence of $\mathcal{N}=8$ supergravity as the subleading-in- $1 / N_{c}^{2}$ single-trace divergence in $\mathcal{N}=4$ super-Yang-Mills theory.
\end{abstract}

PACS numbers: 04.65.+e, 11.15.Bt, 11.30.Pb, 11.55.Bq 


\section{INTRODUCTION}

The past few years have brought remarkable advances in understanding scattering amplitudes in the maximally supersymmetric $\mathcal{N}=4$ super-Yang-Mills (sYM) theory [1] in the planar limit of a large number of colors. It may soon be possible to completely determine all planar scattering amplitudes in this theory, for all values of the coupling, going far beyond the (now thoroughly understood) cases of four and five external gluons [2]. Much of this progress has been surveyed recently [3]. Planar scattering amplitudes exhibit a new symmetry known as dual conformal symmetry [4, 5], which severely restricts their structure. Together with supersymmetry and (position space) conformal symmetry, dual conformal invariance gives rise to a Yangian [6] - an algebraic structure common in integrable models. Indeed, it is widely believed that several aspects of the planar sector of $\mathcal{N}=4 \mathrm{sYM}$ theory are controlled by an integrable model (see e.g. ref. [7]).

In contrast, much less is known about the nonplanar sector - the subject of the present paper. Consider $\mathcal{N}=4 \mathrm{sYM}$ theory for the gauge group $S U\left(N_{c}\right)$. In the limit $N_{c} \rightarrow \infty$, the nonplanar, or subleading-color, contributions are suppressed by powers of $1 / N_{c}$. Once one takes into account these corrections, for finite $N_{c}$, the scattering amplitudes no longer appear to possess dual conformal symmetry, nor do they demonstrate any obvious integrability properties.

Understanding the subleading-color terms is critical to a complete description of the behavior of gauge theories. For example, many types of color correlations are suppressed in the large- $N_{c}$ limit. Furthermore, the information provided by the full-color expression for $\mathcal{N}=4 \mathrm{sYM}$ amplitudes, expressed in terms of their loop-momentum integrands, can be used to construct corresponding amplitudes [8-11] in $\mathcal{N}=8$ supergravity [12]. From each set of amplitudes one can extract information about ultraviolet divergences in the respective theory.

The ultraviolet (UV) properties of $\mathcal{N}=8$ supergravity have been the focus of intense investigation. There have been several recent reviews of the situation [13]. Long ago, an $\mathcal{N}=8$ supersymmetric local counterterm at three loops in $D=4$ was proposed [1418]. An explicit computation of the three-loop four-graviton amplitude first revealed that the counterterm has a vanishing coefficient [9]. Subsequently it was realized [19] that this counterterm is forbidden in $D=4$ by the $E_{7(7)}$ duality symmetry [12]. Other analyses have extended the finiteness constraints from $E_{7(7)}$ and linearized supersymmetry, such that the first potential divergence in $D=4$ is now at seven loops [20-23]. Finiteness until this loop order happens to agree with an earlier naive power-counting, based on the assumption of an off-shell $\mathcal{N}=8$ superspace [24]. A potential seven-loop divergence is also suggested by other approaches, including an analysis of string theory dualities [25], a first-quantized world-line approach [26], and light-cone supergraphs [27]. However, it has also been argued that the theory may remain finite beyond seven loops [28].

In this paper, we will show how a conjectured duality between color and kinematics [29, 30] provides a powerful method for computing subleading-color terms in $\mathcal{N}=4 \mathrm{sYM}$ amplitudes, in a way that makes the construction of the corresponding $\mathcal{N}=8$ supergravity amplitudes extremely simple. Also, the $\mathcal{N}=8$ result is expressed in a form that makes manifest the true ultraviolet behavior of the amplitude (when continued to higher space-time dimension $D)$. Thus this method provides unprecedented access to the precise coefficients of potential counterterms in $\mathcal{N}=8$ supergravity, as well as in its higher-dimensional versions. It may eventually offer a means for settling the question of whether additional UV cancellations exist 
in $\mathcal{N}=8$ supergravity, beyond the known or expected ones. Perhaps even more importantly, the method gives a means for constructing complete amplitudes, allowing for detailed studies of their symmetries and properties.

A key point is that when the color-kinematics duality holds manifestly, it locks the nonplanar contributions to the planar ones. The nonplanar contributions are essential for evaluating gravity amplitudes, because in gravity theories no separation exists between planar and nonplanar contributions. This duality allows one to efficiently export information from the planar sector, e.g. that provided by dual conformal symmetry, to the much more intricate nonplanar sector.

A second key point is the claim [30] that if a duality-respecting representation of $\mathcal{N}=4$ sYM amplitudes can be found, then the loop-momentum integrands of the corresponding $\mathcal{N}=8$ supergravity amplitudes can be obtained simply by taking the graphs of $\mathcal{N}=4 \mathrm{sYM}$ theory, dropping the color factors and squaring their kinematic numerators. This doublecopy property is a loop-level generalization of the corresponding tree-level property [29], equivalent to the Kawai-Lewellen-Tye (KLT) relations between gravity and gauge-theory amplitudes [31]. Using the color-kinematics duality, followed by the double-copy property, advances in constructing integrands for the planar sector of gauge theory can be carried over to the nonplanar sector, and then on to gravity. The color-kinematic duality and the gravity double-copy property do not appear to require supersymmetry, although amplitudes in supersymmetric theories are generally much simpler to work with than non-supersymmetric amplitudes. Another important aspect of the duality is that it appears to hold in any dimension, thus making it compatible with dimensional regularization.

In this paper we will exploit the color-kinematic duality to construct the complete fourloop four-point amplitudes of $\mathcal{N}=4 \mathrm{sYM}$ theory and $\mathcal{N}=8$ supergravity. Both amplitudes were constructed previously by us $[10,11]$; however, the present construction is considerably more efficient, and makes various properties of the amplitudes more manifest. The colorkinematic duality relations allow us to express the four-loop loop-momentum integrands, for 83 different cubic (trivalent) graphs, as functionals of the integrands of just two planar graphs. For the one-, two- and three-loop four-point [30, 32], and the one- and two-loop five-point cases [33], the duality is even more restrictive: a single planar graph suffices to determine all the others. As it is becoming increasingly simple to construct planar amplitudes, a particularly attractive aspect of using the color-kinematic duality is that it determines nonplanar contributions from planar ones. Perhaps even more remarkable, in terms of measuring the redundancy found in local gauge-theory scattering amplitudes, we shall find that the entire non-trivial dynamical information in the four-loop four-point amplitude is contained in a single nonplanar graph; all other graphs are related to this one by the duality.

While a general proof of the duality conjecture is yet to be given beyond tree level, the four-loop construction we offer in this paper provides further evidence in favor of it, in the form of a highly nontrivial example. In this work, we have confirmed the dualitybased construction by verifying that the integrand matches a complete (spanning) set of generalized unitarity cuts.

Based on the double-copy structure of supergravity amplitudes, we will give a new representation of the four-loop four-point $\mathcal{N}=8$ supergravity amplitude. This construction provides a direct multiloop confirmation of the double-copy property, because we verify the generalized unitarity cuts for the new form of the supergravity amplitude, against the cuts of the known expression [10], originally constructed using the KLT relations. We also explore 
the ultraviolet properties of the amplitude in $D>4$ dimensions. An essential feature of the new representation is that the UV behavior is manifest: Individual integrals diverge logarithmically in precisely the same critical dimension $D_{c}$ as their sum. This property did not hold for the previous form of the amplitude [10]. The critical dimension is also the same as that for the planar and single-trace sectors of $\mathcal{N}=4 \mathrm{sYM}$ theory. In a previous paper [10], we showed that the supergravity amplitude is finite for $D<11 / 2$, which is also the bound obeyed by $\mathcal{N}=4 \mathrm{sYM}$ theory. However, the previous form of the amplitude did not display this bound manifestly. To see the cancellation of potential UV divergences, the integrals had to be expanded a few orders in powers of the external momenta. The lack of manifest UV behavior in that representation made it difficult to carry out the required integration in $D=11 / 2$ and to determine whether the amplitude actually does diverge in this dimension. With the new form, this task is greatly simplified, allowing us to carry it out here.

Due to the double-copy construction, the numerators of the integrands for the $\mathcal{N}=8 \mathrm{su}$ pergravity amplitude are perfect squares. However, they multiply propagator denominators that do not have definite signs. Therefore, individual integrals contributing to the amplitude can have different signs. To probe whether or not the four-loop amplitude diverges in $D=11 / 2$, it is necessary to actually evaluate the UV divergences in the loop integrals in this dimension. Using the double-copy form of the four-loop four-point amplitude we do so, finding that the $\mathcal{N}=8$ finiteness bound is in fact saturated at $D=11 / 2$ at four loops, which matches the behavior of $\mathcal{N}=4 \mathrm{sYM}$ theory. Moreover, we calculate the precise coefficient of the $\mathcal{N}=8$ supergravity divergence. We find that it exactly matches the coefficient of the divergence of the $1 / N_{c}^{2}$-suppressed single-trace term in the four-loop four-point amplitude of $\mathcal{N}=4 \mathrm{sYM}$ theory, up to an overall rational factor. Although this property is most striking at four loops, only emerging after a number of simplifications, it is consistent with lowerloop behavior. Presumably this consistent connection is a clue for unraveling the general UV properties of $\mathcal{N}=8$ supergravity.

Regularization is a crucial point in the construction of loop-level amplitudes in massless theories, because such amplitudes are usually either infrared or UV divergent. The issue of regularization has been studied in some detail in the context of unitarity cuts in ref. [34], where the six-dimensional helicity formalism [35] was suggested as a general means for implementing either dimensional regularization [36] or a massive infrared regulator equivalent to the one in ref. [37]. In the present paper we take advantage of an earlier construction of the four-loop four-point amplitude of $\mathcal{N}=4 \mathrm{sYM}$ theory, which provides expressions with demonstrated validity for $D \leq 6[11,34]$. In this paper, we compare the $D$-dimensional unitarity cuts of the new results with the cuts of the earlier results. We find exact agreement, confirming the new representations.

This paper is organized as follows. In section II we explain our strategy for constructing multiloop integrands, illustrating it with the three-loop four-point $\mathcal{N}=4 \mathrm{sYM}$ amplitude. In section III (and appendix B) we present the new forms of the four-loop integrands of $\mathcal{N}=4$ sYM theory and $\mathcal{N}=8$ supergravity. We also outline their construction. In section IV, we obtain the explicit value of the UV divergence of the $\mathcal{N}=8$ supergravity amplitude in $D=$ $11 / 2$ and discuss its properties. We also determine the UV divergence of the color doubletrace terms in the four-loop $\mathcal{N}=4 \mathrm{sYM}$ amplitude in $D=6$. (We had found earlier [11] that the double-trace divergence canceled in the next possible lower dimension, $D=11 / 2$.) We give our conclusions and outlook in section V. Several appendices are included. The first one gives functional defining relations between the numerators in the four-loop fourpoint amplitude, which are derived from the Jacobi relations after imposing some auxiliary 
conditions valid for $\mathcal{N}=4$ sYM theory. Appendix B presents the analytic expressions for the numerators. Appendix $\mathrm{C}$ gives the values of the vacuum integrals entering the UV divergence for the supergravity amplitude in the critical dimension, as well as expressions for the vacuum integrals' numerators. Explicit expressions for the color factors for each contribution to the full four-loop amplitude may be found online [38], where we also provide plain-text, computer-readable versions of the numerator factors and the kinematic dual Jacobi relations that they obey.

\section{CONSTRUCTING MULTILOOP INTEGRANDS}

The unitarity method [11, 39-41] has become a general-purpose tool for constructing multiloop amplitudes in gauge and gravity theories. In this section we demonstrate how one can dramatically reduce the complexity of unitarity-based calculations for gauge theories by assuming the conjectured duality between color and kinematics [29, 30]. This duality reduces the construction of an amplitude at the integrand level to the determination of the numerator factors for a small set of graphs, which we call "master graphs". For the four-loop four-point $\mathcal{N}=4 \mathrm{sYM}$ amplitude, it suffices to use just two planar master graphs. Alternatively, a single nonplanar master graph is sufficient.

Given the duality-satisfying form of the $\mathcal{N}=4$ sYM amplitude, the $\mathcal{N}=8$ supergravity amplitude can be written down immediately by squaring the $\mathcal{N}=4$ sYM numerator factors. We confirm the correctness of the derived gauge and gravity amplitudes using a spanning set of generalized unitarity cuts, showing that they agree with our previous forms [10, 11] on these cuts.

\section{A. Duality between color and kinematics}

In general, a massless $m$-point $L$-loop gauge-theory amplitude $\mathcal{A}_{m}^{(L)}$ in $D$ space-time dimensions, with all particles in the adjoint representation, may be written as

$$
\mathcal{A}_{m}^{(L)}=i^{L} g^{m-2+2 L} \sum_{i \in \Gamma} \int \prod_{l=1}^{L} \frac{d^{D} p_{l}}{(2 \pi)^{D}} \frac{1}{S_{i}} \frac{n_{i} C_{i}}{\prod_{\alpha_{i}} p_{\alpha_{i}}^{2}},
$$

where $g$ is the gauge coupling constant. The sum runs over the complete set $\Gamma$ of $m$-point $L$ loop graphs with only cubic (trivalent) vertices, including all permutations of external legs. In each term, the product in the denominator runs over all propagators of the corresponding cubic graph. The integrations are over the independent loop momenta $p_{l}$. The coefficients $C_{i}$ are the color factors obtained from the gauge-group structure constants by dressing every three-vertex in the graph with a factor

$$
\tilde{f}^{a b c}=i \sqrt{2} f^{a b c}=\operatorname{Tr}\left(\left[T^{a}, T^{b}\right] T^{c}\right),
$$

where the hermitian generators of the gauge group are normalized via $\operatorname{Tr}\left(T^{a} T^{b}\right)=\delta^{a b}$. The coefficients $n_{i}$ are kinematic numerator factors depending on momenta, polarization vectors and spinors. For supersymmetric amplitudes in an on-shell superspace, the numerators will also contain Grassmann parameters. The symmetry factors $S_{i}$ of each graph remove any overcount introduced by summing over all permutations of external legs, as well as any 

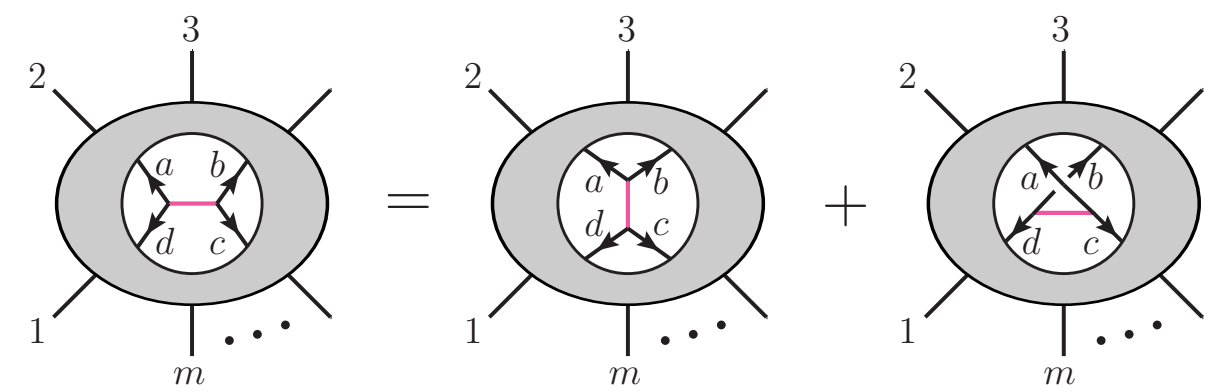

FIG. 1: Pictorial Jacobi relation for a group of three graphs. The graphs can represent color factors or numerator factors. Except for the connections to the central (pink) lines, the graphs are identical in the three cases, as indicated by the common (momentum or color) labels $a, b, c, d$. The gray area represents some unspecified subgraph which is identical in all three graphs.

internal automorphisms - symmetries of a graph with external legs fixed. This form of the amplitude may be obtained from representations involving higher-point contact interactions (as long as they are built out of $\tilde{f}^{a b c}$ 's). One reexpresses all contact terms as the product between a propagator and its inverse; i.e. one inserts $1=p_{\alpha_{i}}^{2} / p_{\alpha_{i}}^{2}$, and assigns the inverse propagator $p_{\alpha_{i}}^{2}$ to be part of a numerator factor $n_{i}$. The duality conjecture [29, 30] states that there should exist a representation of the amplitude where the numerator factors $n_{i}$ satisfy equations in one-to-one correspondence with the Jacobi identities of the color factors. Explicitly, it requires that

$$
C_{i}=C_{j}+C_{k} \quad \Rightarrow \quad n_{i}=n_{j}+n_{k},
$$

where the first relation holds, thanks to the usual color Jacobi identity, for any triplet $(i, j, k)$ of graphs which are identical within the gray region in fig. 1. Moreover, the numerator factors carry the same antisymmetry properties as color factors, i.e. if a color factor changes sign under the interchange of two legs, then so does the corresponding kinematic numerator factor:

$$
C_{i} \rightarrow-C_{i} \quad \Rightarrow \quad n_{i} \rightarrow-n_{i}
$$

These relations are conjectured to hold to all multiplicities, to all loop orders in a weakcoupling expansion, and in any dimension in both supersymmetric and non-supersymmetric Yang-Mills theory. Such relations were noticed long ago for four-point tree amplitudes [42]. Beyond the four-point level, the relations are rather nontrivial and only work after appropriate rearrangements of the amplitudes.

While the sign of the complete numerator factor $C_{i} n_{i}$ of a graph is unambiguous, the sign of each factor, as well as the signs in eq. (2.3), may be changed as a consequence of the relations (2.4) by simply interchanging two lines at any vertex. This ambiguity reflects the different possible sign conventions in Jacobi identities. In this paper, the sign of each color factor $C_{i}$ (and implicitly of $n_{i}$ ) is fixed by the corresponding graph figure: for each integral, $C_{i}$ is built out of the structure constants $\tilde{f}^{a b c}$ corresponding to each trivalent vertex, with legs $a, b, c$ ordered clockwise in the plane of the figure.

The kinematic version of the Jacobi identity (2.3) is the key equation for the duality. At loop level, this equation relates graph numerators at the integrand level, as illustrated in fig. 1. Therefore it is important to properly line up both external and internal momenta. 
There is one such equation for every propagator of every graph. Of course, many of the equations are simply related to each other by automorphic symmetries of graphs, and the fact that any given equation can be obtained starting from each of the three contributing graphs. Simultaneous consideration of all relations gives a system of linear functional equations that the amplitude's numerators should obey. As we will see, only a tiny subset of the possible equations needs to be used when solving the system. Once a tentative solution is found, one must verify that the full set of equations is satisfied, in order to have a duality-satisfying representation of the amplitude. The existence of at least one solution consistent with the unitarity cuts is the critical assumption of the conjecture. Indeed, as we will see, the system of equations at four loops is quite nontrivial and the emergence of a solution is striking.

There is by now substantial evidence in favor of the duality, especially at tree level $(L=$ 0) [43-46], where explicit representations of the numerators in terms of partial amplitudes are known for any number of external legs [47]. A consequence of this duality is the existence of nontrivial relations between the color-ordered partial tree amplitudes of gauge theory [29], which have been proven both from field theory [48] and string theory [49] perspectives. These relations were important in the recent construction of all open-string tree amplitudes [50]. A partial Lagrangian understanding of the duality has also been given [45]. An alternative trace-based presentation of the duality relation (2.3), which emphasizes its group-theoretic structure, was described recently [51].

While less is known at loop level, several nontrivial tests have been carried out. In particular, it has been confirmed that the duality holds for the three-loop four-point amplitude of $\mathcal{N}=4$ sYM theory [30]. (The one- and two-loop four-point amplitudes [8, 52, 53] in this theory also manifestly satisfy the duality). Similarly, the duality-satisfying five-point one-, two- and three-loop amplitudes of $\mathcal{N}=4$ sYM theory have recently been constructed [33]. The color-kinematic duality is also known to hold [30] for the two-loop four-point identicalhelicity amplitude of pure Yang-Mills theory [54]. At present there is no proof that the system of equations generated by the duality (2.3) always has a solution consistent with the unitarity cuts of a given theory, so it needs to be checked case by case. In section III we will find a solution for the four-loop four-point amplitude of $\mathcal{N}=4 \mathrm{sYM}$ theory.

Perhaps more surprising than the duality itself is a consequent relation between gauge and gravity amplitudes. Once the gauge-theory amplitudes are arranged into a form satisfying eq. (2.3), the numerator factors of the corresponding $L$-loop gravity amplitudes, $\mathcal{M}_{m}^{(L)}$, can be obtained simply by multiplying together two copies of gauge-theory numerator factors [29, 30],

$$
\mathcal{M}_{m}^{(L)}=i^{L+1}\left(\frac{\kappa}{2}\right)^{m-2+2 L} \sum_{i \in \Gamma} \int \prod_{l=1}^{L} \frac{d^{D} p_{l}}{(2 \pi)^{D}} \frac{1}{S_{i}} \frac{n_{i} \tilde{n}_{i}}{\prod_{\alpha_{i}} p_{\alpha_{i}}^{2}},
$$

where $\kappa$ is the gravitational coupling. The $\tilde{n}_{i}$ represent numerator factors of a second gaugetheory amplitude and the sum runs over the same set of graphs as in eq. (2.1). At least one family of numerators $\left(n_{i}\right.$ or $\left.\tilde{n}_{i}\right)$ must satisfy the duality (2.3). The construction (2.5) is expected to hold in a large class of gravity theories, including all theories that are the low-energy limits of string theories. At tree level, this double-copy property encodes the KLT relations between gravity and gauge-theory amplitudes [31]. For $\mathcal{N}=8$ supergravity both $n_{i}$ and $\tilde{n}_{i}$ are numerators of $\mathcal{N}=4$ sYM theory.

The double-copy formula (2.5) has been proven [45] for both pure gravity and for $\mathcal{N}=8$ supergravity tree amplitudes, under the assumption that the duality (2.3) holds in the corresponding gauge theories, pure Yang-Mills and $\mathcal{N}=4$ sYM theory, respectively. The non- 
trivial part of the loop-level conjecture is the existence of a representation of gauge-theory amplitudes that satisfies the duality constraints. The double-copy property was explicitly checked for the three-loop four-point amplitude of $\mathcal{N}=8$ supergravity in ref. [30] by comparing eq. (2.5) against a spanning set of unitarity cuts of the previously-calculated amplitude $[9,55]$. Here we perform a similar check for the four-loop four-point amplitude of $\mathcal{N}=8$ supergravity, using the maximal cut method $[41,55]$. The duality and double-copy property have also been confirmed in one- and two-loop five-point amplitudes in $\mathcal{N}=8$ supergravity [33]. For less-than-maximal supergravities, the double-copy property has been checked explicitly for the one-loop four- and five-graviton amplitudes of $\mathcal{N}=4,5,6$ supergravity [56] by showing it matches known results [57]. In the two-loop four-graviton amplitudes in these theories, it has been verified to be consistent with the known infrared divergences and other properties [58]. The double-copy property also leads to some interesting relations between certain $\mathcal{N} \geq 4$ supergravity and subleading-color sYM amplitudes [59].

\section{B. Calculational setup}

We now demonstrate how the conjectured duality between color and kinematics streamlines the construction of integrands of multiloop gauge-theory amplitudes. We first give an overview of the procedure and illustrate it with the three-loop four-point amplitude, before turning to the four-loop case in the following section.

To start the construction we enumerate the graphs with only cubic vertices that can appear in a particular amplitude. Although this step can be carried out in many different ways, we describe one that conveniently also generates the needed duality relations: We assume we have a given set of known cubic graphs (e.g. at four loops we can start with the planar cubic graphs ones given in ref. [5]). Any missing graphs can then be generated by applying the Jacobi relations (2.3) to the set of known graphs. New graphs generated in this way are then added to the list of known ones. This process continues recursively, until no further graphs or relations are found. At the end of the process all cubic graphs related via the duality are known, and all duality relations (2.3) have been written down.

The next step is to solve the relations thus generated. This is the most complicated part of the construction. We can, however, simplify the step by dividing it up into two separate parts, the first of which is straightforward. First use a subset of the duality relations to express all numerator factors in terms of the numerators of a judiciously chosen small set of graphs, which we call "master graphs". We identify master graphs by systematically eliminating numerator factors from the duality relations, via a functional analog of the standard row reduction of systems of linear equations. This problem is analogous to the reduction of loop integrals to a set of master integrals using the Laporta algorithm [60]. In both cases, there is freedom to change the order in which the linear equations are solved. Here, there is a freedom in the choice of master graphs, which is equivalent to a choice of path in solving the system of duality relations. In all cases we have examined, it is convenient to choose the master graphs to be planar (although such a restriction does not necessarily yield the smallest set). This choice has the advantage that the planar contributions are relatively simple and well studied in the literature. In particular, the planar contributions to the four-loop four-point amplitude have a fairly simple form [5]. For the three-loop four-point $\mathcal{N}=4$ sYM amplitude we only need a single master graph [30, 32]. In section III, we will find that at four loops we can express all numerators in terms of the numerators of only two planar master graphs (or a single nonplanar master graph). 
After the reduction of the system of duality constraints, our task is to find explicit expressions for the master numerators. As with any functional equations, a good strategy is to write down Ansätze for the master numerators. The Ansätze are then constrained using input from unitarity cuts, as well as symmetry requirements on both the master numerators and on the numerators derived from them through the duality relations.

In addition to the duality relations (2.3) and unitarity cuts, we may add extra constraints on numerator factors, motivated by our prejudices about the structure and properties of the amplitude. Although not necessary, such constraints, when well chosen, can greatly facilitate the construction. To find the four-loop four-point $\mathcal{N}=4 \mathrm{sYM}$ amplitude we use the following auxiliary constraints, which are known to be valid for the duality-satisfying numerators at three loops [30]:

1. One-loop tadpole, bubble and triangle subgraphs do not appear in any graph.

2. A one-loop $n$-gon subgraph carries no more than $n-4$ powers of loop momentum for that loop.

3. After extracting an overall factor of $s t A_{4}^{\text {tree }}$, the numerators are polynomials in $D$ dimensional Lorentz products of the independent loop and external momenta.

4. Numerators carry the same relabeling symmetries as the graphs.

We will assume that these observations carry over to the four-loop four-point amplitude. If one of these auxiliary conditions had turned out to be too restrictive, it would have led to an inconsistency with either the unitarity cuts or the duality relations. We would then have removed conditions one by one until a consistent solution were found. As we shall see in section III, these auxiliary constraints are quite helpful for quickly finding a dualitysatisfying representation for the four-loop four-point amplitude. A surprisingly small subset of generalized unitarity cuts is then sufficient to completely determine this amplitude.

The specific auxiliary constraints that should be imposed depend on the problem at hand. The third constraint is clearly specific to the four-point amplitude, and should be modified for higher-point amplitudes because they have a more complicated structure. For the fivepoint case, however, a simple generalization has been found [33], involving pre-factors that are proportional [44] to linear combinations of five-point tree-amplitudes. For amplitudes in less supersymmetric theories, the first and second conditions should be relaxed (in addition to the third one), because one-loop triangle and bubble subgraphs are known to appear in such theories.

\section{Three-loop warmup}

To illustrate the above procedure in some detail, we reconstruct the well-studied threeloop four-point amplitude of $\mathcal{N}=4 \mathrm{sYM}$ theory. This amplitude was originally constructed in refs. [9, 55]. A form compatible with the duality (2.3) was then found [30]. Here we describe how to streamline the construction of the latter form, before following a similar procedure at four loops.

A straightforward enumeration shows that there are 17 distinct cubic graphs with three loops and four external legs, which do not have one-loop triangle or bubble (or tadpole) subgraphs. Only 12 contribute to the amplitude, as shown in ref. [30]. These 12 nonvanishing 


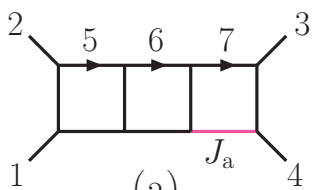

(a)

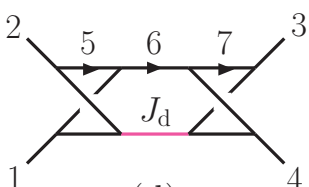

(d)
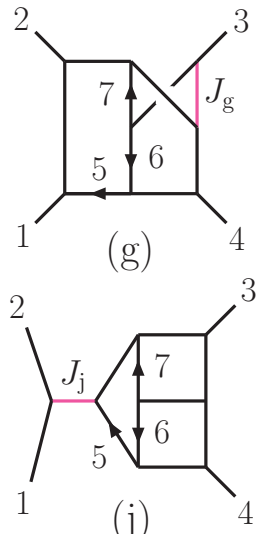

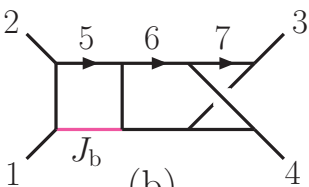

(b)
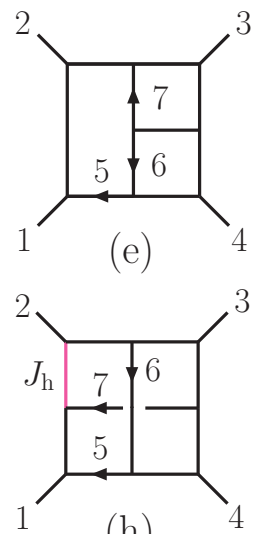

(h)

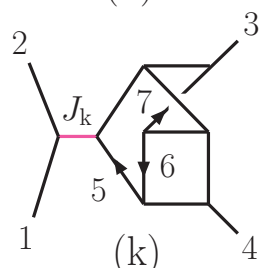

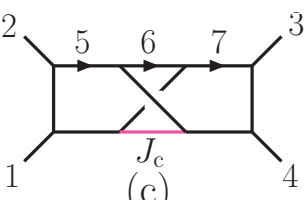

(c)

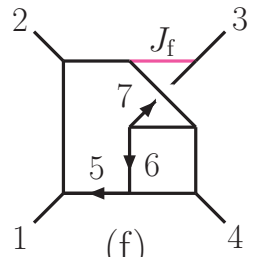

(f)

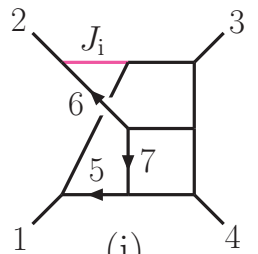

(i)

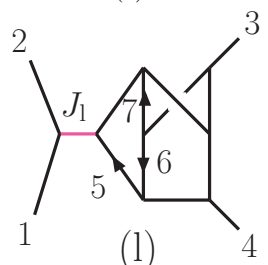

FIG. 2: The 12 nonvanishing graphs used in the construction of the $\mathcal{N}=4$ sYM and $\mathcal{N}=8$ supergravity three-loop four-point amplitude. The shaded (pink) lines mark the application of the duality relation used to determine the numerator of the graph. The external momenta are outgoing and the arrows mark the directions of the labeled loop momenta.

graphs, shown in fig. 2, are sufficient for explaining the construction. (Had we kept all 17 graphs, the construction would be only slightly more involved, with the result that the numerators of the additional five graphs vanish.)

Each numerator depends on three independent external momenta, labeled by $k_{1}, k_{2}, k_{3}$, and on (at most) three independent loop momenta, labeled by $l_{5}, l_{6}, l_{7}$, as well as on the external states. The Mandelstam variables are $s=\left(k_{1}+k_{2}\right)^{2}, t=\left(k_{1}+k_{4}\right)^{2}$ and $u=\left(k_{1}+k_{3}\right)^{2}$. We denote the color-ordered tree-level amplitude by $A_{4}^{\text {tree }} \equiv A_{4}^{\text {tree }}(1,2,3,4)$. The fourpoint amplitudes of $\mathcal{N}=4$ sYM theory are special. Supersymmetry Ward identities fix the external state dependence, and imply that an overall prefactor of $A_{4}^{\text {tree }}$, or equivalently the crossing-symmetric prefactor st $A_{4}^{\text {tree }}[9,55]$, can be extracted from every numerator factor $n^{(x)}$, leaving behind new numerator factors $N^{(x)}$ that depend only on the momenta,

$$
\begin{aligned}
n^{(x)} & =s t A_{4}^{\text {tree }}(1,2,3,4) N^{(x)}, \\
N^{(x)} & \equiv N^{(x)}\left(k_{1}, k_{2}, k_{3}, l_{5}, l_{6}, l_{7}\right) .
\end{aligned}
$$

Here $(x)$ refers to the label for each graph in fig. 2. This result has been argued to be valid in any dimension $D \leq 10$ [11], justifying the third assumption above for these amplitudes. The homogeneity of the Jacobi relations implies that they hold for $N^{(x)}$ just as for $n^{(x)}$. The crossing symmetry of $s t A_{4}^{\text {tree }}$ implies that the symmetry properties of $N^{(x)}$ are the same as those of $n^{(x)}$. 
Next, we write down a subset of the duality relations that allows us to identify the master graphs [32]. For the three-loop four-point amplitude, one such restricted set of duality relations is:

$$
\begin{aligned}
& N^{(\mathrm{a})}=N^{(\mathrm{b})}\left(k_{1}, k_{2}, k_{3}, l_{5}, l_{6}, l_{7}\right), \\
& N^{(\mathrm{b})}=N^{(\mathrm{d})}\left(k_{1}, k_{2}, k_{3}, l_{5}, l_{6}, l_{7}\right), \\
& N^{(\mathrm{c})}=N^{(\mathrm{a})}\left(k_{1}, k_{2}, k_{3}, l_{5}, l_{6}, l_{7}\right), \\
& N^{(\mathrm{d})}=N^{(\mathrm{h})}\left(k_{3}, k_{1}, k_{2}, l_{7}, l_{6}, k_{1,3}-l_{5}+l_{6}-l_{7}\right)+N^{(\mathrm{h})}\left(k_{3}, k_{2}, k_{1}, l_{7}, l_{6}, k_{2,3}+l_{5}-l_{7}\right), \\
& N^{(\mathrm{f})}=N^{(\mathrm{e})}\left(k_{1}, k_{2}, k_{3}, l_{5}, l_{6}, l_{7}\right), \\
& N^{(\mathrm{g})}=N^{(\mathrm{e})}\left(k_{1}, k_{2}, k_{3}, l_{5}, l_{6}, l_{7}\right), \\
& N^{(\mathrm{h})}=-N^{(\mathrm{g})}\left(k_{1}, k_{2}, k_{3}, l_{5}, l_{6}, k_{1,2}-l_{5}-l_{7}\right)-N^{(\mathrm{i})}\left(k_{4}, k_{3}, k_{2}, l_{6}-l_{5}, l_{5}-l_{6}+l_{7}-k_{1,2}, l_{6}\right), \\
& N^{(\mathrm{i})}=N^{(\mathrm{e})}\left(k_{1}, k_{2}, k_{3}, l_{5}, l_{7}, l_{6}\right)-N^{(\mathrm{e})}\left(k_{3}, k_{2}, k_{1},-k_{4}-l_{5}-l_{6},-l_{6}-l_{7}, l_{6}\right), \\
& N^{(\mathrm{j})}=N^{(\mathrm{e})}\left(k_{1}, k_{2}, k_{3}, l_{5}, l_{6}, l_{7}\right)-N^{(\mathrm{e})}\left(k_{2}, k_{1}, k_{3}, l_{5}, l_{6}, l_{7}\right), \\
& N^{(\mathrm{k})}=N^{(\mathrm{f})}\left(k_{1}, k_{2}, k_{3}, l_{5}, l_{6}, l_{7}\right)-N^{(\mathrm{f})}\left(k_{2}, k_{1}, k_{3}, l_{5}, l_{6}, l_{7}\right), \\
& N^{(\mathrm{l})}=N^{(\mathrm{g})}\left(k_{1}, k_{2}, k_{3}, l_{5}, l_{6}, l_{7}\right)-N^{(\mathrm{g})}\left(k_{2}, k_{1}, k_{3}, l_{5}, l_{6}, l_{7}\right),
\end{aligned}
$$

where $k_{i, j} \equiv k_{i}+k_{j}$. For convenience we have suppressed the canonical arguments $\left(k_{1}, k_{2}, k_{3}, l_{5}, l_{6}, l_{7}\right)$ of the numerators on the left-hand side of the equations (2.7), as we will often do in the remainder of the paper. Each relation specifying an $N^{(x)}$ is generated by considering the dual Jacobi relations focusing around the lightly colored (pink) line labeled $J_{x}$ in fig. 2. In general, the duality condition relates triplets of numerators; sometimes, however, one or two of the numerators vanish because the associated graph has a one-loop triangle subgraph forbidden by our auxiliary constraints. Specifically, for five of the above equations, the duality sets pairs of numerators equal; this occurs because the third term in the triplet of numerators of eq. (2.3) vanishes due to the presence of a triangle subgraph. The above system can be used to express any numerator factor in terms of combinations of the numerator $N^{(\mathrm{e})}$, with various different arguments. Thus, graph (e) can be taken as the sole master graph. This is a convenient choice, but not the only possible one; for example, either graph (f) or (g) can also be used as a single master graph. None of the remaining nine graphs, however, can act alone as a master graph.

One valid numerator factor (consistent with unitarity cuts) for graph (e) is the "rung-rule" numerator [53],

$$
N_{\mathrm{rr}}^{(\mathrm{e})}=s\left(l_{5}+k_{4}\right)^{2} .
$$

With this numerator, the graph possesses dual conformal symmetry. However, it turns out that this numerator is incompatible with the duality between color and kinematics (2.3).

We are therefore looking for a modification of $N^{(\mathrm{e})}$ consistent with both the maximal cut of the graph and with the duality constraints (2.7). We start by requiring that the maximal cut of graph (e) is correct, and that the auxiliary constraints in section II B are satisfied. That is, the numerator $N^{(\mathrm{e})}$ has mass dimension four and possesses the symmetry of the graph; no loop momentum for any box subgraph in (e) appears in it (ruling out $l_{6}$ and $l_{7}$ ); and $N^{(\mathrm{e})}$ is at most quadratic in the pentagon loop momenta $l_{5}$. (This last condition is looser than the second auxiliary constraint in section II B; we will tighten it shortly.) The symmetry condition implies that $N^{(\mathrm{e})}$ should be invariant under

$$
\left\{k_{1} \leftrightarrow k_{2}, k_{3} \leftrightarrow k_{4}, l_{5} \rightarrow k_{1}+k_{2}-l_{5}\right\}
$$


The most general polynomial consistent with these constraints is of the form,

$$
N^{(\mathrm{e})}=s\left(l_{5}+k_{4}\right)^{2}+(\alpha s+\beta t) l_{5}^{2}+(\gamma s+\delta t)\left(l_{5}-k_{1}\right)^{2}+(\alpha s+\beta t)\left(l_{5}-k_{1}-k_{2}\right)^{2},
$$

where the four parameters $\alpha, \beta, \gamma, \delta$ are to be determined by further constraints. All added terms are proportional to inverse propagators and therefore vanish on the maximal cut. Thus, since eq. (2.8) is consistent with the maximal cuts so is eq. (2.10).

According to the second auxiliary constraint in section II B, the numerator of a pentagon subgraph should be at most linear in the corresponding loop momentum, not quadratic as assumed above. Therefore the coefficient of $l_{5}^{2}$ in eq. (2.10) should vanish, yielding the relations $\gamma=-1-2 \alpha$ and $\delta=-2 \beta$. Consequently, the Ansatz for $N^{(\mathrm{e})}$ is reduced to

$$
N^{(\mathrm{e})}=s\left(\tau_{45}+\tau_{15}\right)+(\alpha s+\beta t)\left(s+\tau_{15}-\tau_{25}\right),
$$

where we use the notation,

$$
\tau_{i j} \equiv 2 k_{i} \cdot l_{j}(i \leq 4, j \geq 5), \quad \tau_{i j} \equiv 2 l_{i} \cdot l_{j}(i, j \geq 5) .
$$

Now there are just two undetermined parameters, $\alpha$ and $\beta$.

To determine one of the remaining parameters we use the properties of graph $(\mathrm{j})$, and the expression for its numerator in terms of the numerator of graph (e), which is given by the 9 th duality constraint in eq. (2.7). Inserting eq. (2.11) into this relation leads to

$$
N^{(\mathrm{j})}=s(1+2 \alpha-\beta)\left(\tau_{15}-\tau_{25}\right)+\beta s(t-u) .
$$

Because the smallest loop in graph $(\mathrm{j})$ carrying $l_{5}$ is a box subgraph, our auxiliary constraints require that this momentum be absent from $N^{(\mathrm{e})}$. Setting the first term in eq. (2.13) to zero implies that $\beta=1+2 \alpha$, which in turn leads to

$$
\begin{aligned}
& N^{(\mathrm{e})}=s\left(\tau_{45}+\tau_{15}\right)+(\alpha(t-u)+t)\left(s+\tau_{15}-\tau_{25}\right), \\
& N^{(\mathrm{j})}=(1+2 \alpha)(t-u) s,
\end{aligned}
$$

leaving undetermined a single parameter $\alpha$.

There are a variety of ways to determine the final parameter. For example, one can use planar cuts to enforce that the planar part of the amplitude is correctly reproduced. A particularly instructive method is to use the duality relations to obtain the numerator for planar graph (a) in terms of master numerator $N^{(\mathrm{e})}$. The numerator $N^{(\mathrm{a})}$ is quite simple once we impose the condition that a one-loop box subgraph cannot carry loop momentum. Since three independent one-loop subgraphs of graph (a) are boxes, the numerator $N^{(a)}$ cannot depend on any loop momenta. Indeed, the iterated two-particle cuts, or equivalently the rung insertion rule [53], immediately fix this contribution to be

$$
N^{(\mathrm{a})}=s^{2}
$$

Solving the duality relations (2.7) to express $N^{(\mathrm{a})}$ in terms of $N^{(\mathrm{e})}$ we find,

$$
\begin{aligned}
N^{(\mathrm{a})}= & N^{(\mathrm{e})}\left(k_{1}, k_{2}, k_{4},-k_{3}+l_{5}-l_{6}+l_{7}, l_{5}-l_{6},-l_{5}\right) \\
& +N^{(\mathrm{e})}\left(k_{2}, k_{1}, k_{4},-k_{3}-l_{5}+l_{7},-l_{5}, l_{5}-l_{6}\right) \\
& -N^{(\mathrm{e})}\left(k_{4}, k_{1}, k_{2}, l_{6}-l_{7}, l_{6}, l_{5}-l_{6}\right)-N^{(\mathrm{e})}\left(k_{4}, k_{2}, k_{1}, l_{6}-l_{7}, l_{6},-l_{5}\right) \\
& -N^{(\mathrm{e})}\left(k_{3}, k_{1}, k_{2}, l_{7}, l_{6}, l_{5}-l_{6}\right)-N^{(\mathrm{e})}\left(k_{3}, k_{2}, k_{1}, l_{7}, l_{6},-l_{5}\right) .
\end{aligned}
$$


Plugging in the value of the numerator factor $N^{(\mathrm{e})}$ in eq. (2.14), we obtain

$$
N^{(\mathrm{a})}=s^{2}+(1+3 \alpha)\left(\left(\tau_{16}-\tau_{46}\right) s-2\left(\tau_{17}+\tau_{37}\right) s+\left(\tau_{16}-2 \tau_{17}-\tau_{26}+2 \tau_{27}\right) t+4 u t\right) .
$$

Demanding that this expression matches the numerator factor $N^{(a)}$ given in eq. (2.16), or alternatively that it is independent of loop momenta, fixes the final parameter to be $\alpha=-1 / 3$. This constraint completely determines the numerator of graph (e) to be

$$
N^{(\mathrm{e})}=s\left(\tau_{45}+\tau_{15}\right)+\frac{1}{3}(t-s)\left(s+\tau_{15}-\tau_{25}\right),
$$

matching the result of ref. [30].

Remarkably, numerator (e) in eq. (2.19) generates all other numerators $N^{(x)}$, via eq. (2.7), giving us the entire integrand at three loops. For all graphs, the resulting numerators reproduce the expressions quoted in ref. [30], and the resulting amplitude matches previous expressions $[9,55]$ on all $D$-dimensional unitarity cuts. As already noted, it is highly nontrivial to have a consistent solution where all duality relations hold, all numerators have the graph symmetries and all unitarity cuts are correct. Squaring these numerators $N^{(x)}$, using eq. (2.5), immediately yields the numerators for the three-loop four-point $\mathcal{N}=8$ supergravity amplitude. This form has also been confirmed against previous expressions $[9,55]$ on a spanning set of $D$-dimensional unitarity cuts [30].

We shall use the same streamlined strategy to construct the four-loop four-point amplitude in section III. Before carrying out this construction, however, we need to address an important subtlety that appears in the construction of the three-loop amplitude and affects the four-loop construction as well.

\section{Comment on one-particle-reducible graphs and snails}

Beyond tree-level, the on-shell three-point amplitudes of $\mathcal{N}=4 \mathrm{sYM}$ theory vanish. The appearance of one-particle reducible (1PR) graphs in the three-loop four-point amplitude may therefore seem surprising. Indeed, graphs (i), (j) and (k) of fig. 2 do not appear in the original representations of the same amplitude $[9,55]$. The existence of $1 \mathrm{PR}$ graphs may seem to imply that the three-point amplitude is non-vanishing. However, these graphs' numerators are proportional to the Mandelstam invariant $s$, which is also the inverse propagator for the sole line on which the graph is $1 \mathrm{PR}$. Thus, the superficially $1 \mathrm{PR}$ graphs are in fact just oneparticle-irreducible (1PI) contact graphs. Even though they are kinematically equivalent to 1PI graphs, the non-contact form of graphs (i), (j) and (k) in fig. 2 is needed to describe easily their color structure, and to allow the amplitude to obey the duality (2.3) between color and kinematics. As we shall see, this feature continues at four loops, where we encounter, not only graphs with three-point subgraphs, but also nontrivial two-point subgraphs. Some of these graphs contain four-loop two-point bubble subgraphs on external legs, and must be treated with particular care.

At first sight, it may appear surprising that two- and three-point subgraphs show up; indeed in $\mathcal{N}=4$ sYM theory we expect the vanishing of on-shell two- and three-point loop amplitudes. This property has been known in string theory for some time [61]. By taking the low-energy limit, it should hold in field theory as well. A direct field theory argument for the vanishing of the on-shell two-point function can be made as follows: Quite generally, 
a (diagonal) on-shell two-point loop contribution represents a correction to the mass of the corresponding field. Gauge invariance forbids such a term from being generated in the gluon two-point function. The chirality of $\mathcal{N}=4 \mathrm{sYM}$ gluinos forbids such mass terms from being generated by perturbative quantum effects for fermions as well. Thus, gluon and gluino two-point functions vanish on shell. Manifest off-shell $\mathcal{N}=1$ supersymmetry, which can be maintained, then implies that the scalar field two-point function also vanishes on shell.

We can also argue that three-point amplitudes vanish on shell. Because $\mathcal{N}=4$ supersymmetry relates all such amplitudes to each other, it suffices to focus on the scattering amplitude of two fermions and one scalar. Up to an $S U(4) R$-symmetry transformation, we may further assume that neither of the fermions is the $\mathcal{N}=1$ superpartner of the gluon. Thus we consider only the interaction between $\mathcal{N}=1$ matter multiplets. Conservation of the matter $R$-symmetry subgroup $S U(3)$ requires that the three-field interaction is controlled by $S U(3)$ invariance, and thus is either holomorphic or antiholomorphic. Now, in the effective action language, the three-point amplitude originates either from terms in the superpotential or the Kähler potential. Due to the perturbative nonrenormalization of the superpotential [62], only the tree amplitude comes from the former. A nonvanishing loop amplitude can only originate from a correction to the Kähler potential. For this case, a nonvanishing full superspace integral and Lorentz invariance require that the product of three chiral superfields containing the relevant wave functions must be accompanied by at least two additional superderivatives. In turn, this implies that the product of one scalar and two fermion wave functions is always accompanied by an external momentum invariant, originating from the superspace integration measure. For massless fields, any such product vanishes on shell. Thus, all quantum corrections to three-point amplitudes in $\mathcal{N}=4 \mathrm{sYM}$ theory vanish on shell, completing the argument.

While these arguments confirm the vanishing of two- and three-point amplitudes at oneloop and beyond, we emphasize that this does not mean that we cannot have graphs with two- and three-point subgraphs. However, when such graphs appear they should always carry factors that make their contributions vanish whenever legs are cut (placed on shell) to isolate two- and three- point subamplitudes. Indeed, we shall find that at four points, through four loops, all such graphs with three- or four-point subgraphs can be absorbed as contact terms in other graphs. This property is consistent with the fact that previous representations of the three- and four-loop amplitudes $[9,11,55]$ do not use any $1 \mathrm{PR}$ graphs with two- or three-point loop subgraphs.

At $L$ loops, the four-point amplitude in $\mathcal{N}=4$ sYM theory is expected to have a representation with at most $2(L-2)$ powers of the loop momentum in the numerator of each $1 \mathrm{PI}$ cubic graph $[8,63]$. At three loops, this power counting allows for cancellation of one internal propagator, as in graphs (i), (j) and (k) of fig. 2. However, it precludes the existence of two-point graphs or propagator corrections (and tadpole graphs), which would require two inverse propagators or four powers of the loop momentum in the numerator. On the other hand, at four loops and beyond, such graphs can and indeed do appear. Propagator corrections can be of two types:

1. on internal legs, as shown in fig. 3(a), or

2. on external legs, as shown in fig. 3(c).

In both cases the graph's numerators must contain momentum invariants that cancel out the unwanted poles, so that they are kinematically equivalent to the 1PI graphs shown in fig. 3(b) and fig. 3(d), respectively. 


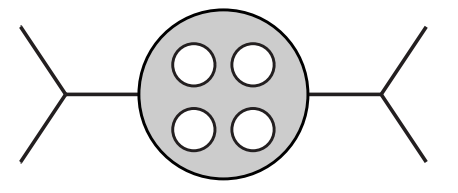

(a)

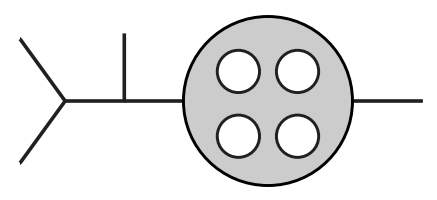

(c)
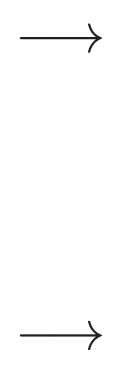

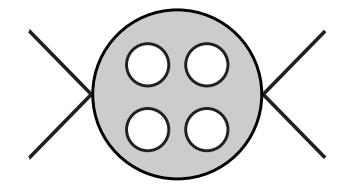

(b)

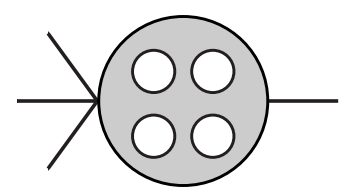

(d)

FIG. 3: Graphs (a) and (c) are generic propagator correction graphs that can appear at four loops and beyond if we have a cubic organization of graphs. Graphs (b) and (d) are rewritings of these graphs, which make explicit that in $\mathcal{N}=4 \mathrm{sYM}$ theory numerator factors always cancel the propagators that are external to the loops in the four-point amplitude.

For case 1, this cancellation is straightforward because the momentum invariant is nonvanishing for generic on-shell kinematics. For case 2, the external leg corrections, the mechanism is more subtle. On the one hand, because the amplitudes have on-shell external legs, a propagator in fig. 3(c) diverges: $1 /\left(k_{1}+k_{2}+k_{3}\right)^{2}=1 / 0$. On the other hand, from the vanishing of the on-shell two-point function we expect that the numerator of fig. 3(c) is proportional to $k_{4}^{2}=0$. In order to resolve this $0 / 0$ ambiguity, we need to regulate the external leg by taking $k_{4}^{2} \neq 0$, and cancel factors of $k_{4}^{2}$ between numerator and denominator. This procedure yields the "snail graph" ${ }^{1}$ in fig. $3(\mathrm{~d})$, which is perfectly well behaved at the level of the integrand, even with all external momenta on-shell.

It is important to note that the snail graph in fig. 3(d) contains a scale-free integral, which vanishes by the usual rules of dimensional regularization. We cannot, however, simply ignore these contributions. In dimensional regularization, scale-free integrals evaluate to zero because of cancellations between infrared and UV singularities. Ignoring the snail graph contributions in $\mathcal{N}=4$ sYM theory would lead to incorrect values for the UV divergences. ${ }^{2}$ Since we are interested in this paper in the coefficient of the UV divergences, these snail graphs must be included.

While enforcing the duality constraints (2.3) brings the phenomenon of snail graphs to the forefront, we emphasize that the potential appearance of such contributions to amplitudes is independent of the color-kinematic duality. Snail contributions can in principle occur within any representation; it is therefore important to always check the unitarity cuts for such contributions. Because snails are associated with external leg contributions, ordinary unitarity cuts fail to detect them, and generalized cuts are required. The momenta of the states crossing the cut must either be complex, or else have an indefinite sign of their energy.

\footnotetext{
${ }^{1}$ With suitable imagination, the graph resembles a snail (as much as a penguin diagram resembles a penguin).

${ }^{2}$ In QCD, propagator corrections on external legs can be ignored because the UV divergences are known a priori. It is therefore quite simple to restore the missing terms. In $\mathcal{N}=4 \mathrm{sYM}$ theory, UV divergences in $D>4$ are unknown a priori.
} 
(We have examined such cuts, and have confirmed thereby that no snail contributions are present in the representation found in ref. [11].)

Although the snail contributions are important for $\mathcal{N}=4 \mathrm{sYM}$ amplitudes, they will not infect the corresponding $\mathcal{N}=8$ supergravity amplitudes. This may be understood heuristically as a consequence of the double-copy formula (2.5). In $\mathcal{N}=4 \mathrm{sYM}$ theory, graphs of the form in fig. 3(c) carry a factor of 0 in their numerator to cancel the $1 / 0$ from the propagator. In $\mathcal{N}=8$ supergravity we get a second factor of 0 from the second copy, making the numerator vanish faster than the denominator, and giving a vanishing snail contribution. Below we confirm this heuristic argument directly from unitarity cuts.

Finally, we remark that very similar considerations appear in the analysis of inverse derivative factors arising from the collision of vertex operators in the discussion of nonrenormalization conditions for amplitudes in superstring theory - see section 3.2 of ref. [64].

\section{THE FOUR-LOOP FOUR-POINT INTEGRAND}

We now turn to the construction of the four-loop four-point amplitude and follow the same strategy as described in the previous section for the corresponding three-loop amplitude.

\section{A. Overview of the result}

We will find that, in terms of the 85 distinct graphs of figs. 5-11, the four-loop sYM amplitude is given by

$$
\mathcal{A}_{4}^{(4)}=g^{10} s t A_{4}^{\text {tree }} \sum_{\mathcal{S}_{4}} \sum_{i=1}^{85} \int\left(\prod_{j=5}^{8} \frac{d^{D} l_{j}}{(2 \pi)^{D}}\right) \frac{1}{S_{i}} \frac{N_{i}\left(k_{j}, l_{j}\right) C_{i}}{\prod_{\alpha_{i}=1}^{13} p_{\alpha_{i}}^{2}},
$$

where $l_{5}, l_{6}, l_{7}, l_{8}$ are the four independent loop momenta and $k_{1}, k_{2}, k_{3}$ are the three independent external momenta. The $p_{\alpha_{i}}$ are the momenta of the internal propagators (corresponding to the internal lines of each graph $i$ ), and are linear combinations of the independent loop momenta $l_{j}$ and the external momenta $k_{m}$. In the case of $1 \mathrm{PR}$ graphs, some $p_{\alpha_{i}}$ will depend only on the external momenta. As usual, $d^{D} l_{j} /(2 \pi)^{D}$ is the $D$-dimensional integration measure for the $j^{\text {th }}$ loop momentum. The numerator factors $N_{i}\left(k_{j}, l_{j}\right)$ are polynomial in both internal and external momenta, and are given in appendix B. The color factors $C_{i} \equiv C_{i}^{a_{1} a_{2} a_{3} a_{4}}$ are collected online [38], but they can also be read directly off the figures. The full amplitude is obtained by summing over the group $\mathcal{S}_{4}$ of 24 permutations of the external leg labels. Overcounts are removed by the symmetry factors $S_{i}$, which include both external symmetry factors (related to the overcount from the sum over $\mathcal{S}_{4}$ ), as well as any internal symmetry factors associated with automorphisms of the graphs holding the external legs fixed. As at three loops, we extract the crossing-symmetric, $\mathcal{S}_{4}$-invariant prefactor $s t A_{4}^{\text {tree }}$, which contains all dependence on the external states. (Notice that we have used a slightly different notation for the independent loop momenta $l_{j}$ in eq. (3.1), compared with $p_{l}$ in eq. (2.1).)

Out of the 85 integrals in eq. (3.1), graphs 50 and 79 are somewhat peculiar: Their integrands are nonvanishing, but they integrate to zero. The vanishing of their integrals can be seen from symmetry considerations alone. For example, graph 50 has a symmetry exchanging legs 1 and 4, and legs 2 and 3, flipping the graph across a vertical midline. It is easy to check that the color graph $C_{50}$ picks up a minus sign under this operation; therefore 
the kinematic integrand must also be antisymmetric, causing the integral to vanish. In fact, as the duality between color and kinematics might suggest, the color factors $C_{50}$ and $C_{79}$ vanish after the internal color sum is carried out (for any gauge group $G$ ). However, both graphs give nonvanishing contributions to the $\mathcal{N}=8$ supergravity amplitude. Therefore we retain them here. (While the vanishing gauge-theory integrals are odd under the above relabeling of the loop momenta, the double-copy property makes the gravity integrals even under the same relabeling.)

As we discussed in section IID, graphs 83-85, (displayed in fig. 11), superficially appear as propagator corrections on external legs. These graphs give rise to the snail contributions described there, after an external propagator is canceled by a corresponding factor in the numerator.

Using the double-copy relation (2.5), the four-loop four-point $\mathcal{N}=8$ supergravity amplitude is obtained simply by trading the color factor $C_{i}$ for $\tilde{n}_{i}=s t \tilde{A}_{4}^{\text {tree }} N_{i}$ in eq. (3.1). Employing the relation $s^{2} t^{2} A_{4}^{\text {tree }} \tilde{A}_{4}^{\text {tree }}=i s t u M_{4}^{\text {tree }}$ and changing the gauge coupling to the gravitational coupling, we have

$$
\mathcal{M}_{4}^{(4)}=-\left(\frac{\kappa}{2}\right)^{10} \text { stu } M_{4}^{\text {tree }} \sum_{\mathcal{S}_{4}} \sum_{i=1}^{82} \int\left(\prod_{j=5}^{8} \frac{d^{D} l_{j}}{(2 \pi)^{D}}\right) \frac{1}{S_{i}} \frac{N_{i}^{2}\left(k_{j}, l_{j}\right)}{\prod_{\alpha_{i}=1}^{13} p_{\alpha_{i}}^{2}}
$$

where $N_{i}\left(k_{j}, l_{j}\right)$ are the gauge-theory numerator factors given in appendix B. In contrast to the sYM amplitudes, potential snail contributions from graphs 83-85 vanish identically, as expected from our heuristic argument in section IID, and confirmed by an analysis of the unitarity cuts.

The symmetry factors appearing in eqs. (3.1) and (3.2) are given explicitly as:

$$
\begin{aligned}
\sum_{i=1}^{85} \frac{1}{S_{i}} I_{i} & =\frac{1}{4} I_{1}+\frac{1}{4} I_{2}+\frac{1}{16} I_{3}+\frac{1}{4} I_{4}+\frac{1}{8} I_{5}+\frac{1}{2} I_{6}+\frac{1}{2} I_{7}+I_{8}+\frac{1}{4} I_{9}+\frac{1}{4} I_{10}+\frac{1}{2} I_{11}+\frac{1}{4} I_{12}+\frac{1}{2} I_{13} \\
& +\frac{1}{2} I_{14}+\frac{1}{4} I_{15}+I_{16}+\frac{1}{2} I_{17}+I_{18}+I_{19}+I_{20}+I_{21}+I_{22}+I_{23}+\frac{1}{2} I_{24}+I_{25}+I_{26} \\
& +\frac{1}{2} I_{27}+\frac{1}{4} I_{28}+I_{29}+\frac{1}{2} I_{30}+\frac{1}{2} I_{31}+I_{32}+I_{33}+\frac{1}{2} I_{34}+I_{35}+I_{36}+\frac{1}{2} I_{37}+\frac{1}{4} I_{38} \\
& +\frac{1}{2} I_{39}+\frac{1}{4} I_{40}+\frac{1}{2} I_{41}+I_{42}+I_{43}+\frac{1}{2} I_{44}+\frac{1}{4} I_{45}+\frac{1}{2} I_{46}+\frac{1}{8} I_{47}+\frac{1}{2} I_{48}+\frac{1}{2} I_{49}+\frac{1}{8} I_{50} \\
& +\frac{1}{2} I_{51}+I_{52}+\frac{1}{4} I_{53}+\frac{1}{2} I_{54}+\frac{1}{2} I_{55}+\frac{1}{2} I_{56}+\frac{1}{2} I_{57}+\frac{1}{2} I_{58}+\frac{1}{2} I_{59}+\frac{1}{2} I_{60}+\frac{1}{4} I_{61}+\frac{1}{2} I_{62} \\
& +\frac{1}{2} I_{63}+\frac{1}{2} I_{64}+\frac{1}{2} I_{65}+\frac{1}{4} I_{66}+\frac{1}{2} I_{67}+\frac{1}{4} I_{68}+\frac{1}{4} I_{69}+\frac{1}{2} I_{70}+\frac{1}{8} I_{71}+\frac{1}{2} I_{72}+\frac{1}{2} I_{73}+\frac{1}{4} I_{74} \\
& +\frac{1}{4} I_{75}+\frac{1}{2} I_{76}+\frac{1}{2} I_{77}+\frac{1}{4} I_{78}+\frac{1}{8} I_{79}+\frac{1}{16} I_{80}+\frac{1}{8} I_{81}+\frac{1}{16} I_{82}+\frac{1}{4} I_{83}+\frac{1}{2} I_{84}+\frac{1}{4} I_{85},
\end{aligned}
$$

where $I_{i}$ should be interpreted only as placeholders for the graphs, including both the numerator or color factors, in either theory.

\section{B. The calculation}

As at three loops, the construction of the amplitude begins by writing down a sufficient number of duality constraints so that a set of master numerators can be identified. We

have constructed a set of duality equations similar to the three-loop ones of eq. (2.7). In appendix A we collect a set of simplified equations derived from these duality constraints 


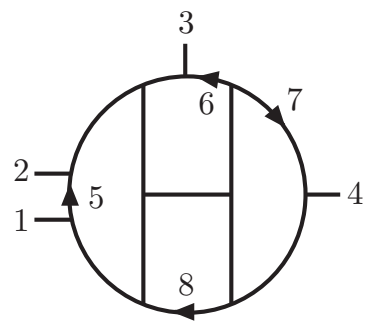

(18)

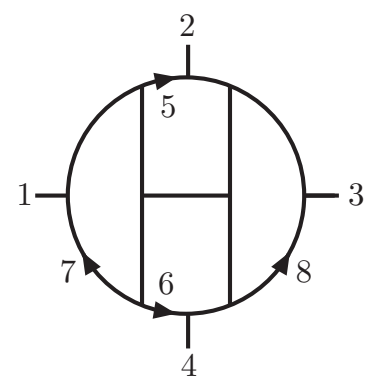

(28)

FIG. 4: The planar master graphs, 18 and 28. The numerators and color factors of all other graphs are generated from the numerators and color factors of these two graphs through kinematic Jacobi relations.

by imposing the four auxiliary constraints presented in section II B. Because of these additional simplifications, the duality equations in the appendix are valid only for $\mathcal{N}=4 \mathrm{sYM}$ theory. The duality equations allow us to express all non-snail numerators directly as linear combinations of the numerators $N_{18}$ and $N_{28}$. The corresponding graphs are shown in fig. 4; we will choose them as the master graphs.

It is interesting to note that as an alternative we can use a single nonplanar master graph that does the same job, such as graph 33 (or equivalently 35 or 36, which have identical numerators up to a sign). However, we prefer to use planar graphs as master graphs because their numerators have a somewhat simpler structure. If we choose planar graphs as master graphs then the minimal number is two. In our treatment the snail contributions are only given partially in terms of the master numerators, because the latter are specified using on-shell external kinematics, whereas the numerators of the former require an off-shell regularization to be nonvanishing.

Our next task is to determine the master numerators. To this end we begin by constructing an Ansatz for the numerator factors $N_{18}$ and $N_{28}$ that satisfies the auxiliary constraints discussed in section IIB and a restricted set of duality relations. We then constrain the Ansatz by demanding that other duality relations are satisfied, and that the numerator factors of the other integrals obey the auxiliary constraints. For both graphs, the numerator must be independent of loop momenta $l_{7}$ and $l_{8}$ because they are assigned to one-loop box subgraphs, whose momenta should not appear in their numerators. Similarly, momenta $l_{5}$ and $l_{6}$ are assigned to one-loop pentagon subgraphs, so $N_{18}$ and $N_{28}$ should be no more than linear in these momenta, according to our auxiliary constraints. Thus, each of the two master numerators should be a polynomial built from the monomials,

$$
M=\left\{s^{3}, s t^{2}, s^{2} t, t^{3}, \tau_{i 5} s^{2}, \tau_{i 5} t^{2}, \tau_{i 5} s t, \tau_{i 6} s^{2}, \tau_{i 6} t^{2}, \tau_{i 6} s t, \tau_{i 5} \tau_{j 6} s, \tau_{i 5} \tau_{j 6} t, \tau_{56} s^{2}, \tau_{56} t^{2}, \tau_{56} s t\right\},
$$

where $i=1,2,3$ labels the three independent external momenta, $k_{1}, k_{2}, k_{3}$. In total this gives us a polynomial with 43 terms for each master graph. Labeling the monomials consecutively as $M_{j}$, and including arbitrary coefficients, we have as our starting Ansatz,

$$
N_{18}=\sum_{j=1}^{43} a_{j} M_{j}, \quad N_{28}=\sum_{j=1}^{43} b_{j} M_{j} .
$$

The 86 free coefficients $a_{j}$ and $b_{j}$ are to be determined from various consistency conditions obtained from the color-kinematic duality, graph symmetries and unitarity cuts. The number 


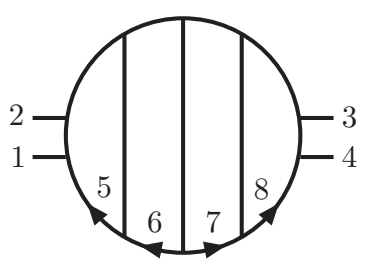

(1)

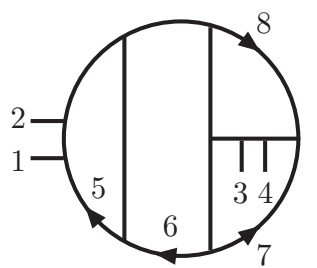

(4)

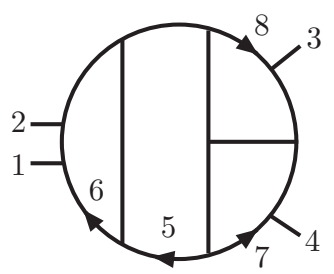

(6)

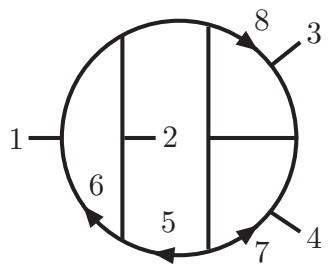

(9)

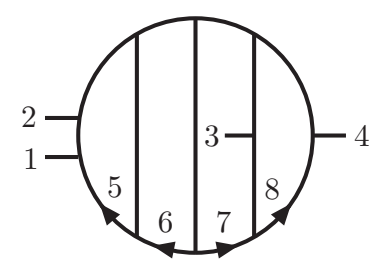

(2)

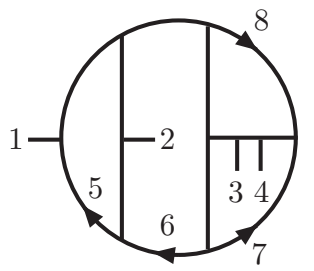

(5)

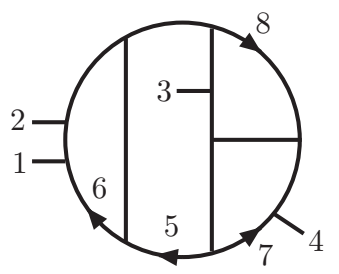

(7)

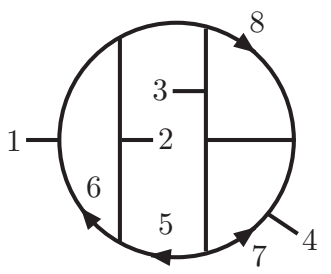

(10)

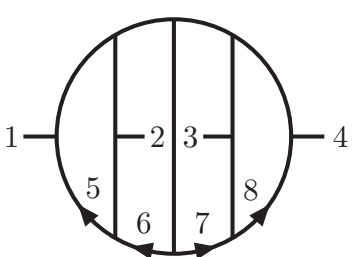

(3)

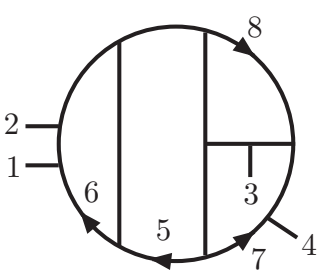

(8)

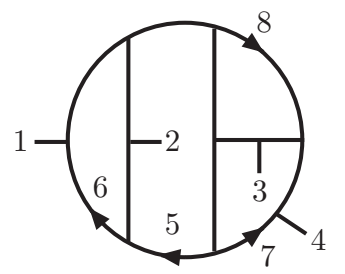

(11)

FIG. 5: Cubic graphs 1 to 11 that contribute to the four-loop four-point amplitude of $\mathcal{N}=4 \mathrm{sYM}$ theory and $\mathcal{N}=8$ supergravity. The labels 1 to 4 , indicate the legs carrying external momenta $k_{1}$ to $k_{4}$. The labels 5 to 8 indicate the propagators carrying the independent loop momenta $l_{5}$ to $l_{8}$. The arrows indicate the direction of the momentum. The graphs also specify the color factor of the graph, simply by dressing each cubic vertex with an $\tilde{f}^{a b c}$, respecting the clockwise ordering of lines at each vertex.

of free parameters that need to be determined in this construction is remarkably small, considering the expected analytic complexity of amplitudes at four loops.

Using the 86-parameter Ansatz and the solution to the restricted set of duality constraints listed in appendix A gives us expressions for the numerator factors of any of the 82 nonsnail graphs appearing in the amplitude. (The snail graphs will be determined below in terms of the non-snail graphs using generalized unitarity cuts.) These expressions do not yet satisfy all duality constraints; thus far we have imposed only the relatively few relations in appendix A sufficient to determine all numerators in terms of the master numerators, but we have not yet accounted for the complete set of duality relations. To further constrain the master Ansatz we could require that all other dual Jacobi relations are satisfied; there are on the order of $13 \times 85$ such functional relations (not all independent). An alternate strategy, which we follow here, is to first impose the consistency constraints on the numerators of the 


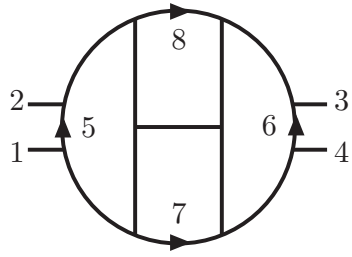

(12)

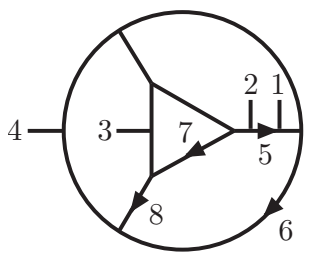

(15)

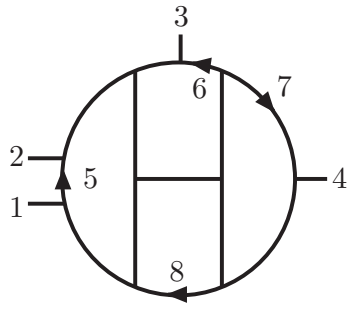

(18)

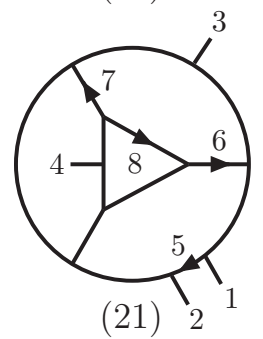

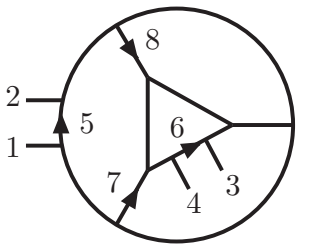

(13)

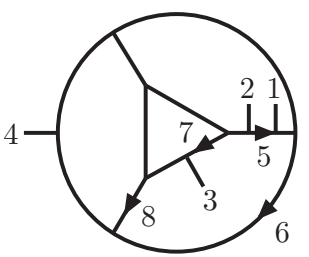

(16)

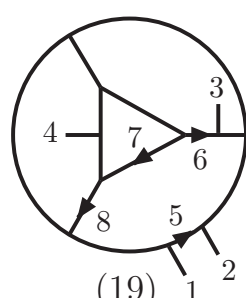

(19)

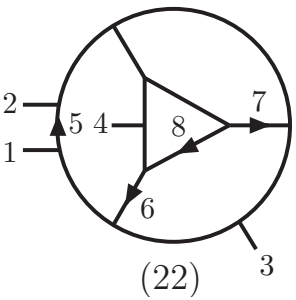

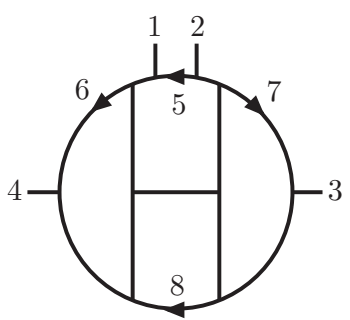

(14)

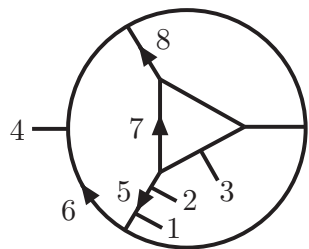

(17)

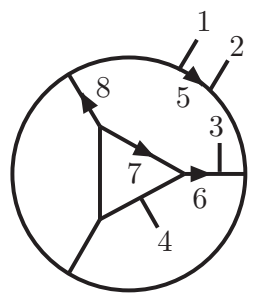

(20)

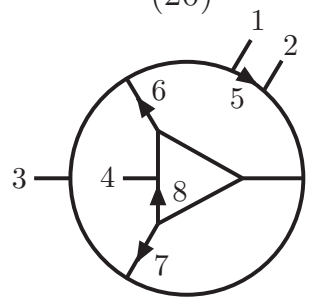

(23)

FIG. 6: Cubic graphs 12 to 23 that contribute to the four-loop four-point amplitude of $\mathcal{N}=4 \mathrm{sYM}$ theory and $\mathcal{N}=8$ supergravity. The labeling is the same as for fig. 5 .

graphs derived from $N_{18}$ and $N_{28}$ through eqs. (A4) and (A5). After obtaining a complete solution for all 86 parameters appearing in the ansatz, we then verify that they indeed satisfy all remaining duality relations and unitarity cuts. An advantage of this strategy is that it allows us to illustrate the remarkably small number of unitarity cuts needed to find the complete amplitude, including nonplanar contributions.

As we shall see, to construct the complete amplitude we need only information about the unitarity cuts of the four-loop planar amplitude, obtained previously in refs. [5, 11]. The list of constraints needed to fix all 86 parameters in the Ansatz, thus determining the amplitude, is remarkably short. It is sufficient to enforce:

1. the graph automorphism symmetries on numerators $N_{12}, N_{14}$ and $N_{28}$;

2. the maximal cut of graph 12 ;

3. the next-to-maximal cut of graph 14, where $l_{5}$ is the off-shell leg. Graph 68 also contributes to this cut. 


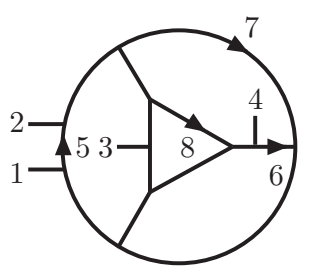

(24)

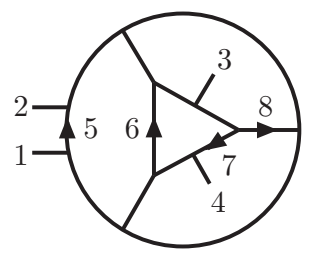

(27)

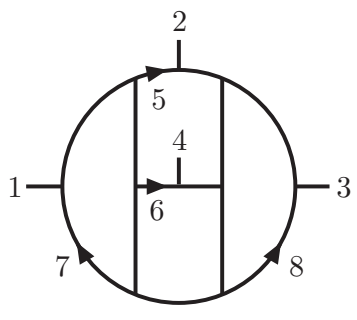

(30)
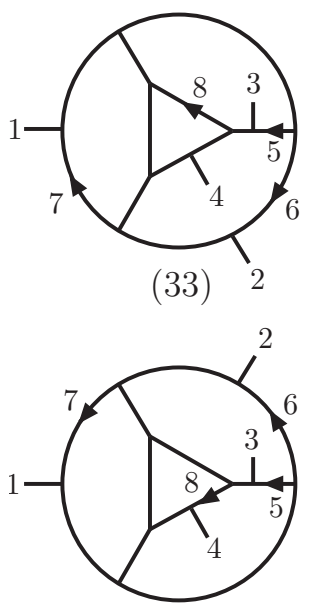

(36)

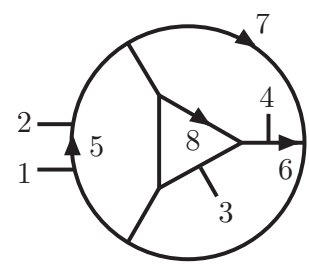

(25)

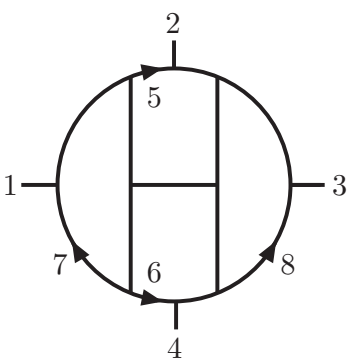

(28)

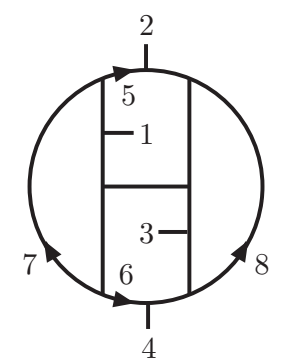

(31)

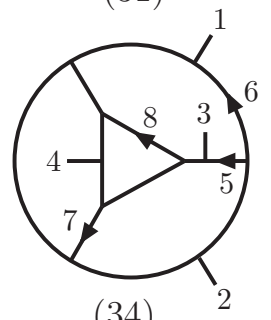

(34)

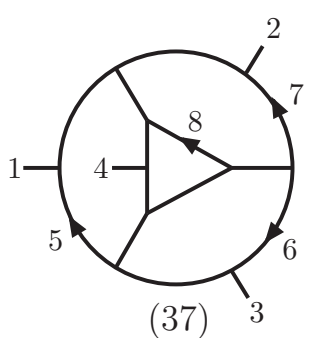

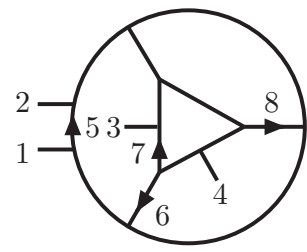

(26)

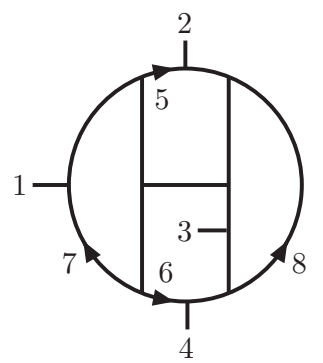

(29)

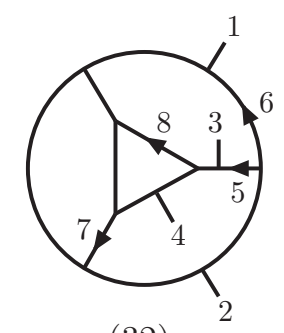

(32)
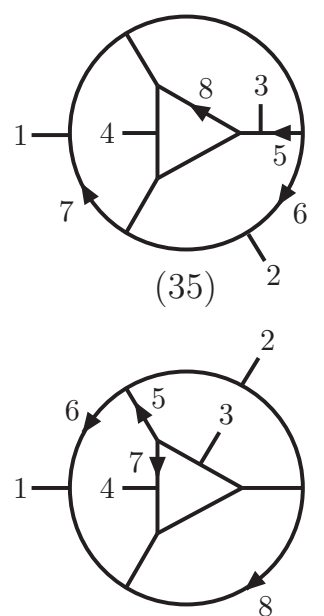

(38)

FIG. 7: Cubic graphs 24 to 38 that contribute to the four-loop four-point amplitude of $\mathcal{N}=4$ sYM theory and $\mathcal{N}=8$ supergravity. The labeling is the same as for fig. 5 .

Strikingly, only two rather simple planar cuts are needed to fully determine the amplitude. Let us now discuss some details of fixing the parameters.

We start by analyzing the consequences of the symmetries of the master graph 28: This graph is invariant under two independent transformations:

$$
\left\{k_{1} \leftrightarrow k_{3}, l_{5} \rightarrow k_{2}-l_{5}, l_{6} \rightarrow k_{4}-l_{6}, l_{7} \leftrightarrow l_{8}\right\}
$$




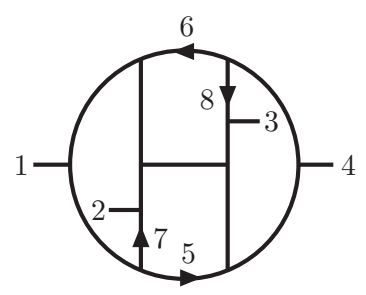

(39)

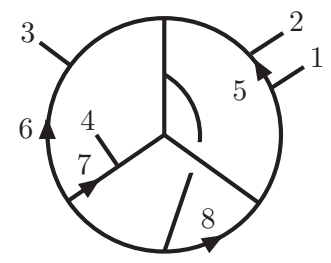

(42)

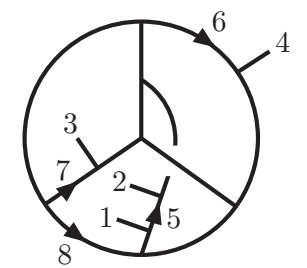

(45)

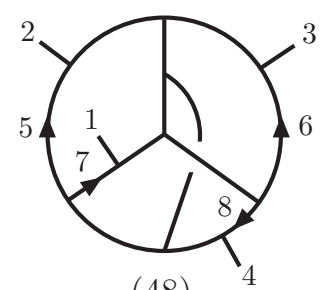

(48)

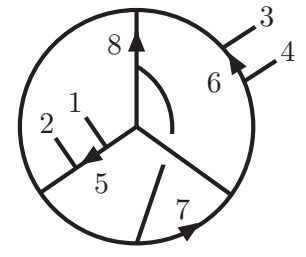

(40)
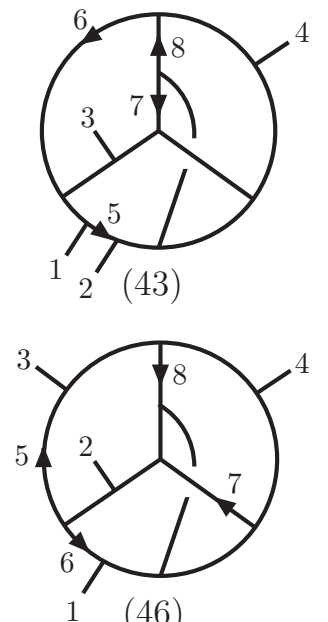

(46)

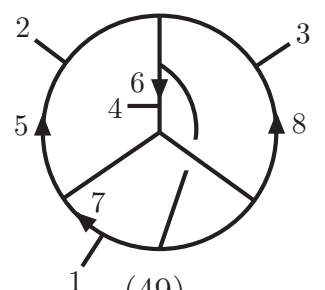

(49)

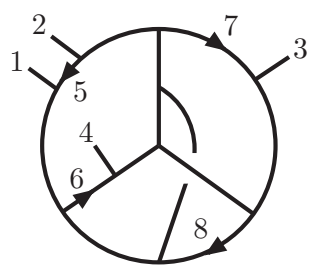

(41)
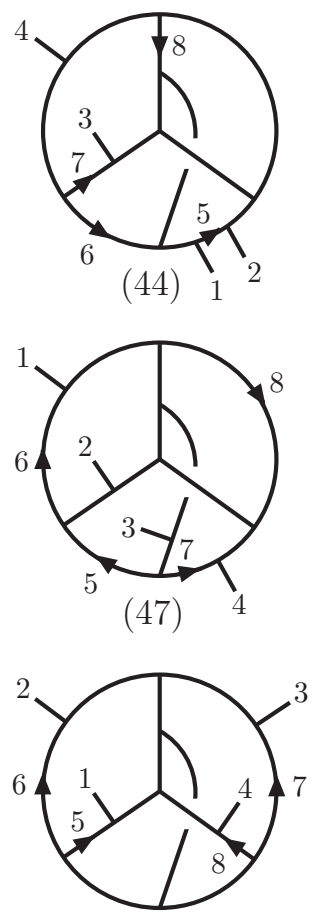

(50)

FIG. 8: Cubic graphs 39 to 50 that contribute to the four-loop four-point amplitude of $\mathcal{N}=4 \mathrm{sYM}$ theory and $\mathcal{N}=8$ supergravity. The labeling is the same as for fig. 5 .

and

$$
\left\{k_{2} \leftrightarrow k_{4}, l_{5} \leftrightarrow l_{6}, l_{7} \rightarrow k_{1}-l_{7}, l_{8} \rightarrow k_{3}-l_{8}\right\} .
$$

Imposing the invariance of numerator $N_{28}$ under eqs. (3.6) and (3.7) reduces the number of its unknown coefficients from 43 to 14 . The other master graph, graph 18, does not have any such automorphism relations; we are therefore left to determine a total of 57 parameters.

We then impose similar symmetry conditions on $N_{12}$, which may be written in terms of $N_{18}$ and $N_{28}$ as

$$
\begin{aligned}
N_{12}= & -N_{18}\left(k_{4}, k_{3}, k_{1}, l_{6},-l_{5},-l_{6}, l_{8}\right)+N_{18}\left(k_{4}, k_{3}, k_{2}, l_{6}, k_{2}+l_{8}, l_{5}, l_{7}\right) \\
& +N_{18}\left(k_{4}, k_{3}, k_{2}, k_{3}+l_{8}, l_{5}-l_{8},-l_{6}, l_{8}\right)-N_{28}\left(k_{1}, k_{2}, k_{3}, l_{5}-l_{8}, k_{3}-l_{6}+l_{8},-l_{6}, l_{8}\right) \\
& +N_{28}\left(k_{2}, k_{1}, k_{3},-l_{5}, 0,-l_{6}, l_{8}\right)-N_{28}\left(k_{4}, k_{3}, k_{1}, l_{6}-l_{8}, k_{2}-l_{5}+l_{8}, l_{8}, l_{8}\right) \\
& +N_{28}\left(k_{4}, k_{3}, k_{2}, k_{3}, k_{1}+l_{5},-k_{3}+l_{6}, l_{8}\right),
\end{aligned}
$$

by combining the $2 \mathrm{nd}, 6 \mathrm{th}, 14$ th and 21 st relations in eq. (A4) in appendix A. Invariance under the automorphisms of graph 12 fixes 37 parameters, leaving undetermined 20 param- 


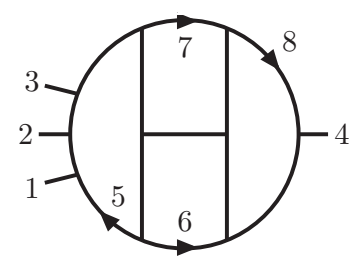

(51)

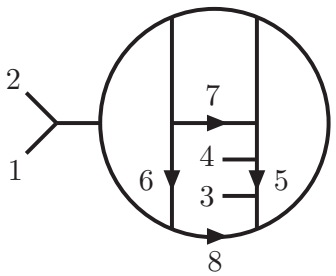

(54)

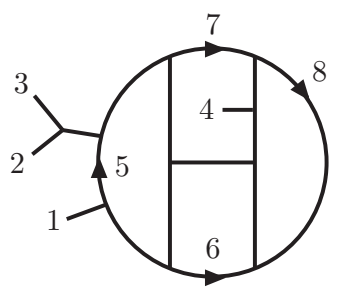

(57)

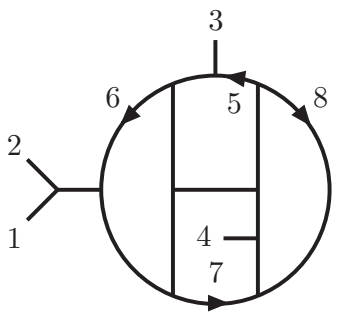

(60)

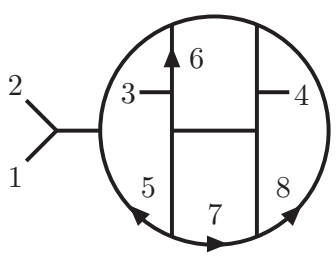

(63)

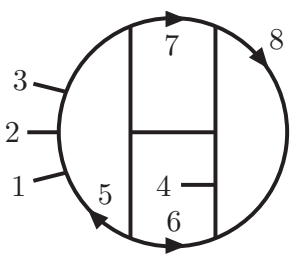

(52)

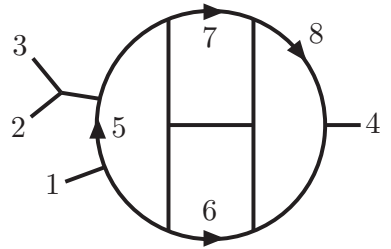

(55)

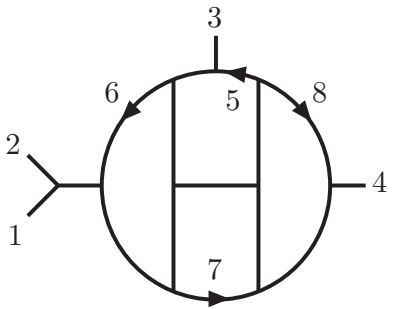

(58)

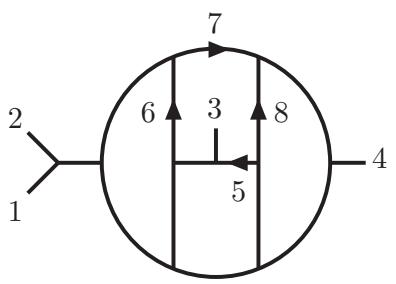

(61)

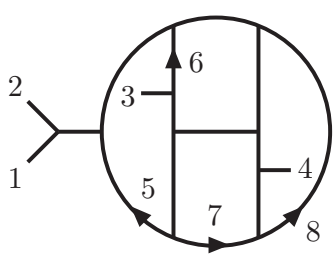

(64)

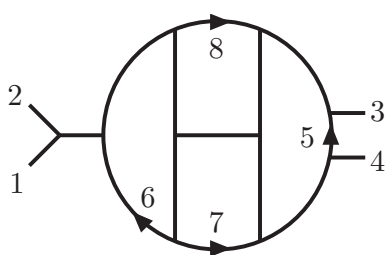

(53)

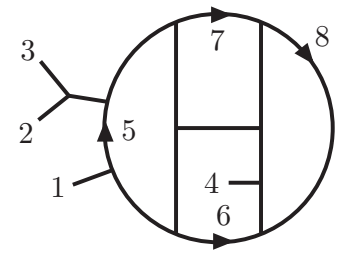

(56)

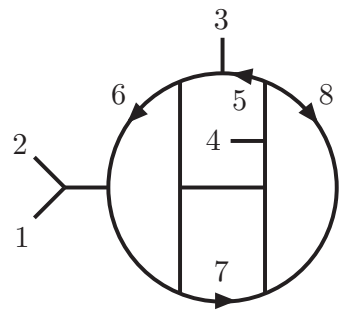

(59)

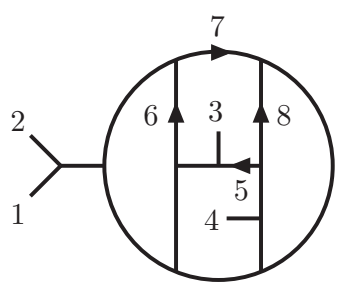

(62)

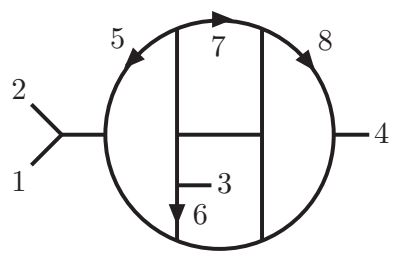

(65)

FIG. 9: Cubic graphs 51 to 65 that contribute to the four-loop four-point amplitude of $\mathcal{N}=4 \mathrm{sYM}$ theory and $\mathcal{N}=8$ supergravity. The labeling is the same as for fig. 5 .

eters. Similarly, imposing the graph symmetry condition on $N_{14}$ reduces the total number of unknown parameters to 17. (These parameter counts are for the specific set of duality relations given in eq. (A4). Using another set of relations would result in somewhat different parameter counts; however, the final solution would be the same.)

We could continue imposing more symmetry constraints on other numerators, but we already have a very small set of undetermined parameters. Ultimately, dynamical information provided by unitarity cuts should become necessary. Therefore we will now inspect 


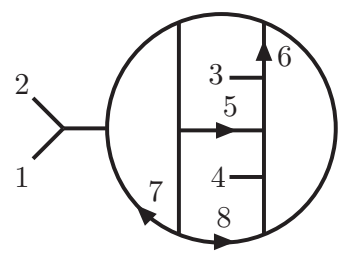

(66)

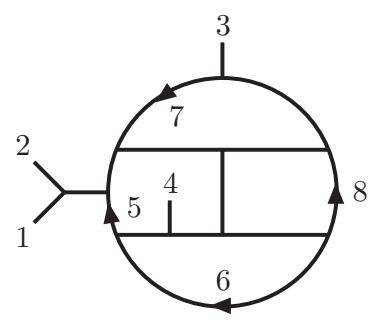

(69)

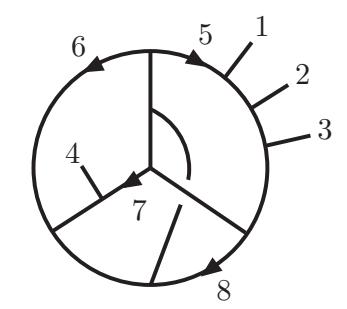

$(72)$

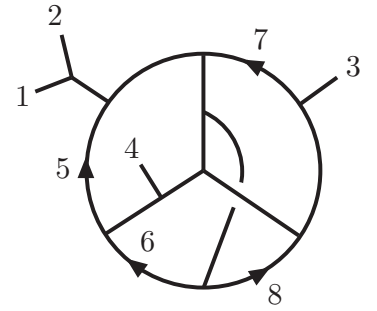

(75)

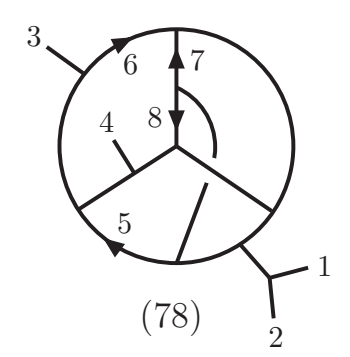

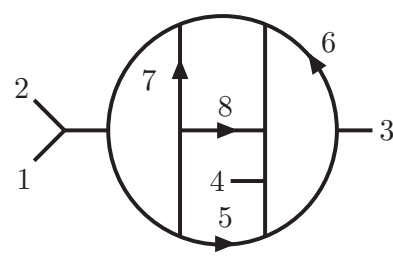

(67)

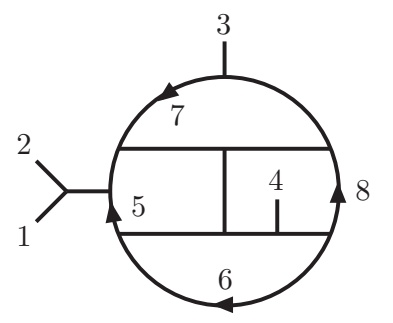

$(70)$

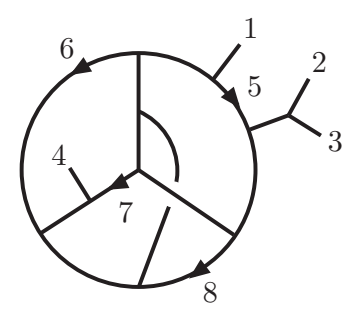

$(73)$

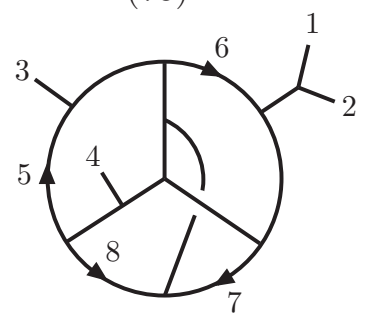

(76)

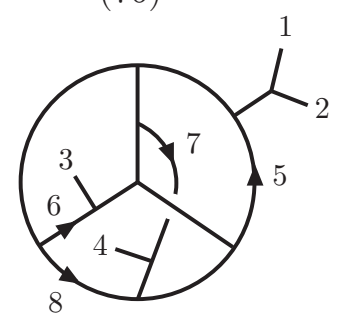

(79)

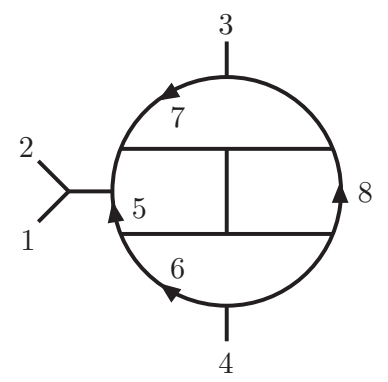

(68)

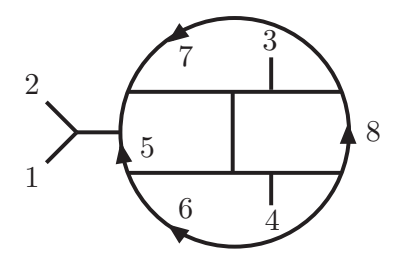

(71)

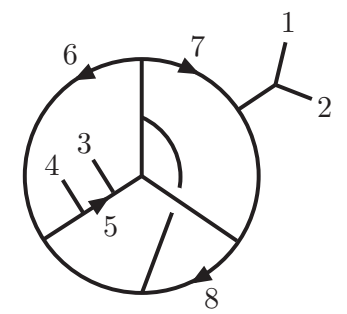

$(74)$

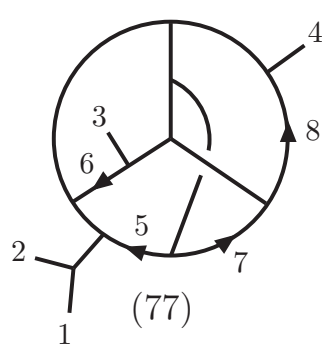

FIG. 10: Cubic graphs 66 to 79 that contribute to the four-loop four-point amplitude of $\mathcal{N}=4$ sYM theory and $\mathcal{N}=8$ supergravity. The labeling is the same as for fig. 5 .

some cuts. A good starting point is the maximal cut of graph 12. Its explicit value is easily obtained using the simple rung-rule numerator of that graph $[5,53]$,

$$
N_{12}^{\mathrm{rr}}=s^{2}\left(l_{5}+l_{6}+k_{1}+k_{4}\right)^{2} .
$$




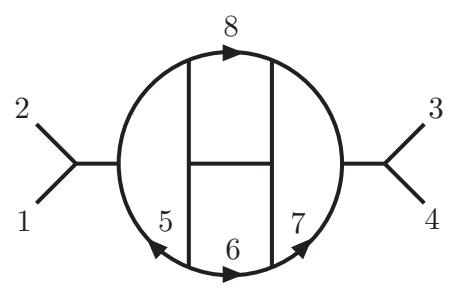

(80)

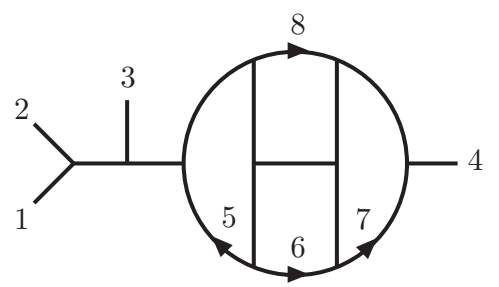

(83)

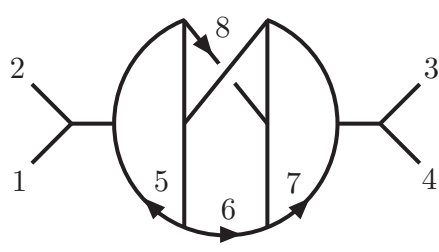

(81)

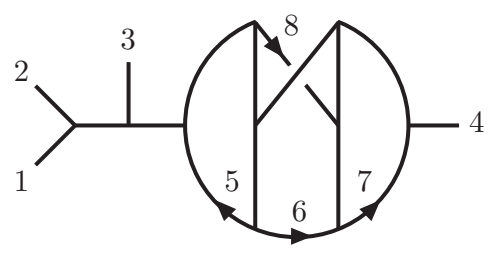

(84)

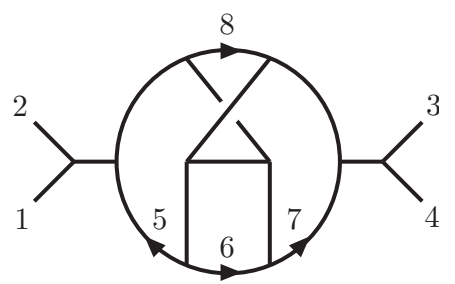

$(82)$

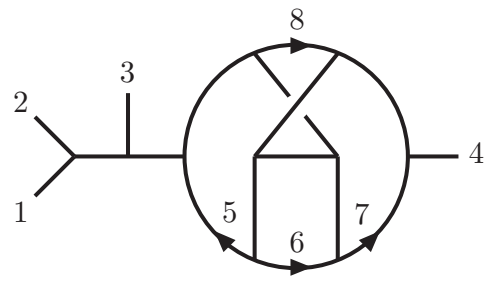

(85)

FIG. 11: Cubic graphs 80 to 85 that contribute to the four-loop four-point amplitude of $\mathcal{N}=4$ sYM theory. Graphs 83, 84, and 85 vanish identically for $\mathcal{N}=8$ supergravity, but carry an UV singularity in the $D=11 / 2 \mathcal{N}=4$ sYM case. The labeling is the same as for fig. 5 .

The rung rule was originally designed to reproduce iterated two-particle cuts. Since maximal cuts can be obtained from iterated two-particle cuts by imposing additional cut conditions, the rung rule reproduces the maximal cuts as well. Our task is to match $N_{12}$, as obtained from the duality relations, and $N_{12}^{\text {rr }}$ in eq. (3.9) on the maximal cut kinematics that uniquely single out this graph, i.e. we impose $p_{i}^{2}=0$ on all 13 propagators of graph 12 . Solving these conditions, we obtain

$$
\begin{aligned}
& l_{i}^{2}=0, \quad \tau_{15}=\tau_{25}=\tau_{36}=0, \quad \tau_{16}=-\tau_{26}, \quad \tau_{17}=-\tau_{57}, \\
& \tau_{38}=\tau_{68}, \quad \tau_{28}=\tau_{58}, \quad \tau_{27}=-\tau_{37}+\tau_{57}+\tau_{67}, \quad \tau_{18}=-s+\tau_{37}-\tau_{58}-\tau_{67}-\tau_{78} .
\end{aligned}
$$

Thus, on the maximal cut, $N_{12}^{\mathrm{rr}}$ becomes

$$
N_{12}^{\text {max. cut }}=s^{2}\left(t-\tau_{26}-\tau_{35}+\tau_{56}\right) .
$$

Requiring that the numerator obtained from the Ansatz (3.5) via eq. (3.8) matches $N_{12}^{\max \text {. cut }}$ on the maximal cut, the number of undetermined parameters is reduced from 17 to 8 .

Finally, all remaining parameters can be determined by requiring the next-to-maximal cut of graph 14, where all propagators except for $1 / l_{5}^{2}$ are placed on shell, to be satisfied. Graph 68 also contributes to this cut since it contains the same set of cut propagators. Relabeling graph 68 so it matches graph 14, and appropriately weighting the numerators by the remaining off-shell propagators, we find under the cut kinematics,

$$
N_{14}+\frac{l_{5}^{2}}{s}\left(N_{68} \mid \begin{array}{c}
\substack{l_{5} \rightarrow k_{1}-l_{5}, l_{6} \rightarrow-l_{6} \\
l_{7} \rightarrow-l_{7}, l_{8} \rightarrow-l_{8}} \\
\mid
\end{array}\right)=s\left(l_{5}+k_{2}+k_{3}\right)^{4} .
$$

The right-hand side of this equation is the numerator of graph 14, as constructed using the rung rule; in the rung-rule representation of the planar four-loop amplitude, graph 68 


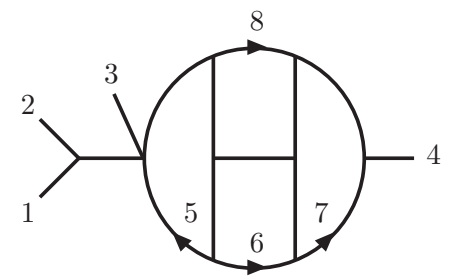

$\left(83^{\prime}\right)$

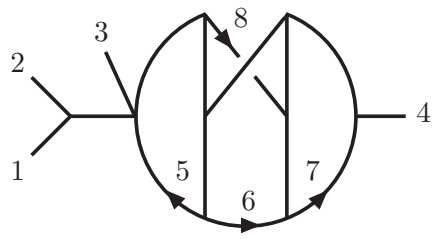

$\left(84^{\prime}\right)$

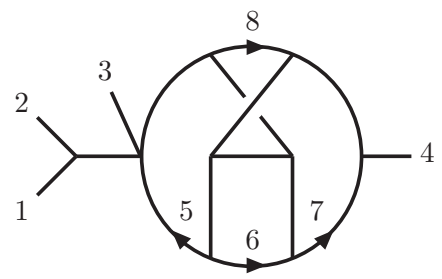

$\left(85^{\prime}\right)$

FIG. 12: The snail contributions in a form resolving the $0 / 0$ ambiguity of graphs 83,84 and 85 .

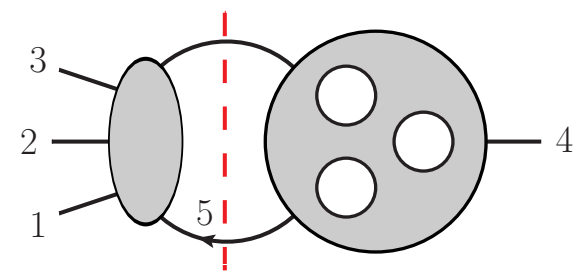

FIG. 13: A cut that determines the snail contribution in fig. $3(\mathrm{~d})$. In $\mathcal{N}=4 \mathrm{sYM}$ theory this cut vanishes at the level of the integrand.

vanishes [5]. This requirement fixes all remaining eight coefficients, giving us a unique expression for the master numerators,

$$
\begin{aligned}
N_{18}= & \frac{1}{4}\left(6 u^{2} \tau_{25}+u\left(2 s\left(5 \tau_{25}+2 \tau_{26}\right)-\tau_{15}\left(7 \tau_{16}+6 t\right)\right)\right. \\
& +t\left(\tau_{15} \tau_{26}-\tau_{25}\left(\tau_{16}+7 \tau_{26}\right)\right)+s\left(4 \tau_{15}\left(t-\tau_{26}\right)+6 \tau_{36}\left(\tau_{35}-\tau_{45}\right)\right. \\
& \left.\left.-\tau_{16}\left(4 t+5 \tau_{25}\right)-\tau_{46}\left(5 \tau_{35}+\tau_{45}\right)\right)+2 s^{2}\left(t+\tau_{26}-\tau_{35}+\tau_{36}+\tau_{56}\right)\right) \\
N_{28}= & \frac{1}{4}\left(s\left(2 \tau_{15} t+\tau_{16}\left(2 t-5 \tau_{25}+\tau_{35}\right)+5 \tau_{35}\left(\tau_{26}+\tau_{36}\right)+2 t\left(2 \tau_{46}-\tau_{56}\right)-10 u \tau_{25}\right)\right. \\
& \left.-4 s^{2} \tau_{25}-6 u\left(\tau_{46}\left(t-\tau_{25}+\tau_{45}\right)+\tau_{25} \tau_{26}\right)-t\left(\tau_{15}\left(4 \tau_{36}+5 \tau_{46}\right)+5 \tau_{25} \tau_{36}\right)\right) .
\end{aligned}
$$

It is quite striking that the solution we obtain is unique and relatively simple. There are presumably other solutions to the duality relations for this amplitude, but finding them would require relaxing some of the auxiliary constraints described in section II B.

This construction completely fixes the values of all numerators from $N_{1}$ to $N_{82}$ (subject to on-shell external kinematics) using the duality relations in appendix A.

\section{Resolving the snails}

In the previous subsection we obtained the numerators for the master graphs, which determine all numerator factors through duality relations. However, it does not resolve the 0/0 ambiguity appearing in graphs 83-85 in fig. 11, which needs at least one external momentum off shell to be properly defined, if we insist on representing the result in terms of graphs with only cubic vertices. As explained in section II D, these contributions are indeed finite, as can easily be confirmed explicitly using generalized unitarity cuts, as we do below.

First we consider the planar snail contributions to the four-loop four-point amplitude. To determine them, we evaluate the unitarity cut shown in fig. 13. In the planar limit, the integrals contributing to this cut are shown in fig. 14. Because the cut contains an on-shell 


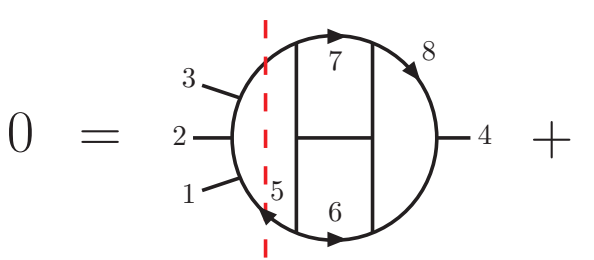

(51)

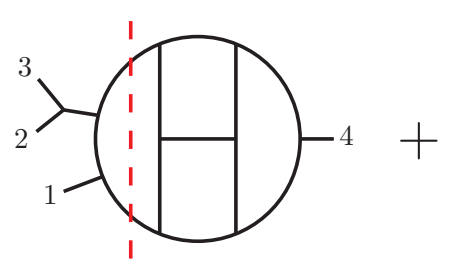

$(55)$

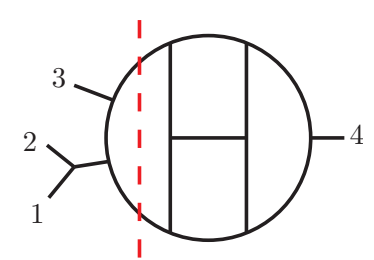

$(55)$

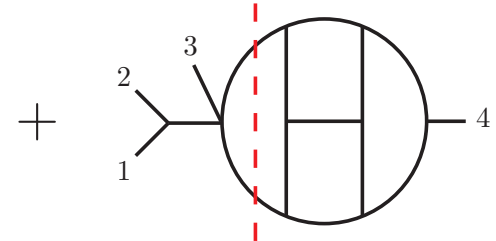

$\left(83^{\prime}\right)$

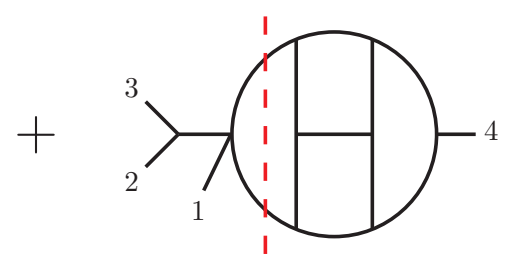

$\left(83^{\prime}\right)$

FIG. 14: The graphs contributing to the cut in fig. 13. The sum of these contributions to the cut vanishes in $\mathcal{N}=4 \mathrm{sYM}$ theory.

three-loop three-point subamplitude it must vanish, following the arguments in section IID. Therefore we can determine the contribution of the snail graph 83 in terms of that of graphs 51 and 55. Dressing the numerators of the first three graphs in fig. 14 with the appropriate ratios of propagators, we find that

$$
\begin{aligned}
N_{51}+ & \frac{\left(l_{5}-k_{1}-k_{2}\right)^{2}}{t}\left(\left.N_{55}\right|_{l_{5} \rightarrow l_{5}-k_{1}}\right)+\frac{\left(l_{5}-k_{1}\right)^{2}}{s}\left(\left.N_{55}\right|_{k_{1} \leftrightarrow k_{3}, l_{5} \rightarrow k_{1}+k_{2}-l_{5}}\right) \\
& =\frac{27}{2}\left(l_{5}-k_{1}\right)^{2}\left(l_{5}-k_{1}-k_{2}\right)^{2} u
\end{aligned}
$$

where we used the relations $l_{5}^{2} \rightarrow 0$ and $\tau_{45} \rightarrow 0$, valid on the cut. Here we relabeled the momenta of the two contributions of graph 55 (compare to fig. 9) to match the labels of graph 51. The two factors on the right-hand side of eq. (3.14) that depend on $l_{5}$ are simply inverse propagators belonging to graph 51. In order to compare to graph 83 we should remove these factors, because it does not have these propagators. Thus, in order for the sum of contributions in fig. 14 to vanish, we must have

$$
\frac{1}{s} N_{83^{\prime}}+\frac{1}{t}\left(\left.N_{83^{\prime}}\right|_{k_{1} \leftrightarrow k_{3}}\right)=-\frac{27}{2} u
$$

where we start with the labeling in fig. 12 and relabel accordingly. (Because the snail numerators turn out to have no loop-momentum dependence, we need only specify the external momentum relabelings.) Now numerator $N_{83^{\prime}}$ should respect the $1 \leftrightarrow 3$ antisymmetry of the graph. This constraint, together with eq. (3.15), implies that

$$
N_{83^{\prime}}=-\frac{9}{2} s(u-t)
$$

The original cubic graph in fig. 11 then has the regulated numerator factor,

$$
N_{83}=-\frac{9}{2} k_{4}^{2} s(u-t)
$$


for the snail graph 83 . Here $k_{4}^{2}$ should not be set to zero until after this factor has canceled the $1 / k_{4}^{2}$ propagator of the graph. The remaining two graphs, 84 and 85 , can be determined in an analogous fashion using a nonplanar cut which also isolates a three-point subamplitude. Alternatively, the duality relations in appendix A fix them to be $N_{85}=N_{84}=N_{83}$.

We have carried out a very similar analysis of the corresponding cuts of $\mathcal{N}=8$ supergravity and find that there are no snail contributions. This is in line with our heuristic expectations, described in section IID, that the gravity case should have an extra vanishing factor of $k_{i}^{2}$ in the numerator, setting all snail contributions to zero.

Now that we have a complete Ansatz for the amplitude, the remaining task is to confirm that it has all desired properties and that it is a correct representation of the amplitude. Indeed, we verified that all numerators respect the graph symmetries and that all duality constraints hold on the sYM numerators.

To prove that our construction is correct, we verified that a spanning set of $D$-dimensional generalized unitarity cuts are properly reproduced. It has been shown [11] that there are no contributions to the four-loop four-point scattering amplitude in $\mathcal{N}=4 \mathrm{sYM}$ theory beyond those exposed on next-to-next-to-maximal cuts. This result was later confirmed using sixdimensional cuts [34]. As long as a candidate representation manifestly contains no worse loop-momentum behavior for individual terms than the previous representation, as is true of our current form, the set of next-to-next-to-maximal cuts are spanning (complete). We employ those cuts to verify our new expression, by comparing it against the previous one.

We do not give the details here as the procedure is the same as given in refs. [11, 41], except that we generate the reference $D$-dimensional analytic cuts using the previouslyobtained forms of the amplitude [11]. For the gravity amplitude, obtained from the doublecopy formula (2.5), we confirmed that its cuts through (next-to) ${ }^{4}$-maximal cuts match the corresponding cuts of the result in ref. [10]. ${ }^{3}$ The agreement with the cuts of the earlier representation directly proves the duality and double-copy properties for the four-loop fourpoint amplitudes of $\mathcal{N}=4$ sYM theory and $\mathcal{N}=8$ supergravity.

\section{UV BEHAVIOR OF $\mathcal{N}=8$ SUPERGRAVITY AND $\mathcal{N}=4$ SYM THEORY}

In this section, we examine the UV properties of the four-loop four-particle $\mathcal{N}=4 \mathrm{sYM}$ theory and $\mathcal{N}=8$ supergravity amplitudes derived in the previous section, after reviewing lower-loop examples. Unlike the original form obtained for the $\mathcal{N}=8$ supergravity four-loop amplitude [10], the representation derived in this paper through eq. (2.5) is manifestly finite for $D<11 / 2$. This property makes it much easier to determine its UV behavior. We will see that, in complete harmony with the corresponding $\mathcal{N}=4$ sYM amplitude, a divergence is indeed present in the expected critical dimension $D_{c}=11 / 2$. In $\mathcal{N}=4 \mathrm{sYM}$ theory this divergence in the amplitude corresponds to a counterterm of the schematic form $\operatorname{Tr}\left(\mathcal{D}^{2} F^{4}\right)$, where $\mathcal{D}$ represents a covariant derivative and $F$ is the gauge field strength. Similarly, in $\mathcal{N}=8$ supergravity the amplitude divergence corresponds to a counterterm of the form $\mathcal{D}^{8} R^{4}$, with the indices of the four Riemann tensors arranged in the supersymmetric combination corresponding to the square of the Bel-Robinson tensor [65]. (For recent discussions of $\mathcal{D}^{2 k} R^{4}$ invariants in $\mathcal{N}=8$ supergravity see ref. [66].) We shall find a close connection

\footnotetext{
3 The need for checking this high an order arises because of the large numbers of loop momenta occurring
} in individual terms in the original representation of the $\mathcal{N}=8$ supergravity amplitude [10]. 
between coefficients of the UV counterterms in these theories.

In most of this section we will work in space-time dimension $D=D_{c}-2 \epsilon=11 / 2-2 \epsilon$. In the last subsection we will discuss the UV behavior of the color double-trace terms in the $\mathcal{N}=4$ sYM amplitude at four loops, for which the divergence in $D=11 / 2-2 \epsilon$ is not present. Here we move to the next dimension in which there can be a potential divergence, $D=6-2 \epsilon$, in order to assess whether there is one or not.

In $D=11 / 2-2 \epsilon$, there are no UV divergences for either $\mathcal{N}=4 \mathrm{sYM}$ or $\mathcal{N}=8$ supergravity below four loops; in both theories, the first divergences for $L=1,2,3$ are for dimensions $D_{c}=8,7,6$, respectively. Therefore there can be no UV subdivergences in $D=11 / 2-2 \epsilon$ at four loops. Near this dimension, the overall logarithmic UV divergences arise from integration regions in which the loop momenta are much larger than the external momenta. For the representations of the $\mathcal{N}=4$ sYM and $\mathcal{N}=8$ supergravity amplitudes in this paper, each integral is either finite or develops at most a logarithmic divergence. We can capture the leading UV behavior by expanding in small external momenta [67, 68]. The logarithmic behavior of each integral means that we need only the leading term in this expansion. The amplitudes in question reduce to a collection of vacuum integrals that are relatively easy to evaluate.

We begin by reviewing the UV properties of $\mathcal{N}=4$ sYM theory, in the light of the new representation of the four-loop four-point amplitude. Then we will turn to $\mathcal{N}=8$ supergravity.

\section{A. Review of UV behavior of $\mathcal{N}=4$ sYM theory}

The UV properties of $\mathcal{N}=4 \mathrm{sYM}$ theory at one through four loops were discussed in detail in ref. [11]. The first divergence appears in the critical dimension

$$
D_{c}=4+\frac{6}{L} \quad(L=2,3,4)
$$

and $D_{c}=8$ at $L=1$. In each case, the potential divergence is known to appear with a nonzero coefficient [11]. Thus the bound (4.1), proposed in refs. [8, 63], is in fact saturated, at least through four loops.

For gauge group $S U\left(N_{c}\right)$, gluon scattering amplitudes can be expressed in the trace basis, i.e. in terms of traces of products of generators in the fundamental representation. For four external gluons, only single-trace structures, of the form $\operatorname{Tr}\left(T^{a_{i}} T^{a_{j}} T^{a_{k}} T^{a_{l}}\right)$, and double-trace structures, of the form $\operatorname{Tr}\left(T^{a_{i}} T^{a_{j}}\right) \operatorname{Tr}\left(T^{a_{k}} T^{a_{l}}\right)$, can appear. At one and two loops, the color double-trace terms have the same UV behavior as the overall amplitudes, obeying eq. (4.1). However, starting at three loops they are less divergent [11]. (See also the discussions in refs. [64, 69].) In particular, the critical dimensions for finiteness for the double-trace terms satisfy

$$
D_{c}^{2 \text {-trace }}=4+\frac{8}{L} \quad(L=3,4) .
$$

Using the representations of the three- and four-loop amplitudes described in previous sections, we will see that these bounds follow more transparently than with the older representations in ref. [11]. That is because the color factors associated with the most divergent integrals now have a much simpler structure.

Ref. [11] showed that the bound (4.2) is saturated at three loops. This computation did not involve any UV subdivergences because $4+8 / 3=20 / 3<7$, and the first two-loop 


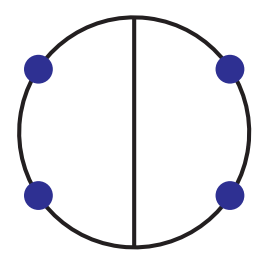

$V^{(\mathrm{P})}$

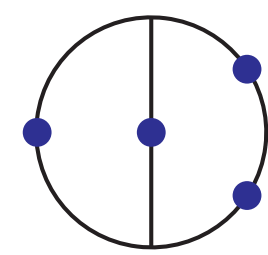

$V^{(\mathrm{NP})}$

FIG. 15: The two-loop vacuum integrals $V^{(\mathrm{P})}$ and $V^{(\mathrm{NP})}$. Each (blue) dot on a propagator indicates an additional power of the propagator.

divergences are at $D_{c}=7$. In section IVE we will show that the double-trace bound is saturated in the four-loop amplitude as well. In this case there are subdivergences, because $4+8 / 4=6$, and the first three-loop divergences are at $D_{c}=6$.

Before proceeding to three and four loops, we review the two-loop case. The two-loop four-gluon amplitude as given in ref. [8] already obeys the color-kinematic duality. The full amplitude was originally presented in the trace basis [53]; in the critical dimension $D_{c}=7$, its divergence in terms of vacuum integrals is [11]

$$
\begin{aligned}
\left.\mathcal{A}_{4}^{(2)}\right|_{\text {pole }} ^{S U\left(N_{c}\right)}=-g^{6} \mathcal{K} & {\left[\left(N_{c}^{2} V^{(\mathrm{P})}+12\left(V^{(\mathrm{P})}+V^{(\mathrm{NP})}\right)\right)\right.} \\
& \times\left(s\left(\operatorname{Tr}_{1324}+\operatorname{Tr}_{1423}\right)+t\left(\operatorname{Tr}_{1243}+\operatorname{Tr}_{1342}\right)+u\left(\operatorname{Tr}_{1234}+\operatorname{Tr}_{1432}\right)\right) \\
- & \left.12 N_{c}\left(V^{(\mathrm{P})}+V^{(\mathrm{NP})}\right)\left(s \operatorname{Tr}_{12} \operatorname{Tr}_{34}+t \operatorname{Tr}_{14} \operatorname{Tr}_{23}+u \operatorname{Tr}_{13} \operatorname{Tr}_{24}\right)\right]
\end{aligned}
$$

where $V^{(\mathrm{P})}$ and $V^{(\mathrm{NP})}$, shown in fig. 15, are the $k_{i}^{\mu} \rightarrow 0$ limit of the planar and nonplanar double-box integrals. ${ }^{4}$ Each blue dot denotes an extra power of the propagator on which it lies. Here each dot coincides with the location of an external leg attachment in the original four-point integral; adjacent propagators separated by an external momentum become equal as that momentum vanishes. The factor

$$
\mathcal{K} \equiv s t A^{\text {tree }}(1,2,3,4)
$$

contains the dependence on the external states. We use a shorthand notation for the color traces,

$$
\operatorname{Tr}_{i j k l} \equiv \operatorname{Tr}\left(T^{a_{i}} T^{a_{j}} T^{a_{k}} T^{a_{l}}\right), \quad \operatorname{Tr}_{i j} \equiv \operatorname{Tr}\left(T^{a_{i}} T^{a_{j}}\right)=\delta^{a_{i} a_{j}}
$$

The poles of the vacuum integrals $V^{(\mathrm{P})}$ and $V^{(\mathrm{NP})}$ in $D=7-2 \epsilon$ are $[8,11]$,

$$
\begin{aligned}
V^{(\mathrm{P})} & =-\frac{\pi}{20(4 \pi)^{7} \epsilon}, \\
V^{(\mathrm{NP})} & =-\frac{\pi}{30(4 \pi)^{7} \epsilon} .
\end{aligned}
$$

\footnotetext{
${ }^{4}$ We normalize our integrals as in ref. [11], so that at two loops there is a relative minus sign compared to the normalization in ref. [8].
} 
The UV divergence in the critical dimension, $D_{c}=7$, of the corresponding supergravity amplitude is [8]

$$
\left.\mathcal{M}_{4}^{(2)}\right|_{\text {pole }}=-2\left(\frac{\kappa}{2}\right)^{6} s t u\left(s^{2}+t^{2}+u^{2}\right) M_{4}^{\text {tree }}\left(V^{(\mathrm{P})}+V^{(\mathrm{NP})}\right) .
$$

We note that the UV divergence of the supergravity amplitude and that of the $1 / N_{c}^{2}$ suppressed single-trace sYM amplitude are given by the same linear combination of vacuum integrals, namely $V^{(\mathrm{P})}+V^{(\mathrm{NP})}$. We shall see that this pattern repeats itself through four loops. (This observation holds for the one-loop four-point amplitude as well. However, there the relation is rather trivial because both amplitudes are expressed in terms of the same scalar box integral; thus their UV divergences must be expressed in terms of a unique vacuum integral.)

We now turn to three loops. The integrals that appear in the three-loop four-point $\mathcal{N}=4$ sYM amplitude in the duality-satisfying form [30] all have 10 propagators. However, three of them, $I^{(\mathrm{j})}, I^{(\mathrm{k})}$ and $I^{(\mathrm{l})}$ in fig. 2, have one propagator depending solely on external momenta; we will refer to these integrals as nine-propagator integrals. From eqs. (2.15) and (2.7), their numerator factors are independent of the loop momenta: $N^{(\mathrm{j})}=N^{(\mathrm{k})}=N^{(\mathrm{l})}=(t-u) s / 3$.

If the other integrals had two powers of the loop momentum in the numerator, then they would have the same generic large loop-momentum behavior as the nine-propagator integrals. However, the other integrals all have numerators that are at most linear in the loop momenta. Equation (2.19) shows that the numerator $N^{(\mathrm{e})}$ for the master graph (e) is linear in the $\tau_{i j}$, which are in turn linear in the loop momenta. The Jacobi relations (2.7) preserve this linearity for all other numerators. (In some cases the linear dependence cancels down to a constant behavior.) Therefore the leading UV divergence of the three-loop $\mathcal{N}=4$ sYM amplitude comes from the three nine-propagator integrals.

This result is consistent with a rearrangement of the leading UV terms of the earlier representation, as discussed in ref. [11]. Because graphs $(\mathrm{j}),(\mathrm{k})$ and $(\mathrm{l})$ contain an external three-point tree, their color factors must be proportional to the product of two structure constants, multiplied by a color Casimir operator which has no free color indices [11]. The product of the two structure constants, $\tilde{f}^{a_{1} a_{2} b} \tilde{f}^{b a_{3} a_{4}}$ or a permutation thereof, takes the same form as a tree amplitude, namely a single color trace. Hence the leading UV divergence at three loops, in the critical dimension $D_{c}=4+6 / 3=6$, contains no double color-trace terms [11], while they are present in the leading divergence at two loops, eq. (4.6). The duality-satisfying representation of the amplitude automatically has the no-leading-doubletrace feature. Very similar behavior is observed within a string theory analysis [64]. There the leading behavior at three (and four) loops is dominated by the collision of pairs of vertex operators, producing inverse momentum factors, reminiscent of the form of graphs $(\mathrm{j}),(\mathrm{k})$ and (1). The finiteness of the three-loop double-trace terms in $D=6$ remains puzzling from the point of view of field-theoretic algebraic nonrenormalization considerations [69].

To extract the UV divergence, we carry out the small momentum expansion [67, 68]. Integral $(\mathrm{j})$ reduces to the vacuum integral $V^{(\mathrm{A})}[11,55]$ displayed in fig. 16, while integrals (k) and (l) reduce to $V^{(\mathrm{B})}$. These integrals diverge first in $D=6$. Their color factors are closely related: We can use a color Jacobi identity involving the boxes in the upper right-hand corner of graphs $(\mathrm{j})$ and $(\mathrm{k})$ of fig. 2 to show that the difference between $C_{(\mathrm{j})}$ and $C_{(\mathrm{k})}$ contains a triangle subgraph. A further color Jacobi identity allows us to replace the triangle by a three-vertex, multiplied by the quadratic Casimir factor $C_{A} / 2=N_{c}$. Iterating 


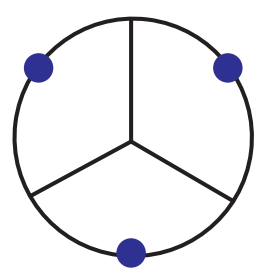

$V^{(\mathrm{A})}$

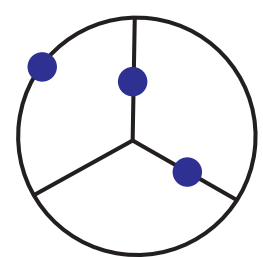

$V^{(\mathrm{B})}$

FIG. 16: The three-loop vacuum integrals $V^{(\mathrm{A})}$ and $V^{(\mathrm{B})}$.

this procedure, and also applying it to the difference of $C_{(\mathrm{j})}$ and $C_{(\mathrm{l})}$, we find that

$$
C_{(\mathrm{j})}-N_{c}^{3} \tilde{f}^{a_{1} a_{2} b} \tilde{f}^{b a_{3} a_{4}}=C_{(\mathrm{k})}=C_{(\mathrm{l})} .
$$

Note that $C_{(\mathrm{k})}$ and $C_{(1)}$ are associated with nonplanar graphs, and hence have only subleading-color terms. Equation (4.8) states that the subleading-color parts of $C_{(\mathrm{j})}, C_{(\mathrm{k})}$ and $C_{(1)}$ are all equal.

Taking into account that the combinatorial factor of $I^{(\mathrm{l})}$ is twice as large as those for $I^{(\mathrm{j})}$ and $I^{(\mathrm{k})}$ in fig. 2, and expressing the color factors for $(\mathrm{j}),(\mathrm{k})$ and $(\mathrm{l})$ in the trace basis, it is straightforward to see that the UV divergence in $D_{c}=6$ is [11],

$$
\begin{aligned}
\left.\mathcal{A}_{4}^{(3)}(1,2,3,4)\right|_{\text {pole }} ^{S U\left(N_{c}\right)} & =2 g^{8} \mathcal{K}\left(N_{c}^{3} V^{(\mathrm{A})}+12 N_{c}\left(V^{(\mathrm{A})}+3 V^{(\mathrm{B})}\right)\right) \\
\times & \left(s\left(\operatorname{Tr}_{1324}+\operatorname{Tr}_{1423}\right)+t\left(\operatorname{Tr}_{1243}+\operatorname{Tr}_{1342}\right)+u\left(\operatorname{Tr}_{1234}+\operatorname{Tr}_{1432}\right)\right) .
\end{aligned}
$$

The UV poles of the vacuum graphs are [55]

$$
\begin{aligned}
\left.V^{(\mathrm{A})}\right|_{\text {pole }} & =-\frac{1}{6(4 \pi)^{9} \epsilon}, \\
\left.V^{(\mathrm{B})}\right|_{\text {pole }} & =-\frac{1}{6(4 \pi)^{9} \epsilon}\left(\zeta_{3}-\frac{1}{3}\right) .
\end{aligned}
$$

We may compare this result to the UV divergence in $D_{c}=6$ of the corresponding $\mathcal{N}=8$ supergravity amplitude [55],

$$
\left.\mathcal{M}_{4}^{(3)}\right|_{\text {pole }}=-\left(\frac{\kappa}{2}\right)^{8}(s t u)^{2} M_{4}^{\text {tree }}\left[10\left(V^{(\mathrm{A})}+3 V^{(\mathrm{B})}\right)\right] .
$$

In this case the leading UV divergence involves not only the $1 \mathrm{PR}$ nine-propagator integrals, but also the 1PI ten-propagator integrals whose sYM numerators are linear in the loop momenta, because their (squared) supergravity numerators are quadratic. Once again, as was true for the two-loop amplitude, the UV divergence of the three-loop supergravity amplitude and that of the $1 / N_{c}^{2}$-suppressed single-color-trace three-loop sYM amplitude are given by the same combination of vacuum integrals, namely $V^{(\mathrm{A})}+3 V^{(\mathrm{B})}$. Below we shall see that the same phenomenon persists through four loops.

Using the representation of the four-loop four-point $\mathcal{N}=4$ sYM amplitude derived in earlier sections it is equally straightforward to recover its UV divergence. Indeed, an inspection of the integrals listed in figs. 5-12 and of their numerator factors listed in appendix B 

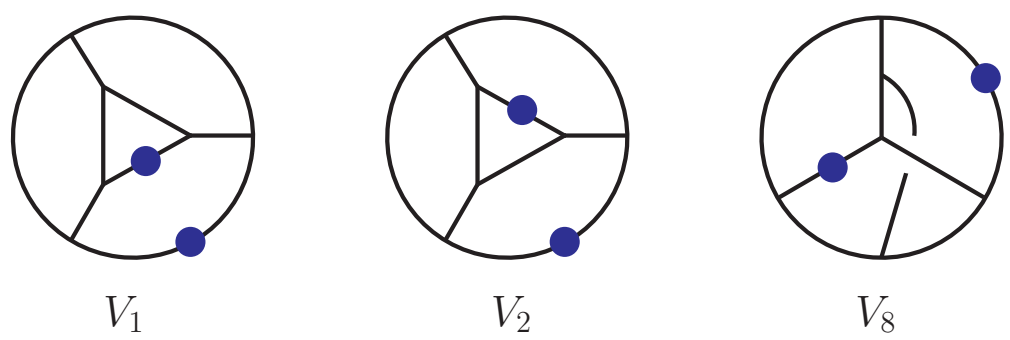

FIG. 17: The basic four-loop vacuum integrals $V_{1}, V_{2}$ and $V_{8}$, to which all others can be reduced.

reveals that the leading UV behavior comes solely from integrals $I_{80}$ through $I_{85}$. These six integrals have 11 internal propagators, and numerator factors that are independent of the loop momentum. Therefore they diverge first in $D_{c}=11 / 2$, which matches the expected critical dimension, $D_{c}=4+6 / L$ with $L=4$.

In contrast, the $1 \mathrm{PI}$ integrals $I_{1}$ through $I_{52}$ have 13 internal propagators. Their numerators would have to be quartic in the loop momenta for them to diverge in $D=11 / 2$. However, we note from eq. (3.14) that the master numerators $N_{18}$ and $N_{28}$ are quadratic in the $\tau_{i j}$, and hence merely quadratic in the loop momenta. The Jacobi relations (A4) and (A5) preserve this quadratic behavior for all numerators. Therefore integrals $I_{1}$ through $I_{52}$ are finite in $D_{c}=11 / 2$. The $1 \mathrm{PR}$ integrals $I_{53}$ through $I_{79}$ have 12 internal propagators. If their numerators were quadratic in the loop momenta, then they would diverge in $D=11 / 2$. However, it is easy to see from eqs. (A5) and (B1) that their numerators are all linear in the loop momenta.

Integrals $I_{80}$ through $I_{85}$ reduce easily to vacuum integrals in the limit that the external momenta vanish. The planar integrals $I_{80}$ and $I_{83}$ reduce to the vacuum integral $V_{1}$ depicted in fig. 17. While integrals $I_{81}$ and $I_{84}$ are nonplanar as four-point graphs, in the vacuum limit they reduce to the planar vacuum integral $V_{2}$. Finally, integrals $I_{82}$ and $I_{85}$ reduce to the nonplanar vacuum integral $V_{8}$.

As was the case at three loops, the color factors for the leading UV graphs are related by color Jacobi identities. In this case we can subtract, for example, $C_{81}$ from $C_{80}$, and use a Jacobi identity operating on the box at the top center of the graphs in fig. 11 . Then we reduce the resulting triangle subgraphs iteratively, to find

$$
C_{80,83}-2 N_{c}^{4} \tilde{f}^{a_{1} a_{2} b} \tilde{f}^{b a_{3} a_{4}}=C_{81}=C_{82}=C_{84}=C_{85} .
$$

Again, the subleading-color parts of all contributing color factors are equal.

From eq. (A5), the numerator factors obey $N_{80}=N_{81}=N_{82}$ and $N_{83}=N_{84}=N_{85}$. On the other hand, the combinatorial factor of $I_{81}$ in eq. (3.3) is twice as large as those for $I_{80}$ and $I_{82}$, and similarly for $I_{84}$ with respect to $I_{83}$ and $I_{85}$. Taking into account both combinatorial and numerator factors, the contribution of $I_{83}$ is $-\frac{9}{8}$ times that of $I_{80}$, and similarly for the other two pairs of graphs. Combining all terms and switching to the colortrace basis, we find that the UV divergence in the critical dimension $D=11 / 2$ is given by,

$$
\begin{aligned}
\left.\mathcal{A}_{4}^{(4)}(1,2,3,4)\right|_{\text {pole }} ^{S U\left(N_{c}\right)} & =-6 g^{10} \mathcal{K} N_{c}^{2}\left(N_{c}^{2} V_{1}+12\left(V_{1}+2 V_{2}+V_{8}\right)\right) \\
& \times\left(s\left(\operatorname{Tr}_{1324}+\operatorname{Tr}_{1423}\right)+t\left(\operatorname{Tr}_{1243}+\operatorname{Tr}_{1342}\right)+u\left(\operatorname{Tr}_{1234}+\operatorname{Tr}_{1432}\right)\right)
\end{aligned}
$$


in agreement with the results of ref. [11]. It is interesting to note from eq. (4.14) that the single-trace UV divergence in $D=11 / 2$ has $N_{c}^{4}$ and $N_{c}^{2}$ components, but the $N_{c}^{0}$ component vanishes. There is currently no general explanation for this fact, apart from the explicit values of the color factors $C_{80}$ through $C_{85}$.

The values of the three master integrals appearing in eq. (4.14) are [11]

$$
\begin{aligned}
& V_{1}=\frac{1}{(4 \pi)^{11} \epsilon}\left[\frac{512}{5} \Gamma^{4}\left(\frac{3}{4}\right)-\frac{2048}{105} \Gamma^{3}\left(\frac{3}{4}\right) \Gamma\left(\frac{1}{2}\right) \Gamma\left(\frac{1}{4}\right)\right]+\mathcal{O}(1), \\
& V_{2}=\frac{1}{(4 \pi)^{11} \epsilon}\left[-\frac{4352}{105} \Gamma^{4}\left(\frac{3}{4}\right)+\frac{832}{105} \Gamma^{3}\left(\frac{3}{4}\right) \Gamma\left(\frac{1}{2}\right) \Gamma\left(\frac{1}{4}\right)\right]+\mathcal{O}(1), \\
& V_{8}=\frac{1}{(4 \pi)^{11} \epsilon}\left[-\frac{20992}{2625} \Gamma^{4}\left(\frac{3}{4}\right)+\frac{128}{75} \Gamma^{3}\left(\frac{3}{4}\right) \Gamma\left(\frac{1}{2}\right) \Gamma\left(\frac{1}{4}\right)+\frac{8}{21 \Gamma\left(\frac{3}{4}\right)} \mathrm{NO}_{m}\right]+\mathcal{O}(1),
\end{aligned}
$$

where $\mathrm{NO}_{m}$ denotes a certain three-loop two-point nonplanar integral. While its analytic expression in $D=11 / 2$ is not known, it may be evaluated numerically [11] using the Gegenbauer polynomial $x$-space technique (GPXT) [70], with the result $\mathrm{NO}_{m}=-6.1983992267 \ldots$ We remark that an evaluation to much higher accuracy is also possible [71] using the DRA method [72], which involves combining dimensional recurrence relations [73] with analyticity in the space-time dimension. (This latter method has been applied to similar, and even more complex, integrals in refs. [74].)

In the following two subsections we will obtain the UV divergence of the corresponding $\mathcal{N}=8$ supergravity amplitude. We will find that, as for the two- and three-loop case, it is given by the same combination of vacuum integrals as the $1 / N_{c}^{2}$-suppressed single-trace UV divergence of the corresponding four-point $\mathcal{N}=4 \mathrm{sYM}$ amplitude, namely $V_{1}+2 V_{2}+V_{8}$.

\section{B. $\mathcal{N}=8$ supergravity vacuum graphs at four loops}

With the duality-satisfying form of the corresponding $\mathcal{N}=4$ sYM amplitude as the starting point, the double-copy formula (2.5) immediately gives us an expression for the four-loop $\mathcal{N}=8$ supergravity integrand. We confirmed this integrand by comparing its cuts with the cuts of the known four-loop $\mathcal{N}=8$ amplitude [10]. The double-copy formula is equivalent to the following squaring relation for the $\mathcal{N}=8$ supergravity numerators:

$$
N_{i}^{\mathcal{N}=8}=\left(N_{i}^{\mathcal{N}=4}\right)^{2} .
$$

By inspecting the squares of the numerator factors listed in appendix B, and counting the number of loop momenta in the numerator of each integral, it is easy to see that, in all cases, the integrals composing the resulting $\mathcal{N}=8$ supergravity amplitude are manifestly finite for $D<11 / 2$.

Indeed, as remarked in the previous subsection, the maximum degree in loop momenta of the numerator factors of the sYM amplitude is 2 for the 13-propagator integrals, 1 for the 12-propagator integrals and 0 for the 11-propagator integrals, where we count only those propagators carrying loop momentum. Consequently, the maximum degree in the loop momenta of each supergravity numerator polynomial is 4, 2 and 0 for the 13-, 12- and 11-propagator integrals, respectively. Such integrals all generically diverge logarithmically in $D=11 / 2$. Thus, the worst UV behavior of any $\mathcal{N}=8$ supergravity integral matches that 
of the worst-behaved integrals for the $\mathcal{N}=4 \mathrm{sYM}$ amplitude, i.e. the 11-propagator graphs (80)-(85) of fig. 11, which have the form of propagator corrections. The main difference is that now graphs other than propagator corrections carry this leading behavior. The representation of the four-loop amplitude described here reproduces the finiteness bounds of ref. [10], but the UV behavior is now manifest, allowing us to avoid performing any loop integration to expose this feature.

The new representation also makes it far simpler to determine whether the finiteness bound $D<11 / 2$ is saturated, by extracting the precise UV divergence of $\mathcal{N}=8$ supergravity in $D_{c}=11 / 2$. In our earlier representation [10], the UV divergence required the sixth-order terms in the expansion in small external momenta, making it rather cumbersome to extract. Now that the UV behavior is manifest, only the leading term in the expansion is required. This feature means that for each integral, we need only retain the terms in each sYM numerator with the highest powers of the loop momenta, and then square them.

The result of the expansion in the external momenta is a collection of tensor integrals, in which the numerator factors have a homogeneous degree in the loop momenta and are polynomials in the scalar products of loop and external momenta. Such integrals may be further reduced by making use of Lorentz invariance in order to extract the dependence on the external momenta from the tensor integrals. More precisely, under integration we can replace a generic two-tensor by

$$
l_{i}^{\mu_{i}} l_{j}^{\mu_{j}} \mapsto \frac{1}{D} \eta^{\mu_{i} \mu_{j}} l_{i} \cdot l_{j}
$$

and a four-tensor by

$$
l_{i}^{\mu_{i}} l_{j}^{\mu_{j}} l_{k}^{\mu_{k}} l_{l}^{\mu_{l}} \mapsto \frac{1}{(D-1) D(D+2)}\left(A \eta^{\mu_{i} \mu_{j}} \eta^{\mu_{k} \mu_{l}}+B \eta^{\mu_{i} \mu_{k}} \eta^{\mu_{j} \mu_{l}}+C \eta^{\mu_{i} \mu_{l}} \eta^{\mu_{j} \mu_{k}}\right),
$$

where

$$
\begin{aligned}
& A=(D+1) l_{i} \cdot l_{j} l_{k} \cdot l_{l}-l_{i} \cdot l_{k} l_{j} \cdot l_{l}-l_{i} \cdot l_{l} l_{j} \cdot l_{k}, \\
& B=-l_{i} \cdot l_{j} l_{k} \cdot l_{l}+(D+1) l_{i} \cdot l_{k} l_{j} \cdot l_{l}-l_{i} \cdot l_{l} l_{j} \cdot l_{k}, \\
& C=-l_{i} \cdot l_{j} l_{k} \cdot l_{l}-l_{i} \cdot l_{k} l_{j} \cdot l_{l}+(D+1) l_{i} \cdot l_{l} l_{j} \cdot l_{k} .
\end{aligned}
$$

Upon using these identities and summing over all permutations we find that the UV pole has the form,

$$
\left.\mathcal{M}_{4}^{(4)}\right|_{\text {pole }}=-\frac{1}{4}\left(\frac{\kappa}{2}\right)^{10} s t u\left(s^{2}+t^{2}+u^{2}\right)^{2} M_{4}^{\text {tree }} \widetilde{\mathcal{V}}^{(4)}
$$

where $M_{4}^{\text {tree }}$ is the four-point tree-level supergravity amplitude, and

$$
\widetilde{\mathcal{V}}^{(4)}=\sum_{i=1}^{69} I_{i}^{v}
$$

is the sum of integrals $I_{i}^{v}$ shown in figs. 18-20. All the kinematic dependence has been extracted in eq. (4.20); each integral $I_{i}^{v}$ is a pure number multiplied by a $1 / \epsilon$ pole. The numerator factors for these integrals are given in the first column of table I (not counting the column labeling the integrals $\left.I_{i}^{v}\right)$ in appendix C. The overall kinematic factor of $\left(s^{2}+t^{2}+u^{2}\right)^{2}$ is guaranteed by complete permutation symmetry of the four-point amplitude. 


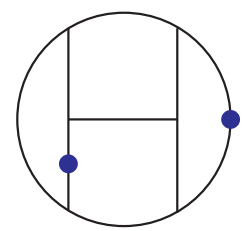

(1)

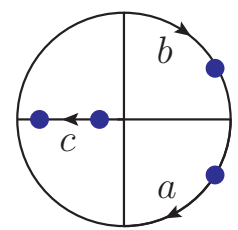

(6)

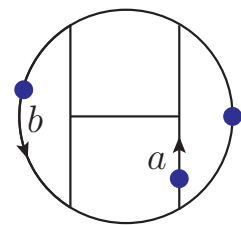

(11)

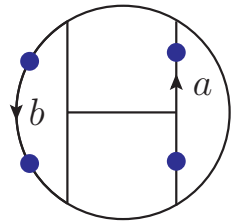

(16)

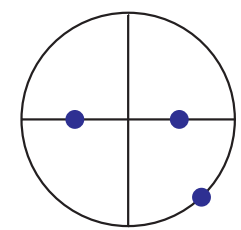

(21)

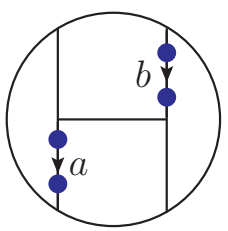

(2)

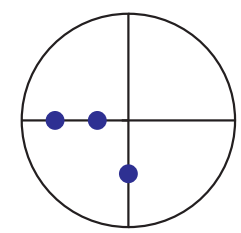

(7)

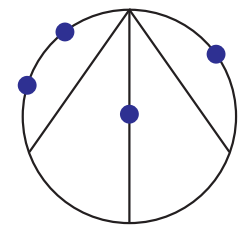

(12)

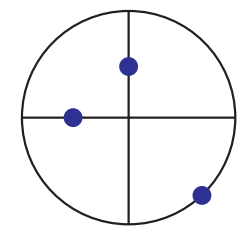

(17)

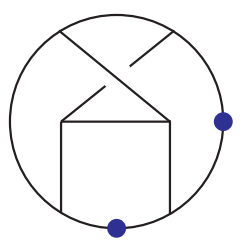

(22)

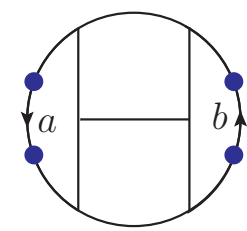

(3)

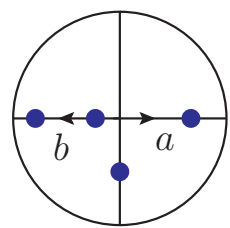

(8)

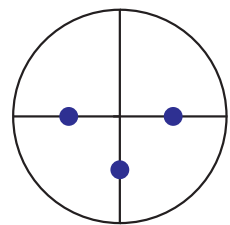

(13)

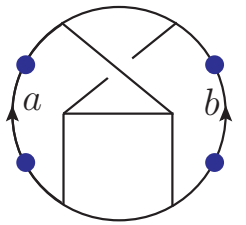

(18)

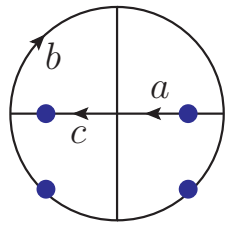

(23)

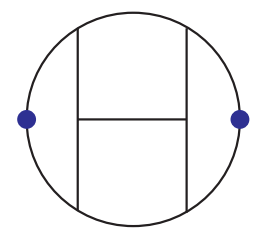

(4)

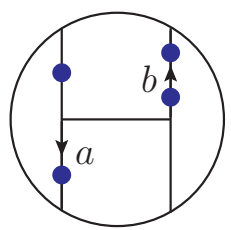

(9)

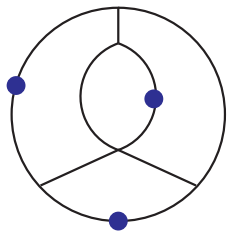

(14)

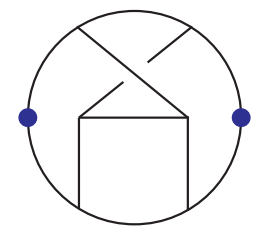

(19)

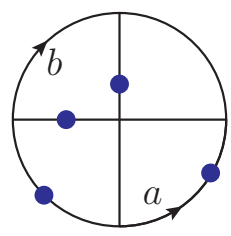

(24)

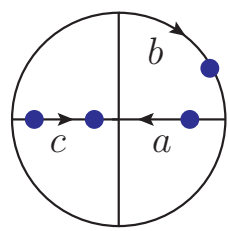

(5)

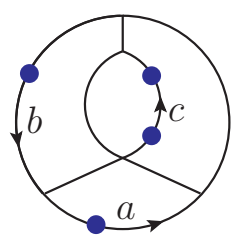

(10)

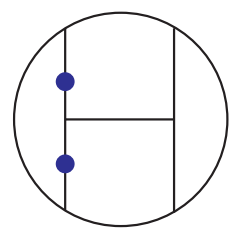

(15)

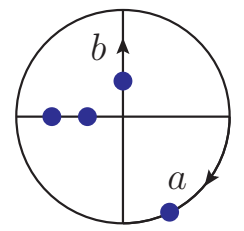

(20)

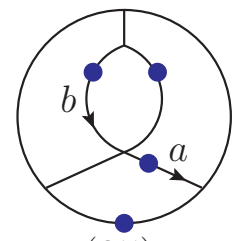

(25)

FIG. 18: Vacuum graphs $I_{1}^{v}$ through $I_{25}^{v}$. The momentum labels refer to the internal lines carrying an arrow. Their numerator factors are listed in table I in appendix C.

The precise combination of vacuum integrals $I_{i}^{v}$, in particular their numerical coefficients and numerator tensor structures, is sensitive to the choice of independent loop momenta in each of the integrals appearing in the complete supergravity amplitude. This choice is inherited in turn from the parametrization of the integrals of the $\mathcal{N}=4$ sYM amplitude, through the squaring relation (4.16). The ambiguity in choosing the loop momenta may also be used to generate identities between different integrals.

We now discuss in some more detail how we arrived at the results in the first column of table I in appendix C. After applying eqs. (4.17) and (4.18), the numerator factors of the vacuum integrals appearing in $\widetilde{\mathcal{V}}^{(4)}$, which no longer carry any external momenta, can be simplified further using loop-momentum conservation. Often, inverse propagators can be 


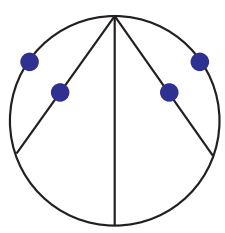

(26)

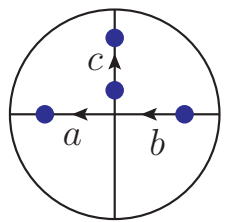

(31)

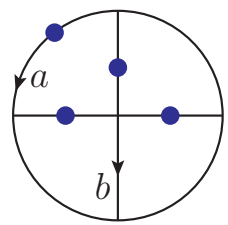

(36)

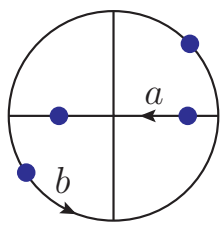

(41)

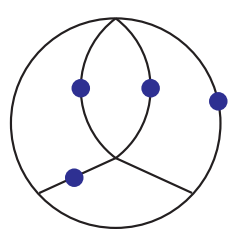

(46)

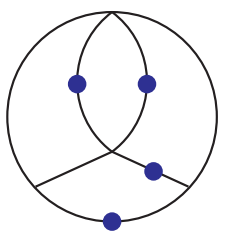

(27)

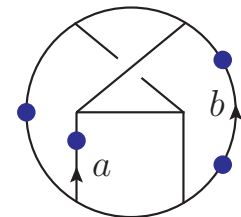

(32)

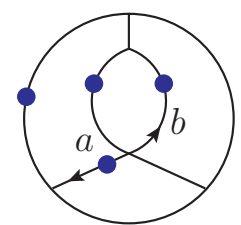

(37)

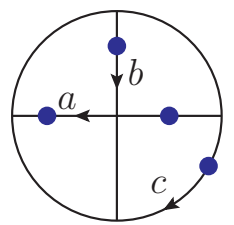

(42)

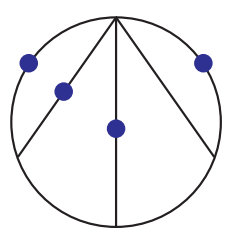

(47)

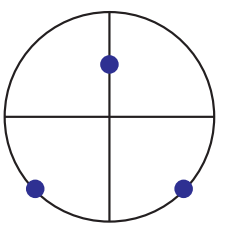

(28)

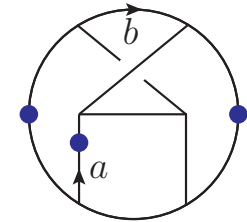

(33)

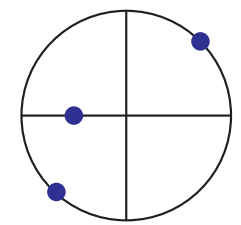

(38)

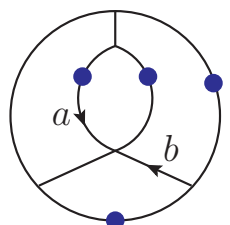

(43)

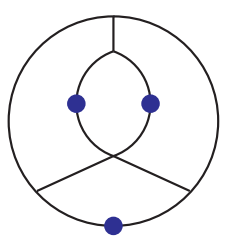

(48)

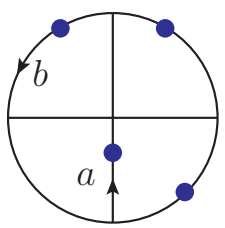

(29)

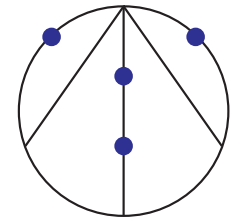

(34)

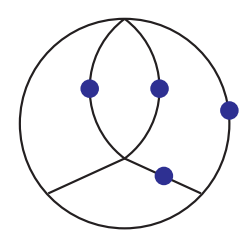

(39)

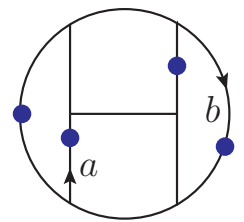

(44)

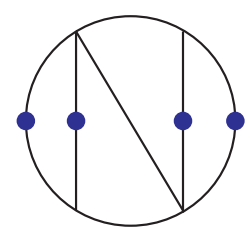

(49)

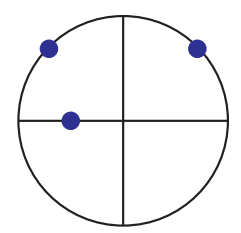

(30)

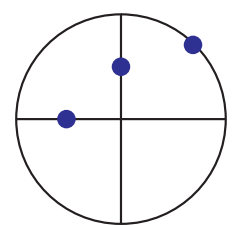

(35)

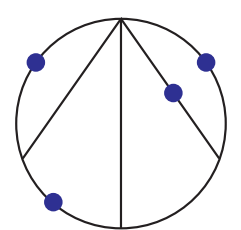

(40)

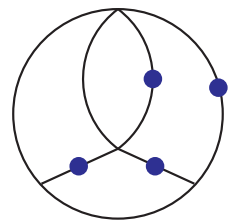

(45)

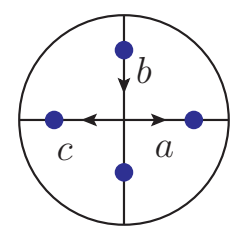

(50)

FIG. 19: Vacuum graphs $I_{26}^{v}$ through $I_{50}^{v}$.

identified, using for example $l_{i} \cdot l_{j}=\frac{1}{2}\left(l_{k}^{2}-l_{i}^{2}-l_{j}^{2}\right)$ when $l_{i}+l_{j}=l_{k}$ corresponds to a propagator for the graph. Such factors will cancel existing propagators and lead to simpler integrals with fewer propagators and lower-degree numerator factors. In carrying out this procedure, it is useful to ensure that propagators are not 'over-canceled'. For example, no squared inverse propagator should appear in a vacuum integral that does not have the corresponding propagator raised to at least the second power. For certain integrals the complete numerator factor may be written as a combination of different inverse propagators. In this case, the integral reduces to a combination of scalar integrals of different topologies, each of which is obtained from the initial integral by collapsing some of its propagators. For other integrals, the rank of the original numerator tensor is reduced by only two units. For 13 integrals the numerator factor remains of fourth order in loop momenta with no canceled propagators; in all such cases the numerator factors carrying loop momenta may be expressed as a perfect 


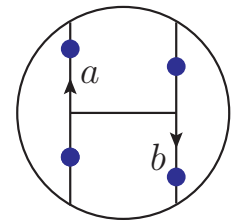

(51)

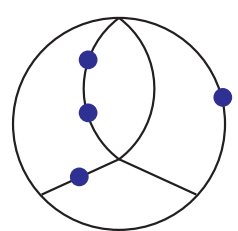

(56)

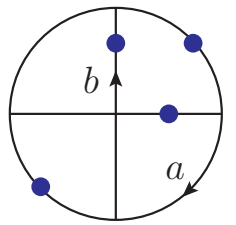

(61)

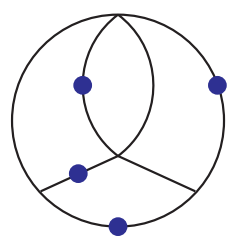

(66)

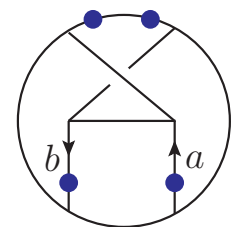

(52)

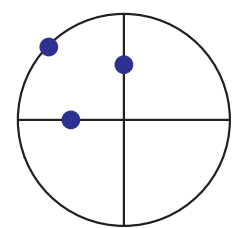

(57)

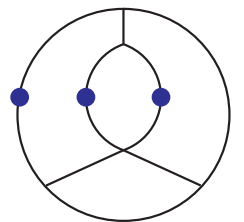

(62)

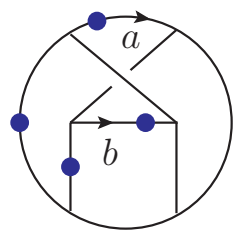

(67)

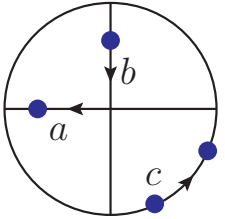

(53)

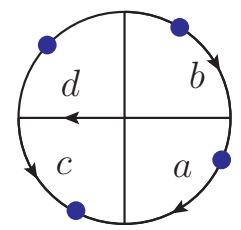

(58)

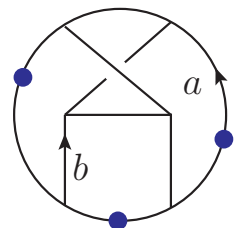

(63)

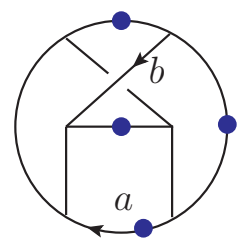

(68)

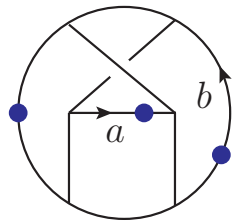

(54)

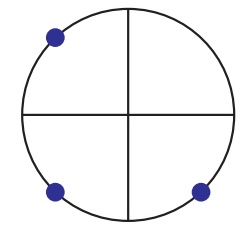

(59)

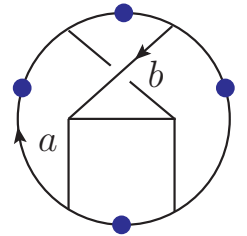

(64)

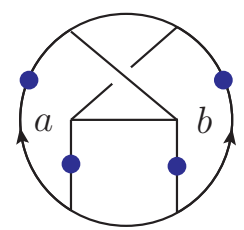

(69)

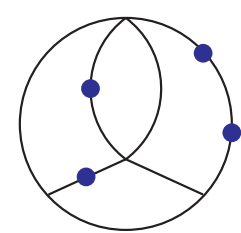

(55)

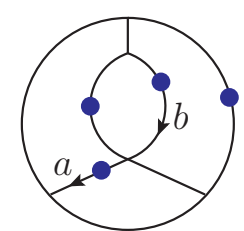

(60)

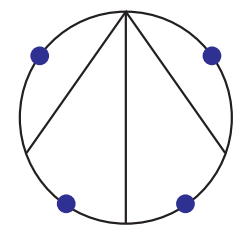

(65)

FIG. 20: Vacuum graphs $I_{51}^{v}$ through $I_{69}^{v}$.

square, $\left(l_{i} \cdot l_{j}\right)^{2}$ for different $i$ and $j$.

To illustrate this procedure in more detail, we consider two examples - the reduction of integrals $I_{66}$ and $I_{12}$. As we will see, the former integral reduces to only scalar integrals, while the latter integral leaves behind a four-tensor integral. The numerator factor of the integral $I_{66}$ in the supergravity amplitude is given by the squaring relation (4.16), and formula (B1) for $N_{66}^{\mathcal{N}=4}$, to be

$$
N_{66}^{\mathcal{N}=8}=s^{2}\left[4 t\left(\tau_{35}-2 \tau_{36}\right)+2 u\left(\tau_{35}+3 \tau_{45}-4 \tau_{46}\right)-s\left(6 u+\tau_{15}-6 t+5 \tau_{25}-8 \tau_{26}\right)\right]^{2} .
$$

For this 12-propagator integral, the leading UV behavior comes from terms that are quadratic in the loop momenta in $N_{66}^{\mathcal{N}=8}$, so the two $\tau$-independent terms inside the brackets in eq. (4.22) may be dropped. For the remaining terms, eq. (4.17) implies that $N_{66}$ is equivalent under integration to

$$
\frac{1}{s} N_{66}^{\mathcal{N}=8} \mapsto \frac{8}{D} s^{2} t u\left(11 \tau_{55}-48 \tau_{56}+48 \tau_{66}\right)-\frac{2}{D} s^{4}\left(15 \tau_{55}-64 \tau_{56}+64 \tau_{66}\right) .
$$

We have extracted a factor of $1 / s$ on the left-hand side from the external propagator in the $1 \mathrm{PR}$ integral $I_{66}$. 
Next we express all scalar products of internal momenta in terms of inverse propagators, using $\tau_{55}=2 l_{5}^{2}$ and $\tau_{66}-\tau_{56}=-l_{5}^{2}+l_{6}^{2}+\left(l_{5}-l_{6}\right)^{2}$. Summing over the 4 ! permutations, and dividing by the symmetry factor of 4 for this graph, we find that the contribution of $I_{66}$ is

$$
\frac{1}{s} N_{66}^{\mathcal{N}=8} \mapsto \frac{4}{D}\left(s^{2}+t^{2}+u^{2}\right)^{2}\left(17 l_{5}^{2}-32\left(l_{5}-l_{6}\right)^{2}-32 l_{6}^{2}\right) .
$$

In the permutation sum the first term in eq. (4.23) cancels out completely due to $s+t+u=0$.

Each one of the factors $l_{5}^{2},\left(l_{5}-l_{6}\right)^{2}$ and $l_{6}^{2}$ in eq. (4.24) cancels one propagator of $I_{66}$. The topology resulting from $l_{5}^{2}$ can be identified as graph (17) in fig. 18. The $\left(l_{5}-l_{6}\right)^{2}$ and $l_{6}^{2}$ terms both lead to graph (1) in fig. 18. Therefore, to leading order in the small external momentum expansion in $D=11 / 2$, the contribution of integral $I_{66}$ to $\widetilde{\mathcal{V}}^{(4)}$ becomes

$$
I_{66} \mapsto \frac{32}{11}\left(17 \widetilde{I}_{17}^{v}-64 \widetilde{I}_{1}^{v}\right)=\frac{544}{11} \widetilde{I}_{17}^{v}-\frac{2048}{11} \widetilde{I}_{1}^{v}
$$

Here the $\widetilde{I}_{i}^{v}$ denote the vacuum integrals with topologies shown in figures 18-20, and momentum-dependent numerator factors shown in the first column of table I, but with the rational numerical coefficients set to unity. For example, with this notation we have $I_{1}^{v}=-\frac{117674}{1485} \widetilde{I}_{1}^{v}$. We have multiplied by $4 /\left(s^{2}+t^{2}+u^{2}\right)^{2}$ in passing from eq. (4.24) to eq. (4.25), to account for the relative prefactor of $\left(s^{2}+t^{2}+u^{2}\right)^{2} / 4$ in eq. (4.20), compared with eq. (3.2). The difference between the numerical coefficients in eq. (4.25) and those in table I is simply that the table collects the contributions to each vacuum graph from all the different integrals $I_{i}$ appearing in eq. (3.2).

As a second example, consider the reduction of $I_{12}$. The numerator factor in the $\mathcal{N}=8$ supergravity amplitude is

$$
\begin{aligned}
N_{12}^{\mathcal{N}=8}= & \frac{s^{2}}{4}\left[s\left(\tau_{16}-\tau_{26}-\tau_{35}+\tau_{45}+2 t\right)\right. \\
& \left.+2 s \tau_{56}-2\left(4 \tau_{16} \tau_{25}+4 \tau_{15} \tau_{26}+\tau_{45}\left(\tau_{36}-3 \tau_{46}\right)+\tau_{35}\left(\tau_{46}-3 \tau_{36}\right)\right)\right]^{2} .
\end{aligned}
$$

The leading UV divergence requires four powers of the loop momenta from the numerator. That can come only from the second and third terms inside the square; the first term is linear in the loop momentum and can be dropped. Using eq. (4.18) and the on-shell condition for the external legs we find, after some straightforward calculation, that under the integral sign $N_{12}^{\mathcal{N}=8}$ is equivalent to

$$
\begin{aligned}
N_{12}^{\mathcal{N}=8} \mapsto & -\frac{128}{(D-1) D(D+2)} s^{2} t u\left[(D-2) \tau_{56}^{2}+D \tau_{55} \tau_{66}\right] \\
& +\frac{1}{(D-1) D(D+2)} s^{4}\left[\left(D^{3}-19 D^{2}+146 D-96\right) \tau_{56}^{2}+4(17 D-25) \tau_{55} \tau_{66}\right] .
\end{aligned}
$$

By inspecting the graph for $I_{12}$ it is straightforward to see that $\tau_{56}$ cannot be completely expressed in terms of inverse propagators. We therefore keep it in this form. The sum over the 4 ! permutations leads, as in the case of $N_{66}^{\mathcal{N}}=8$, to the complete cancellation of the term proportional to $s^{2} t u$. Dividing by the symmetry factor of 4 for $I_{12}$, we find that

$$
N_{12}^{\mathcal{N}=8} \mapsto\left(s^{2}+t^{2}+u^{2}\right)^{2}\left[\frac{D^{3}-19 D^{2}+146 D-96}{(D-1) D(D+2)} \tau_{56}^{2}+16 \frac{17 D-25}{(D-1) D(D+2)} l_{5}^{2} l_{6}^{2}\right] .
$$


Considering the graph associated with $I_{12}$, we see that the $\tau_{56}^{2}$ term generates graph (3) in fig. 18, whereas the factor of $l_{5}^{2} l_{6}^{2}$ reduces the tripled propagators to doubled ones, generating graph (4) in fig. 18. Multiplying by an overall factor of $4 /\left(s^{2}+t^{2}+u^{2}\right)^{2}$ and setting $D=11 / 2$, we obtain for the contribution of $I_{12}$ to $\widetilde{\mathcal{V}}^{(4)}$,

$$
I_{12} \mapsto \frac{9556}{1485} \widetilde{I}_{3}^{v}+\frac{35072}{1485} \widetilde{I}_{4}^{v}
$$

It turns out that integral $I_{12}$ generates the only contribution to $I_{3}^{v}$, so that $I_{3}^{v}=\frac{9556}{1485} \widetilde{I}_{3}^{v}$, as given in table $\mathrm{I}$ in appendix C. Several other integrals $I_{i}$ contribute to $\widetilde{I}_{4}^{v}$, so the rational coefficient given in table I for $I_{4}^{v}$ is different from the one in eq. (4.29).

Carrying out the steps detailed above for all the integrals appearing in the amplitude, we find that they reduce to 92 different vacuum integral topologies. In the complete amplitude some of them cancel out ${ }^{5}$, leaving only the 69 vacuum topologies shown in figures 18-20. While these integrals may be rearranged somewhat using momentum conservation, to find the full set of relations between integrals we need more powerful methods. We shall see in the next section that integration-by-parts identities reduce them all to linear combinations of the three scalar vacuum integrals shown in fig. 17.

\section{Integrating the vacuum graphs}

To evaluate vacuum integrals in their critical dimension $D_{c}=11 / 2$, we use the same infrared rearrangement [67] (related to the $R^{*}$ operation [75]) that we used previously to evaluate the three-loop vacuum integrals $V^{(\mathrm{A})}$ and $V^{(\mathrm{B})}[55]$ and the four-loop vacuum integrals $V_{1}, V_{2}$ and $V_{8}$ [11]. This approach was already discussed in some detail in ref. [11], so here we include only a brief summary.

The expansion in small external momentum introduces unphysical infrared divergences. To separate them from the UV singularities we inject and remove momentum $k_{\mu}$, with $k^{2} \neq 0$, at two of the vertices of the vacuum integral, thus transforming it into a four-loop two-point integral. This two-point integral possesses the same UV poles as the vacuum integral, but no infrared divergences. We always take the two vertices in question to be connected by a single propagator in the four-loop vacuum integral. In this case, the four-loop two-point integrals can be factorized into products of three-loop two-point integrals and one-loop two-point integrals. The one-loop two-point integrals are trivial, and contain the UV 1/ $\epsilon$ pole. The finite three-loop two-point propagator integrals are then evaluated through the method of integration by parts (IBP) [76], implemented in the MINCER algorithm. For certain tensor integrals we also applied the Laporta algorithm [60] for solving the integration-by-parts relations, as implemented in the computer code AIR [77]. In addition, we employed gluing relations [76], which demand consistency of the various ways of factorizing the four-loop UV-divergent integral into products of lower-loop integrals. These consistency conditions are nontrivial and aid in the evaluation of some of the master integrals remaining after IBP reduction in $D=11 / 2$.

Further consistency relations between vacuum integrals can be derived as follows. We start with a four-loop two-point integral with a numerator factor of degree less than or equal to two in the loop momenta. We expand it to next-to-next-to-leading order in the

\footnotetext{
${ }^{5}$ Some of these cancellations are dimension-independent, while others occur only in $D=11 / 2$.
} 
small external momenta, using different parametrizations of the loop momenta. We require that the different parametrizations yield consistent results. These relations may be used either to reduce the number of integrals that need to be evaluated, or as consistency checks of the integral evaluation. A similar strategy was used to show that the four-loop fourpoint $\mathcal{N}=8$ supergravity amplitude is UV finite in $D=5$ [10]. In that case, the complete amplitude was expanded for different loop-momentum parametrizations. This approach led to consistency relations involving only integrals that naturally appear in the amplitude. The present situation is somewhat more involved because evaluation of each of the 69 integrals implies that we need to expand integrals which do not necessarily appear in the final result. In this case, the consistency relations that are generated involve a much larger set of integrals than just the 69 under consideration.

Following the strategies reviewed above, all the 69 integrals appearing in the small momentum expansion of the four-loop four-point $\mathcal{N}=8$ supergravity amplitude can be reduced to linear combinations of the integrals $V_{1}, V_{2}$ and $V_{8}$ shown in fig. 17. These integrals have already appeared in the UV divergence of the corresponding $\mathcal{N}=4$ sYM amplitude. The coefficients of $V_{1}, V_{2}$ and $V_{8}$ after this reduction are provided in the second, third and fourth columns, respectively, of table $\mathrm{I}$ in appendix $\mathrm{C}$. The values of the $\mathrm{UV}$ poles of $V_{1}, V_{2}$ and $V_{8}$ were determined in ref. [11] and are given in eq. (4.15).

It is interesting to complete the evaluation of the two examples discussed in the previous subsection, $I_{66}$ and $I_{12}$, and thereby illustrate a general feature regarding the positivity properties of the residues of the UV poles of the integrals appearing in the $\mathcal{N}=8$ supergravity amplitude in the double-copy representation.

The results of appendix $\mathrm{C}$ can be used (after dividing by rational coefficients in the second column) to express the following integrals $\widetilde{I}_{i}^{v}$ in terms of the basis integrals $V_{1}, V_{2}$ and $V_{8}$ :

$$
\begin{aligned}
\widetilde{I}_{1}^{v} & =V_{2}, \\
\widetilde{I}_{3}^{v} & =\frac{6671}{4800} V_{1}-\frac{531}{800} V_{2}, \\
\widetilde{I}_{4}^{v} & =V_{1}, \\
\widetilde{I}_{17}^{v} & =\frac{3}{4} V_{1}+\frac{1}{2} V_{2},
\end{aligned}
$$

Inserting these equations into eq. (4.25), for the contribution of $I_{66}$ to $\widetilde{\mathcal{V}}^{(4)}$ we obtain

$$
I_{66} \mapsto \frac{544}{11} \widetilde{I}_{17}^{v}-\frac{2048}{11} \widetilde{I}_{1}^{v}=\frac{408}{11} V_{1}-\frac{1776}{11} V_{2}
$$

Using the expressions (4.15) for $V_{1}$ and $V_{2}$ we find that the sign of the UV divergence of $I_{66}$ is negative.

Similarly, the contribution of the UV singularity of $I_{12}$ to $\widetilde{\mathcal{V}}^{(4)}$ is

$$
I_{12} \mapsto \frac{9556}{1485} \widetilde{I}_{3}^{v}+\frac{35072}{1485} \widetilde{I}_{4}^{v}=\frac{58023419}{1782000} V_{1}-\frac{140951}{33000} V_{2}
$$

Inserting the numerical values of $V_{1}$ and $V_{2}$ into eq. (4.35) we find that, unlike $I_{66}$, the UV pole of $I_{12}$ has a positive residue in $D=11 / 2$.

We see that the residue of the leading UV pole can be either positive or negative, despite the fact that the initial numerator factors are perfect squares. This phenomenon occurs frequently in the reduction of the various integrals appearing in the $\mathcal{N}=8$ supergravity 
amplitudes. It leads to strong cancellations among the corresponding UV poles. The origin of this phenomenon is simply that the propagators occurring in the denominators of the integrand do not have a fixed sign. For example, the $1 \mathrm{PR}$ integral $I_{66}$ contains an explicit factor of $1 / s$ from a propagator external to the loops. The sum over its permutations contains factors of $1 / t$ and $1 / u$. Not all of these factors can be positive, given that $s+t+u=0$. Even if these factors are multiplied by positive numbers, the sum can be negative. Indeed, in any physical region (with one Mandelstam invariant positive and two negative), if they were all multiplied by the same number, the sum would be negative, because $\frac{1}{s}+\frac{1}{t}+\frac{1}{u}<0$ for $s+t+u=0$.

\section{The $\mathcal{N}=8$ supergravity UV behavior}

The expressions for the leading UV divergences of the 69 vacuum integrals $I_{i}^{v}$ in $D=$ $11 / 2-2 \epsilon$ are collected in table I in appendix C. Totaling up these contributions, we determine the value of $\widetilde{\mathcal{V}}^{(4)}$, defined through eqs. (4.20) and (4.21), to be

$$
\widetilde{\mathcal{V}}^{(4)}=\frac{23}{2}\left(V_{1}+2 V_{2}+V_{8}\right) \text {. }
$$

Plugging this value for $\widetilde{\mathcal{V}}^{(4)}$ into eq. (4.20), the UV singularity of the four-loop four-point $\mathcal{N}=8$ supergravity amplitude is given by

$$
\left.\mathcal{M}_{4}^{(4)}\right|_{\text {pole }}=-\frac{23}{8}\left(\frac{\kappa}{2}\right)^{10} s t u\left(s^{2}+t^{2}+u^{2}\right)^{2} M_{4}^{\text {tree }}\left(V_{1}+2 V_{2}+V_{8}\right) .
$$

Because this result is nonvanishing, the four-loop four-point amplitude of $\mathcal{N}=8$ supergravity diverges in exactly the same critical dimension $D_{c}=11 / 2$ as that for $\mathcal{N}=4$ sYM theory.

Comparing eqs. (4.37) and (4.14), we see that the leading UV divergence of the fourloop four-point $\mathcal{N}=8$ supergravity amplitude is given by the same combination of vacuum integrals as the $1 / N_{c}^{2}$-suppressed single-trace UV divergence of the four-point $\mathcal{N}=4 \mathrm{sYM}$ amplitude, namely $V_{1}+2 V_{2}+V_{8}$. This pattern matches our observations above regarding the two- and three-loop amplitudes. While we do not understand the full significance of this close relation between the UV divergences of the gravity and subleading-color sYM amplitudes, it seems unlikely to be accidental. If it persists at higher loops as well, it could potentially have interesting consequences for the higher-loop UV behavior of $\mathcal{N}=8$ supergravity, especially given that $\mathcal{N}=4 \mathrm{sYM}$ is UV finite in four dimensions. The fact that this nontrivial connection holds through at least four loops is a central observation of this paper.

While the divergences of all integrals entering $\mathcal{M}_{4}^{(4)}$ are expressed in terms of the vacuum master integrals $V_{1}, V_{2}$ and $V_{8}$, the expression (4.37) arises as result of extensive cancellations between the various integrals. For example, integrals $I_{80}$ through $I_{85}$ are responsible for the complete UV divergence of the four-loop four-point $\mathcal{N}=4 \mathrm{sYM}$ amplitude. It is easy to see that they contribute to the UV divergence of the $\mathcal{N}=8$ supergravity amplitude the following amount:

$$
\left.\mathcal{M}_{4}^{(4)}\right|_{\text {pole }} ^{I_{80,81,82}}=-64\left(\frac{\kappa}{2}\right)^{10} s t u\left(s^{2}+t^{2}+u^{2}\right)^{2} M_{4}^{\text {tree }}\left(V_{1}+2 V_{2}+V_{8}\right) .
$$

Note that the snail integrals $I_{83}, I_{84}$ and $I_{85}$ yield vanishing contributions in the supergravity case. Because the rational coefficient in eq. (4.37), 23/8, is smaller than 64 , it follows that 


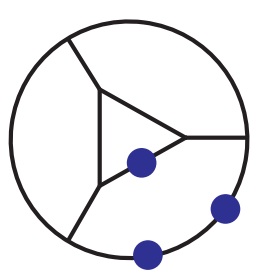

$U_{1}$

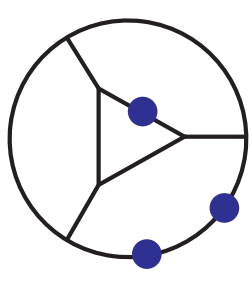

$U_{2}$

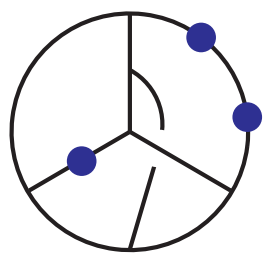

$U_{3}$

FIG. 21: The vacuum integrals $U_{1}, U_{2}$ and $U_{3}$ that would be generated by the graphs 51,52 and 72 in the double-trace part of the four-loop divergence of $\mathcal{N}=4 \mathrm{sYM}$ theory in $D=6$. Because these three graphs possess both an overall divergence and a three-loop subdivergence, we only evaluate them after first performing a subtraction of the subdivergence.

the integrals $I_{1}$ through $I_{79}$ contribute to the UV pole with opposite sign, relative to $I_{80}, I_{81}$ and $I_{82}$. This fact is not surprising: although the numerators are squares, the propagators do not have all the same signs.

To expose a more dramatic numerical cancellation, we have split the above computation into two pieces: The 12-propagator contributions, defined to be those from integrals $I_{53}$ through $I_{79}$, except $I_{72}$, which all have explicit single inverse powers of the Mandelstam variables, $s, t$ or $u$; and the remaining 11- and 13-propagator contributions, which have either no such explicit factor, or else the factor is squared. We find that

$$
\begin{gathered}
\left.\mathcal{M}_{4}^{(4)}\right|_{\text {pole }} ^{12 \text {-propagator }}=+142\left(\frac{\kappa}{2}\right)^{10} s t u\left(s^{2}+t^{2}+u^{2}\right)^{2} M_{4}^{\text {tree }}\left(V_{1}+2 V_{2}+V_{8}\right), \\
\left.\mathcal{M}_{4}^{(4)}\right|_{\text {pole }} ^{11,13 \text {-propagator }}=-\frac{1159}{8}\left(\frac{\kappa}{2}\right)^{10} s t u\left(s^{2}+t^{2}+u^{2}\right)^{2} M_{4}^{\text {tree }}\left(V_{1}+2 V_{2}+V_{8}\right) .
\end{gathered}
$$

Remarkably, the numerical cancellation between these two sets of contributions, in order to get the total (4.37), is quite significant, to within about $2 \%$. That is, $(23 / 8) / 142=0.0202 \ldots$

In summary, by carrying out the integration to extract the four-loop four-point UV divergence of $\mathcal{N}=8$ supergravity in $D=11 / 2$, we have found extensive cancellations, both analytical and numerical, once again revealing surprising hidden structure.

\section{E. The $\mathcal{N}=4 \mathrm{sYM}$ color double-trace UV divergence}

In ref. [11] we showed explicitly that the color double-trace terms in the four-point $\mathcal{N}=4$ sYM amplitudes are better-behaved in the UV than the single-trace terms, starting at three loops. In particular, they obey the finiteness bound $D \leq 4+8 / L$ at three and four loops. This improved behavior has been discussed from the vantage points of both string theory and field theory $[64,69]$. The form of the four-loop four-point sYM amplitude constructed here allows us to evaluate the four-loop double-trace divergence in $D=6-2 \epsilon$ dimensions and probe whether the bound is saturated. We will find that the double-trace term does indeed diverge, and so the bound is saturated. A subtlety arises in this calculation because the single-trace terms in the three-loop four-point amplitude diverge in six dimensions. Therefore the extraction of the four-loop four-point double-trace divergence requires a careful subtraction of the contribution of the three-loop counterterm.

In light of the presence of subdivergences in $D=6-2 \epsilon$, it is useful to separate the integrals appearing in the four-loop amplitude into those that cannot have subdivergences 

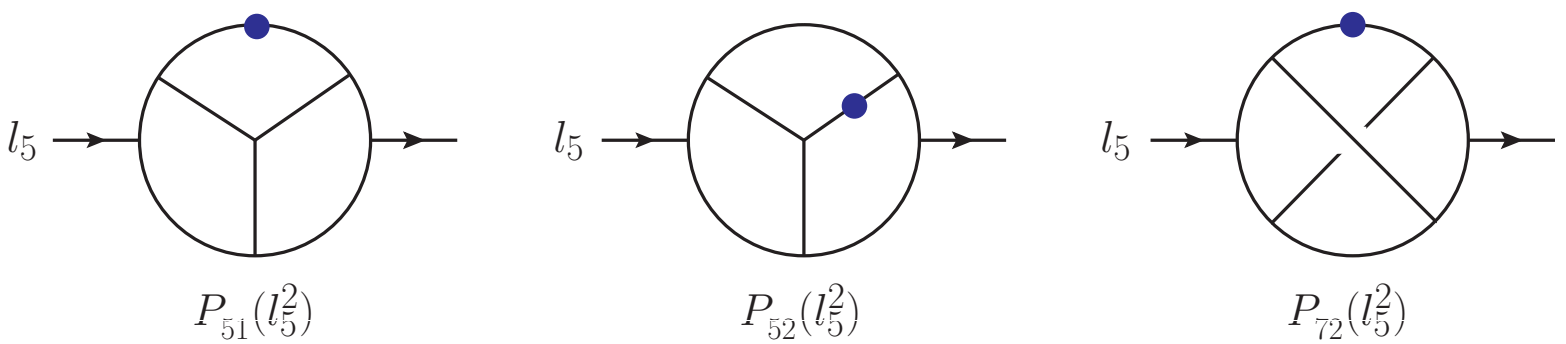

FIG. 22: Three-loop propagator subgraphs $P_{51}\left(l_{5}^{2}\right), P_{52}\left(l_{5}^{2}\right)$ and $P_{72}\left(l_{5}^{2}\right)$, generated by setting $k_{4} \rightarrow 0$ in graphs 51, 52 and 72 , respectively.

(graphs 1 through 50) and those that might (graphs 51 through 85). The extraction of UV divergences uses precisely the same methods as discussed above in the analysis of the UV behavior of the $\mathcal{N}=8$ supergravity amplitude. For the first set of graphs, we simply quote the result without providing further details. The UV divergence from graphs 1 through 50 has the following $\operatorname{Tr}_{12} \operatorname{Tr}_{34}$ component:

$$
\begin{aligned}
\left.\mathcal{A}_{4}^{(4)}\right|_{\text {pole 1-50 }} ^{\operatorname{Tr}_{12} \operatorname{Tr}_{34}}= & \frac{g^{10} \mathcal{K}}{3(4 \pi)^{12} \epsilon} \operatorname{Tr}_{12} \operatorname{Tr}_{34} N_{c} \\
& \times\left[\left(s^{2}+t^{2}+u^{2}\right)\left(N_{c}^{2}\left(1-4 \zeta_{3}+10 \zeta_{5}\right)+180 \zeta_{5}\right)-9 s^{2}\left(N_{c}^{2} \zeta_{3}+25 \zeta_{5}\right)\right]
\end{aligned}
$$

where $\mathcal{K}$ is defined in eq. (4.4). The other double-trace components can be obtained by permuting the external momenta in this expression. The absence of a $1 / \epsilon^{2}$ pole signals that this class of graphs indeed has no subdivergences.

Next we evaluate the remaining graphs, 51 through 85 . Because the color factors of $1 \mathrm{PR}$ graphs do not contain double traces, it follows that, in fact, only the integrals $I_{51}, I_{52}$ and $I_{72}$ contribute to the double-trace terms. If they did not have subdivergences, we could evaluate them directly by setting the external momenta to zero, leading to the vacuum integrals $U_{1}$, $U_{2}$ and $U_{3}$ shown in fig. 21. Instead, we will perform a subtraction of the subdivergence and evaluate the inner three-loop integral first, before evaluating the integral over the outer loop momentum.

We first note from eq. (A5) that the numerator factors of these three integrals are all equal, $N_{51}=N_{52}=N_{72}$. All three integrals contain an essentially identical subdivergence, from a three-point three-loop subgraph whose external legs carry momentum $k_{4}, l_{5}$ and $l_{5}+k_{4}$. In the evaluation of this inner graph, and its subtraction term, if we are only interested in the final $1 / \epsilon^{2}$ and $1 / \epsilon$ contributions, we can neglect the dependence on $k_{4}$ : the $1 / \epsilon^{2}$ divergence is independent of the details of the external momenta, and the $1 / \epsilon$ contribution, arising from the finite part of the three-loop subgraph, comes from the integration region $l_{5} \gg k_{4}$ where the outer loop diverges. Then the three-point subgraph reduces to a propagator (two-point) subgraph, as shown in fig. 22 for the respective cases of graphs 51, 52 and 72 . The blue dot indicates the location of the doubled propagator that is generated in the limit $k_{4} \rightarrow 0$.

These integrals can be evaluated in $D=6-2 \epsilon$ using IBP identities and gluing relations 
through the necessary order, $\mathcal{O}\left(\epsilon^{0}\right)$. The results are:

$$
\begin{aligned}
& P_{51}\left(l_{5}^{2}\right)=-\left(-l_{5}^{2}\right)^{-3 \epsilon} \frac{e^{-3 \gamma \epsilon}}{(4 \pi)^{9-3 \epsilon}}\left[\frac{1}{6 \epsilon}+\frac{25}{9}+\zeta_{3}-\frac{10}{3} \zeta_{5}+\mathcal{O}(\epsilon)\right], \\
& P_{52}\left(l_{5}^{2}\right)=-\left(-l_{5}^{2}\right)^{-3 \epsilon} \frac{e^{-3 \gamma \epsilon}}{(4 \pi)^{9-3 \epsilon}}\left[\frac{1}{6 \epsilon}\left(\zeta_{3}-\frac{1}{3}\right)-\frac{25}{27}+\frac{17}{18} \zeta_{3}+\frac{1}{4} \zeta_{4}+\mathcal{O}(\epsilon)\right], \\
& P_{72}\left(l_{5}^{2}\right)=-\left(-l_{5}^{2}\right)^{-3 \epsilon} \frac{e^{-3 \gamma \epsilon}}{(4 \pi)^{9-3 \epsilon}}\left[\frac{1}{6 \epsilon}\left(\zeta_{3}-\frac{1}{3}\right)-\frac{25}{27}+\frac{17}{18} \zeta_{3}+\frac{1}{4} \zeta_{4}+\mathcal{O}(\epsilon)\right] .
\end{aligned}
$$

We note that the propagator subgraphs for graphs 52 and 72 happen to have identical values through $\mathcal{O}(\epsilon)$. Also, inspecting the form of the integrals, and comparing with eqs. (4.10) and (4.11), we see that the leading $1 / \epsilon$ singularities correspond to the three-loop vacuum integrals $V^{(\mathrm{A})}$ (for the planar graph 51) and $V^{(\mathrm{B})}$ (for graphs 52 and 72 ).

In the trace basis, the color factors for graphs 51, 52 and 72 have the following form:

$$
\begin{aligned}
& C_{51}=N_{c}^{2}\left(N_{c}^{2}+12\right)\left(\operatorname{Tr}_{1234}+\operatorname{Tr}_{1432}\right) \\
&+2 N_{c}\left(N_{c}^{2}+12\right)\left(\operatorname{Tr}_{12} \operatorname{Tr}_{34}+\operatorname{Tr}_{13} \operatorname{Tr}_{24}+\operatorname{Tr}_{14} \operatorname{Tr}_{23}\right) \\
& C_{52}=C_{72}=12 N_{c}^{2}\left(\operatorname{Tr}_{1234}+\operatorname{Tr}_{1432}\right)+24 N_{c}\left(\operatorname{Tr}_{12} \operatorname{Tr}_{34}+\operatorname{Tr}_{13} \operatorname{Tr}_{24}+\operatorname{Tr}_{14} \operatorname{Tr}_{23}\right) .
\end{aligned}
$$

The double-trace parts of these color factors contain a piece proportional to $N_{c}^{3}$ and one proportional to $N_{c}^{1}$. The $N_{c}^{3}$ part comes only from graph 51. The $N_{c}^{1}$ parts of these color factors are equal for the three graphs. Taking into account the relative symmetry factors in eq. (3.3), we see that the relevant linear combination of propagator integrals for the $N_{c}^{1}$ part is $P_{N_{c}^{1}} \equiv P_{51}+2 P_{52}+P_{72}$, which is given by,

$$
P_{N_{c}^{1}}\left(l_{5}^{2}\right)=-\left(-l_{5}^{2}\right)^{-3 \epsilon} \frac{e^{-3 \gamma \epsilon}}{(4 \pi)^{9-3 \epsilon}}\left[\frac{\zeta_{3}}{2 \epsilon}+\frac{23}{6} \zeta_{3}+\frac{3}{4} \zeta_{4}-\frac{10}{3} \zeta_{5}+\mathcal{O}(\epsilon)\right] .
$$

Next we need to identify a subtraction that accounts for the three-loop counterterm needed to cancel the pole given in eq. (4.9). We could compute the four-loop counteramplitude by sewing a tree amplitude onto the matrix element of the counterterm (essentially the negative of eq. (4.9)), in close analogy to how we evaluated the UV divergence of the three-loop four-point supergravity amplitude in odd dimensions above $D=6$ [55]. However, it is safer to perform the subtraction within the integrals for the individual graphs 51,52 and 72 , in order to ensure that there are no spurious contributions arising from potentially different ways of regularizing the infrared behavior.

In discussing higher-loop divergences of a theory, there is always a freedom associated with additional, finite renormalizations of the theory at lower loops, in this case three loops. Here we will choose, for definiteness and simplicity, an $\overline{\mathrm{MS}}$ scheme for the three-loop renormalization. In this scheme, the necessary counterterms are

$$
\begin{aligned}
& P_{51}^{\text {c.t. }}=\frac{e^{-3 \gamma \epsilon}}{(4 \pi)^{9-3 \epsilon}} \frac{1}{6 \epsilon}, \\
& P_{N_{c}^{1}}^{\text {c.t. }}=\frac{e^{-3 \gamma \epsilon}}{(4 \pi)^{9-3 \epsilon}} \frac{\zeta_{3}}{2 \epsilon} .
\end{aligned}
$$

Notice that the factor of $\left(-l_{5}^{2}\right)^{-3 \epsilon}$ in eqs. (4.42) and (4.47) is absent in the counterterm contributions (4.48) and (4.49). 
The evaluations of the remaining one-loop integrals and of their subtraction are essentially identical for the graphs 51, 52 and 72 . We will discuss in detail only the graph 51 for which we consider the following subtracted integral,

$$
I_{51}^{\mathrm{sub}} \equiv-i \int \frac{d^{6-2 \epsilon} l_{5}}{(2 \pi)^{6-2 \epsilon}} \frac{N_{51}\left(l_{5}\right)}{l_{5}^{2}\left(l_{5}-k_{1}\right)^{2}\left(l_{5}-k_{12}\right)^{2}\left(l_{5}-k_{123}\right)^{2}}\left[P_{51}\left(l_{5}^{2}\right)+P_{51}^{\text {c.t. }}\right] .
$$

If a term in the outer integral over $l_{5}$ is UV convergent, one can set $\epsilon \rightarrow 0$ and the $1 / \epsilon$ poles will cancel in the brackets, leaving no UV contribution at all at four loops. On the other hand, if a term in the outer integral generates a logarithmic divergence, as in the terms in $N_{51}$ that are quadratic in $l_{5}$, then the leading $1 / \epsilon^{2}$ pole from $P_{51}\left(l_{5}^{2}\right)$ is not cancelled by the counterterm contribution $P_{51}^{\text {c.t. }}$. Accounting for a factor of $\left(-l_{5}^{2}\right)^{-\epsilon}$ from the one-loop integration measure, we see that there is a mismatch by a factor of four. That is, the leading $1 / \epsilon^{2}$ contribution from $P_{51}^{\text {c.t. }}$ is of opposite sign to that from $P_{51}\left(l_{5}^{2}\right)$ and is four times larger in magnitude, due to a factor of $\left(-l_{5}^{2}\right)^{-\epsilon}$ instead of $\left(-l_{5}^{2}\right)^{-4 \epsilon}$.

The relative factor of four originates physically from the locality of the UV poles in the subtracted four-loop amplitude. The four-loop amplitude carries fractional dimension $\propto s^{-4 \epsilon}$, while the one-loop counterterm amplitude carries fractional dimension $\propto s^{-\epsilon}$. Expanding these $s$-dependent factors in $\epsilon$, we see that a ratio of $(-4)$ between the $1 / \epsilon^{2}$ poles from the amplitude and its counter-amplitude is required, in order for non-local terms of the form $1 / \epsilon \times \ln \left(-s_{i j}\right)$ to cancel in the subtracted amplitude.

From eq. (B1), the quadratic terms in the numerator factor $N_{51}$ are given by,

$$
\begin{aligned}
N_{51}^{\text {quad }}=\frac{1}{2}[ & -6\left(t \tau_{15}^{2}+u \tau_{25}^{2}+s \tau_{35}^{2}\right)+5\left(s \tau_{15} \tau_{25}+t \tau_{25} \tau_{35}+u \tau_{15} \tau_{35}\right) \\
& \left.-\left(s^{2}+t^{2}+u^{2}\right) l_{5}^{2}\right] .
\end{aligned}
$$

We insert eq. (4.51) into eq. (4.50) and Feynman parametrize the resulting one-loop integral. As usual with a one-loop integral with numerator quadratic in the loop momentum, there are two types of terms:

1. terms that depend in a fairly complicated way on the Feynman parameters and external momentum invariants, originating from the shift $l_{5}^{\mu}=q^{\mu}+\Delta^{\mu}$ in the loop momentum $l_{5}$ needed to complete the square in the denominator, and

2. terms from integrating over the shifted loop momentum $q^{\mu}$, in which one can use the identity $q^{\mu} q^{\nu}=q^{2} / D \times \eta^{\mu \nu}$.

The former terms do not contain an ultraviolet divergence from the outer integral; therefore they can be dropped as discussed above. In the latter terms, the first set of terms in $N_{51}^{\text {quad }}$ in eq. (4.51), containing the -6 prefactor, drop out because $k_{1}^{2}=k_{2}^{2}=k_{3}^{2}=0$. In the second set of terms in $N_{51}^{\text {quad }}$, one can effectively make the replacement (4.17), because any extra terms due to external momentum dependence are finite and unrelated to the UV divergence and therefore drop out. (We checked this statement by performing a full Feynman parametrization.)

Therefore, the $1 / \epsilon^{2}$ and $1 / \epsilon$ terms in $I_{51}^{\text {sub }}$ in eq. (4.50) are correctly captured by

$$
I_{51}^{\text {sub }}=-i\left(\frac{5}{D}-\frac{1}{2}\right)\left(s^{2}+t^{2}+u^{2}\right) \int \frac{d^{D} l_{5}}{(2 \pi)^{D}} \frac{P_{51}\left(l_{5}^{2}\right)+P_{51}^{\text {c.t. }}}{\left(l_{5}-k_{1}\right)^{2}\left(l_{5}-k_{12}\right)^{2}\left(l_{5}-k_{123}\right)^{2}}+\mathcal{O}\left(\epsilon^{0}\right) .
$$


We do not need to evaluate this full integral; to extract the UV pole it is sufficient to simplify it to the form of a massive bubble integral by rearranging the external momenta,

$$
I_{51}^{\text {sub }}=-i\left(\frac{5}{D}-\frac{1}{2}\right)\left(s^{2}+t^{2}+u^{2}\right) \int \frac{d^{D} l_{5}}{(2 \pi)^{D}} \frac{P_{51}\left(l_{5}^{2}\right)+P_{51}^{\text {c.t. }}}{\left(l_{5}^{2}\right)^{2}\left(l_{5}-k_{12}\right)^{2}}+\mathcal{O}\left(\epsilon^{0}\right) .
$$

In the term coming from $P_{51}\left(l_{5}^{2}\right)$ in eq. (4.53), the powers to which the two propagators in the $D=6-2 \epsilon$ bubble (after analytic continuation) are raised are $2+3 \epsilon$ and 1 ; while in the term coming from $P_{51}^{\text {c.t. }}$ they are 2 and 1 .

The momentum-independent parts of these bubble integrals are given by

$$
\begin{aligned}
P^{(1)}(2+3 \epsilon, 1 ; 6-2 \epsilon) & =-\frac{e^{-\gamma \epsilon}}{(4 \pi)^{3-\epsilon}}\left[\frac{1}{8 \epsilon}+\frac{7}{16}+\mathcal{O}(\epsilon)\right], \\
P^{(1)}(2,1 ; 6-2 \epsilon) & =-\frac{e^{-\gamma \epsilon}}{(4 \pi)^{3-\epsilon}}\left[\frac{1}{2 \epsilon}+1+\mathcal{O}(\epsilon)\right] .
\end{aligned}
$$

(Other ways of regulating the infrared behavior, such as keeping the complete dependence on external momenta, will give different $\mathcal{O}\left(\epsilon^{0}\right)$ terms in eqs. (4.54) and (4.55). However, the difference must always be the same; otherwise a $1 / \epsilon$ UV pole would be generated from a UV convergent integral.) Including the overall factors, we get,

$$
\begin{aligned}
I_{51}^{\text {sub }}= & \left(\frac{5}{6-2 \epsilon}-\frac{1}{2}\right) \frac{e^{-4 \gamma \epsilon}}{(4 \pi)^{12-4 \epsilon}}\left(s^{2}+t^{2}+u^{2}\right) \\
& \times\left\{\left[\frac{1}{6 \epsilon}+\frac{25}{9}+\zeta_{3}-\frac{10}{3} \zeta_{5}\right]\left(\frac{1}{8 \epsilon}+\frac{7}{16}\right)-\frac{1}{6 \epsilon}\left(\frac{1}{2 \epsilon}+1\right)+\mathcal{O}\left(\epsilon^{0}\right)\right\} \\
= & \frac{e^{-4 \gamma \epsilon}}{(4 \pi)^{12-4 \epsilon}} \frac{s^{2}+t^{2}+u^{2}}{24}\left[-\frac{1}{2 \epsilon^{2}}+\frac{1}{\epsilon}\left(\frac{29}{18}+\zeta_{3}-\frac{10}{3} \zeta_{5}\right)\right]+\mathcal{O}\left(\epsilon^{0}\right) .
\end{aligned}
$$

Similarly, the $N_{c}^{1}$ double-trace contribution is obtained using the same formula (4.53) with $P_{51}$ replaced by $P_{N_{c}^{1}}$, taken from eqs. (4.47) and (4.49),

$$
\begin{aligned}
I_{N_{c}^{1}}^{\mathrm{sub}}= & \left(\frac{5}{6-2 \epsilon}-\frac{1}{2}\right) \frac{e^{-4 \gamma \epsilon}}{(4 \pi)^{12-4 \epsilon}}\left(s^{2}+t^{2}+u^{2}\right) \\
& \times\left\{\left[\frac{\zeta_{3}}{2 \epsilon}+\frac{23}{6} \zeta_{3}+\frac{3}{4} \zeta_{4}-\frac{10}{3} \zeta_{5}\right]\left(\frac{1}{8 \epsilon}+\frac{7}{16}\right)-\frac{\zeta_{3}}{2 \epsilon}\left(\frac{1}{2 \epsilon}+1\right)+\mathcal{O}\left(\epsilon^{0}\right)\right\} \\
= & \frac{e^{-4 \gamma \epsilon}}{(4 \pi)^{12-4 \epsilon}} \frac{s^{2}+t^{2}+u^{2}}{24}\left[-\frac{3 \zeta_{3}}{2 \epsilon^{2}}+\frac{1}{\epsilon}\left(\frac{1}{3} \zeta_{3}+\frac{3}{4} \zeta_{4}-\frac{10}{3} \zeta_{5}\right)\right]+\mathcal{O}\left(\epsilon^{0}\right) .
\end{aligned}
$$

We notice that, similarly to the numerator factor $N_{51}^{\text {quad }}$ in eq. (4.51), both eqs. (4.56) and (4.57) have manifest permutation symmetry.

Plugging eqs. (4.56) and (4.57) into the full amplitude, including the double-trace part of the color factors, the sum over all 24 permutations, and the overall prefactor, we obtain,

$$
\begin{aligned}
\left.\mathcal{A}_{4}^{(4)}\right|_{\text {pole 51-85 }} ^{\text {sub }}= & \frac{g^{10} \mathcal{K} e^{-4 \gamma \epsilon}}{(4 \pi)^{12-4 \epsilon}} N_{c}\left(\operatorname{Tr}_{12} \operatorname{Tr}_{34}+\operatorname{Tr}_{13} \operatorname{Tr}_{24}+\operatorname{Tr}_{14} \operatorname{Tr}_{23}\right)\left(s^{2}+t^{2}+u^{2}\right) \\
& \times\left\{-\frac{N_{c}^{2}+36 \zeta_{3}}{2 \epsilon^{2}}+\frac{1}{\epsilon}\left[N_{c}^{2}\left(\frac{29}{18}+\zeta_{3}-\frac{10}{3} \zeta_{5}\right)+4 \zeta_{3}+9 \zeta_{4}-40 \zeta_{5}\right]\right\} .
\end{aligned}
$$


We have also evaluated the difference between the one-loop outer integrals of $I_{51}, I_{52}$ and $I_{72}$ and their corresponding $\overline{\mathrm{MS}}$ subtraction terms, $I_{51}^{\text {sub }}, I_{52}^{\text {sub }}$ and $I_{72}^{\text {sub }}$, prior to reduction to bubble integrals. That is, we directly integrated eq. (4.50) for general external momenta and separately the outer integral of $I_{51}$ and its subtraction for two special kinematic points. In this way we verified the required cancellation of the nonlocal divergent terms $\ln \left(-s_{i j}\right) / \epsilon$.

Finally, we add the contribution (4.41) from the graphs 1-50 that have no subdivergences, in order to obtain the total four-loop divergence (after subtraction of three-loop subdivergences):

$$
\begin{aligned}
\left.\mathcal{A}_{4}^{(4)}\right|_{\text {pole }} ^{\text {double trace }}= & \left.\mathcal{A}_{4}^{(4)}\right|_{\text {pole 1-50 }} ^{\text {double trace }}+\left.\mathcal{A}_{4}^{(4)}\right|_{\text {pole 51-85 }} ^{\text {sub }} \\
= & \frac{g^{10} \mathcal{K} e^{-4 \gamma \epsilon}}{(4 \pi)^{12-4 \epsilon}} N_{c}\left\{\left(\operatorname{Tr}_{12} \operatorname{Tr}_{34}+\operatorname{Tr}_{14} \operatorname{Tr}_{23}+\operatorname{Tr}_{13} \operatorname{Tr}_{24}\right)\left(s^{2}+t^{2}+u^{2}\right)\right. \\
& \times\left[-\frac{N_{c}^{2}+36 \zeta_{3}}{2 \epsilon^{2}}+\frac{1}{\epsilon}\left(N_{c}^{2}\left(\frac{35}{18}-\frac{\zeta_{3}}{3}\right)+4 \zeta_{3}+9 \zeta_{4}+20 \zeta_{5}\right)\right] \\
& \left.-\frac{3}{\epsilon}\left(N_{c}^{2} \zeta_{3}+25 \zeta_{5}\right)\left(\operatorname{Tr}_{12} \operatorname{Tr}_{34} s^{2}+\operatorname{Tr}_{14} \operatorname{Tr}_{23} t^{2}+\operatorname{Tr}_{13} \operatorname{Tr}_{24} u^{2}\right)\right\}
\end{aligned}
$$

Of course, the double-trace part of the four-loop counterterm must be chosen to cancel these poles,

$$
\left.\mathcal{A}_{4 ; \text { c.t. }}^{(4)}\right|^{\text {double trace }}=-\left.\mathcal{A}_{4}^{(4)}\right|_{\text {pole }} ^{\text {double trace }}+\mathcal{O}(1),
$$

corresponding to a nonvanishing divergent coefficient for a counterterm of the schematic form, $\operatorname{Tr}\left(\mathcal{D}^{4-k} F^{2}\right) \operatorname{Tr}\left(\mathcal{D}^{k} F^{2}\right)$. (The covariant derivatives may be distributed among the two traces in various ways.) It is possible to perform finite shifts of the coefficient of the singletrace operator $\operatorname{Tr}\left(\mathcal{D}^{2} F^{4}\right)$ which has a divergent coefficient at three loops. One can trace through the effect of such a shift by shifting $P_{51}^{\text {c.t. }}$ and $P_{N_{c}^{1}}^{\text {c.t. by }} \mathcal{O}\left(\epsilon^{0}\right)$ constants. In principle, one could remove the $1 / \epsilon$ terms in eq. (4.59) that are proportional to $\left(s^{2}+t^{2}+u^{2}\right)$. However, one cannot remove the $1 / \epsilon^{2}$ pole. Nor can one remove the term proportional to $\left(N_{c}^{2} \zeta_{3}+25 \zeta_{5}\right)$, because the dependence on color and kinematics is different from the one induced by the three-loop counterterm.

In conclusion, the double-trace terms in the four-point $\mathcal{N}=4 \mathrm{sYM}$ amplitude do diverge at four loops, saturating the double-trace finiteness bound of $D_{c}=4+8 / L$.

\section{CONCLUSIONS}

In this paper, we recomputed the four-loop four-point amplitudes of $\mathcal{N}=4$ sYM theory and $\mathcal{N}=8$ supergravity, first obtained in refs. [10,11]. By exploiting the conjectured duality between color and kinematics $[29,30]$ we found greatly simplified representations. It also allowed us to find the form of the complete amplitude, including nonplanar contributions, using only planar cut information as input. We confirmed the correctness of the construction by comparing the unitarity cuts of the new expressions to the cuts of the earlier forms [10, 11]. This provides new nontrivial evidence in favor of the duality conjecture and the associated gravity double-copy property. An important advantage of the current construction is that once the sYM amplitude has been arranged into a duality-satisfying form, the construction 
of the corresponding supergravity integrand is trivial: one simply replaces the color factors with kinematic numerator factors in each graph.

The new form of the four-loop four-point amplitude of $\mathcal{N}=8$ has an important advantage over the previous one [10], because no integral displays a worse UV power counting than the complete amplitude. This feature greatly simplifies the extraction of the UV divergence of the four-loop $\mathcal{N}=8$ supergravity amplitude in the critical dimension $D_{c}=11 / 2$, corresponding to the lowest dimension where both $\mathcal{N}=4$ sYM theory and $\mathcal{N}=8$ supergravity first diverge at four loops. To carry out the required integration we used techniques similar to those described in refs. [9-11]. Our results prove that the four-loop four-point amplitude of $\mathcal{N}=8$ supergravity does indeed diverge in the same critical dimension as the corresponding amplitude of $\mathcal{N}=4$ super-Yang-Mills theory. Thus, the $\mathcal{N}=8$ supergravity finiteness bound [10] is, in fact, saturated at four loops. The amplitude divergence in $D_{c}=11 / 2$ means that the $\mathcal{D}^{8} R^{4}$ supergravity counterterm has a nonzero divergent coefficient, in much the same way as the $\operatorname{Tr}\left(\mathcal{D}^{2} F^{4}\right)$ counterterm of $\mathcal{N}=4$ sYM has a nonvanishing divergent coefficient in this dimension. Moreover, we found that the four-loop finiteness bound [11, 64], $D<6$, for the double-color-trace terms of $\mathcal{N}=4 \mathrm{sYM}$ theory is also saturated. In other words, the corresponding $D=6$ double-trace counterterms $\operatorname{Tr}\left(\mathcal{D}^{4-k} F^{2}\right) \operatorname{Tr}\left(\mathcal{D}^{k} F^{2}\right)$ are also present with non-vanishing coefficients.

More generally, the duality between color and kinematics offers the promise of carrying advances from the planar sector of gauge theory to the nonplanar sector and then to gravity theories. Its underlying origin is, however, still poorly understood; recent progress suggests that, at least in the self-dual case [46], underlying it is an infinite-dimensional Lie algebra of area-preserving diffeomorphisms. Progress has also been made in finding explicit representations of tree amplitudes that manifestly satisfy the duality [47]. It would be interesting and very useful to devise effective rules that would generate directly duality-satisfying representations for loop amplitudes, thus eliminating the need to solve the system of duality constraints on a case-by-case basis. A step towards finding a Lagrangian with the desired properties has been given in ref. [45]. It would also be interesting to explore whether the color-kinematic duality extends beyond weak-coupling perturbation theory as well as whether the existence of such a duality has practical consequences after carrying out the loop-momentum integrations.

Explicit calculations often lead to surprises. The results described here are no different. In particular, in the critical dimension $D=11 / 2$ we found that, after reducing the integrals containing UV divergences to a basis of vacuum integrals encoding the numerical factors in front of the divergent operator, the UV divergence is given by exactly the same combination of basis integrals as found in the single-trace $1 / N_{c}^{2}$-suppressed terms of $\mathcal{N}=4$ sYM theory. It seems unlikely that this is accidental because similar behavior is found at lower loops. It would obviously be important to understand the origin of this curious connection and implications it may have at higher loops on UV divergences. Another interesting property is the existence of strong cancellations between the contributions of various graphs to the UV divergence in the critical dimension. This suggests that different integral contributions may be related to each other by a hidden symmetry.

In summary, the duality between color and kinematics offers a powerful means for streamlining the construction of multiloop amplitudes, carrying advances in the planar sector to the nonplanar sector. It allowed us to express the numerators of the four-loop four-point amplitudes of $\mathcal{N}=4 \mathrm{sYM}$ theory and $\mathcal{N}=8$ supergravity in terms of the numerators of two planar graphs. Using this simplified form, in the critical dimension $D_{c}=11 / 2$, we found 
a surprising coincidence between the UV divergences of $\mathcal{N}=8$ supergravity and those of subleading color single-trace terms of $\mathcal{N}=4 \mathrm{sYM}$ theory. This hints at further new relations between gauge and gravity theories to be unraveled and that further surprises await us at five and higher loops. We look forward to using the tools described in this paper to further explore the multiloop structure of gauge and gravity amplitudes and to unravel their UV properties.

\section{Acknowledgments}

We thank Nima Arkani-Hamed, Scott Davies, Tristan Dennen, Michael Green, Yu-tin Huang, Harald Ita, David Kosower, Kelly Stelle and Pierre Vanhove for many stimulating discussions. We thank Academic Technology Services at UCLA for computer support. This research was supported by the US Department of Energy under contracts DE-AC0276SF00515, DE-FG03-91ER40662 and DE-FG02-90ER40577 (OJI), and by the US National Science Foundation under grants PHY-0756174, PHY-0855356 and PHY05-51164. R. R. acknowledges support from the A. P. Sloan Foundation. H. J.'s research is supported by the European Research Council under Advanced Investigator Grant ERC-AdG-228301.

\section{Appendix A: Useful numerator functional equations}

In this appendix we list a set of numerator equations that determine the four-loop fourpoint $\mathcal{N}=4 \mathrm{sYM}$ amplitude (up to snail contributions), starting from the two planar master graphs 18 and 28 in fig. 4. These equations follow directly from the dual Jacobi relations. However, to make the equations more convenient for generating numerator factors from the our two planar master numerators, we performed various simplifications which follow from the $\mathcal{N}=4$ sYM auxiliary constraints described in section II B. In particular, we use the two-term relations (see eq. (A5)) which rely on the no one-loop triangle subgraph constraint to eliminate numerators appearing in other dual Jacobi relations. We also simplified the functional arguments of the numerators using the auxiliary constraint that numerators are independent of the loop momenta of one-loop box subgraphs. For example, instead of the dual relation,

$$
\begin{aligned}
N_{50}\left(k_{1}, k_{2}, k_{3}, l_{5}, l_{6}, l_{7}, l_{8}\right)= & N_{28}\left(k_{2}, k_{1}, k_{4}, l_{5}, k_{3}-l_{7}, k_{2}-l_{6}, l_{8}\right) \\
& -N_{28}\left(k_{1}, k_{2}, k_{3}, l_{6}, k_{4}-l_{8}, k_{1}-l_{5}, l_{7}\right),
\end{aligned}
$$

we simplify this to

$$
\begin{aligned}
N_{50}\left(k_{1}, k_{2}, k_{3}, l_{5}, l_{6}, l_{7}, l_{8}\right)= & N_{28}\left(k_{2}, k_{1}, k_{4}, l_{5}, k_{3}-l_{7}, l_{7}, l_{8}\right) \\
& -N_{28}\left(k_{1}, k_{2}, k_{3}, l_{6}, k_{4}-l_{8}, l_{7}, l_{8}\right),
\end{aligned}
$$


using the fact that $N_{28}$ is, in fact, independent of the values of the last two arguments since these momenta are those of one-loop box subgraphs (see fig. 4). In this sense, the last two arguments of $N_{28}$ are effectively placeholders, and can be assigned any value without altering the numerators. These simplifications, however, imply that the equations given below are specific to $\mathcal{N}=4$ sYM theory and will not hold for corresponding numerators of amplitudes of theories with fewer supersymmetries. They are also not in direct correspondence with the color-Jacobi equations, because numerators of graphs with triangle subgraphs are set to zero, although corresponding color factors are nonvanishing.

On the left-hand side of each duality equation, for simplicity, we will suppress the canonical arguments, which are the three external momenta and the four independent loop momenta following the graph labels in figs. 5-11, i.e.

$$
N_{i} \equiv N_{i}\left(k_{1}, k_{2}, k_{3}, l_{5}, l_{6}, l_{7}, l_{8}\right)
$$

and we take $k_{4} \equiv-k_{1}-k_{2}-k_{3}$ throughout. We have ordered the equations so that the substitutions that are required to express the given numerators in terms of the two master numerators always come from previous equations in the list. With the above notation, the 
required equations are

$$
\begin{aligned}
& N_{58}=N_{18}\left(k_{1}, k_{2}, k_{3}, k_{2}-l_{6}, l_{5}, l_{7}, l_{8}\right)-N_{18}\left(k_{2}, k_{1}, k_{3}, k_{1}-l_{6}, l_{5}, l_{7}, l_{8}\right), \\
& N_{33}=N_{28}\left(k_{4}, k_{3}, k_{2}, k_{3}-l_{5}, k_{2}-l_{6}+l_{7}, l_{7}, l_{8}\right)-N_{18}\left(k_{1}, k_{2}, k_{3}, k_{2}-l_{6}, k_{3}-l_{5}, l_{7}, l_{8}\right), \\
& N_{50}=N_{28}\left(k_{2}, k_{1}, k_{4}, l_{5}, k_{3}-l_{7}, l_{7}, l_{8}\right)-N_{28}\left(k_{1}, k_{2}, k_{3}, l_{6}, k_{4}-l_{8}, l_{7}, l_{8}\right) \text {, } \\
& N_{6}=-N_{33}\left(k_{1}, k_{2}, k_{4}, l_{7}, l_{5}-l_{6}, k_{1}-l_{6}, l_{8}\right)-N_{33}\left(k_{2}, k_{1}, k_{4}, l_{7}, l_{6}, k_{2}-l_{5}+l_{6}, l_{8}\right) \text {, } \\
& N_{14}=-N_{33}\left(k_{3}, k_{2}, k_{1}, l_{5},-l_{5}-l_{7}, k_{3}-l_{7}+l_{8}, l_{6}\right)-N_{33}\left(k_{3}, k_{2}, k_{1}, l_{5}, k_{2}+l_{7}, l_{7}-l_{8}, l_{8}\right) \text {, } \\
& N_{24}=-N_{28}\left(k_{1}, k_{2}, k_{3}, l_{5}-l_{7},-l_{6}, l_{7}, l_{8}\right)-N_{33}\left(k_{1}, k_{2}, k_{4},-l_{6},-l_{7},-l_{5}, l_{8}\right) \text {, } \\
& N_{32}=-N_{28}\left(k_{4}, k_{2}, k_{1}, l_{7}, k_{3}-l_{5}, l_{7}, l_{8}\right)-N_{33}\left(k_{2}, k_{1}, k_{3}, l_{5}, l_{6}, k_{2}+l_{5}+l_{6}-l_{7}, l_{8}\right) \text {, } \\
& N_{48}=N_{28}\left(k_{3}, k_{4}, k_{1}, l_{8}, k_{2}-l_{5}, l_{7}, l_{8}\right)-N_{33}\left(k_{1}, k_{2}, k_{3}, k_{3}-l_{6}, k_{2}-l_{5}, l_{7}, l_{8}\right) \text {, } \\
& N_{49}=-N_{33}\left(k_{1}, k_{2}, k_{3}, k_{3}-l_{8}, k_{2}-l_{5},-l_{7}, l_{8}\right)-N_{33}\left(k_{4}, k_{1}, k_{2}, l_{5},-l_{7}, l_{6}, l_{8}\right), \\
& N_{66}=N_{58}\left(k_{1}, k_{2}, k_{4}, l_{5}-k_{3}-l_{6}, l_{6}, l_{7}, l_{8}\right)-N_{58}\left(k_{1}, k_{2}, k_{3}, k_{3}+l_{6}, l_{6}, l_{7}, l_{8}\right) \text {, } \\
& N_{1}=-N_{6}\left(k_{1}, k_{2}, k_{3}, l_{6}, l_{5}, l_{7}, l_{8}\right)-N_{6}\left(k_{1}, k_{2}, k_{4}, l_{6}, l_{5}, l_{7}, l_{8}\right) \text {, } \\
& N_{68}=N_{14}\left(k_{1}, k_{2}, k_{3}, k_{1}-l_{5},-l_{6},-l_{7},-l_{8}\right)-N_{14}\left(k_{1}, k_{2}, k_{4}, l_{5}-k_{2},-l_{7},-l_{6}, l_{8}\right) \text {, } \\
& N_{21}=-N_{14}\left(k_{2}, k_{1}, k_{3}, l_{5}, l_{6}, l_{7}, l_{8}\right)-N_{18}\left(k_{2}, k_{1}, k_{3},-l_{5}, k_{1}+k_{3}+l_{5}-l_{6}, l_{7}, l_{8}\right) \text {, } \\
& N_{26}=N_{24}\left(k_{2}, k_{1}, k_{3},-l_{5},-k_{4}-l_{6}-l_{7}, l_{8}, l_{6}\right)-N_{24}\left(k_{2}, k_{1}, k_{4},-l_{5}, l_{7}-k_{3}, l_{6}-k_{1}-l_{5}-l_{8}, l_{6}\right) \text {, } \\
& N_{27}=-N_{18}\left(k_{2}, k_{1}, k_{4},-l_{5}, l_{7}, l_{7}, l_{8}\right)-N_{24}\left(k_{1}, k_{2}, k_{4}, l_{5},-k_{3}-l_{7}-l_{8}, k_{3}-l_{6}+l_{7}+l_{8}, l_{8}\right) \text {, } \\
& N_{37}=-N_{28}\left(k_{2}, k_{1}, k_{3}, k_{1}-l_{5}, k_{4}+l_{8}, l_{7}, l_{6}\right)-N_{49}\left(k_{2}, k_{1}, k_{3}, k_{1}-l_{5},-l_{8}, l_{7}-k_{2}, l_{6}\right) \text {, } \\
& N_{39}=N_{28}\left(k_{2}, k_{1}, k_{3},-l_{5}-l_{7}, k_{4}+l_{6}+l_{8}, l_{5}, l_{6}\right)-N_{48}\left(k_{1}, k_{2}, k_{3}, l_{7}, l_{8},-l_{5}-l_{7},-l_{6}-l_{8}\right) \text {, } \\
& N_{45}=N_{49}\left(k_{1}, k_{2}, k_{3}, l_{5}-l_{6}-l_{7}-l_{8}, k_{4}-l_{6}, l_{5}, l_{7}\right) \\
& +N_{49}\left(k_{1}, k_{2}, k_{4}, k_{2}+l_{6}+l_{7}+l_{8}, l_{7}, l_{5}, k_{4}-l_{6}\right), \\
& N_{38}=N_{49}\left(k_{2}, k_{1}, k_{4}, l_{6}, k_{3}+l_{5}+l_{7},-l_{5}+l_{6}, k_{4}-l_{8}\right) \\
& -N_{49}\left(k_{1}, k_{2}, k_{4}, l_{5}-l_{6}, k_{3}+l_{5}+l_{7},-l_{6}, l_{7}+l_{8}\right) \text {, } \\
& N_{53}=N_{58}\left(k_{1}, k_{2}, k_{3}, k_{3}-l_{8}, l_{6}, l_{7}, l_{8}\right)+N_{66}\left(k_{1}, k_{2}, k_{4}, l_{8},-k_{4}-l_{5}, l_{7}, l_{8}\right) \text {, } \\
& N_{12}=N_{18}\left(k_{4}, k_{3}, k_{2}, l_{6}, k_{2}+l_{8}, l_{5}, l_{7}\right)+N_{26}\left(k_{3}, k_{4}, k_{1},-l_{6}, l_{8},-l_{5}, l_{8}\right) \text {, } \\
& N_{51}=N_{18}\left(k_{3}, k_{2}, k_{1}, k_{1}+k_{2}-l_{5},-l_{6}, l_{7}, l_{8}\right)-N_{21}\left(k_{2}, k_{3}, k_{1}, l_{5}-k_{1}-k_{2},-l_{6}, l_{7}, l_{8}\right) \text {, } \\
& N_{63}=N_{21}\left(k_{1}, k_{2}, k_{3}, k_{2}-l_{5}, k_{1}+k_{2}-l_{5}-l_{6}, l_{7}, l_{8}\right) \\
& -N_{21}\left(k_{2}, k_{1}, k_{3}, k_{1}-l_{5}, k_{1}+k_{2}-l_{5}-l_{6}, l_{7}, l_{8}\right) \text {, } \\
& N_{79}=N_{45}\left(k_{1}, k_{2}, k_{3}, k_{2}-l_{5}, k_{4}-l_{7}, l_{6},-l_{6}-l_{8}\right) \\
& -N_{45}\left(k_{1}, k_{2}, k_{3}, l_{5}-k_{1}, l_{7}, k_{3}-l_{6}, k_{4}+l_{5}-l_{7}-l_{8}\right), \\
& N_{80}=N_{53}\left(k_{1}, k_{2}, k_{3}, k_{3}-l_{7}, l_{6}, l_{7}, l_{8}\right)+N_{53}\left(k_{1}, k_{2}, k_{3}, l_{7}-k_{4}, l_{5}, l_{6}, l_{8}\right) \text {, } \\
& N_{55}=N_{51}\left(k_{1}, k_{2}, k_{3}, k_{1}+l_{5}, l_{6}, l_{7}, l_{8}\right)-N_{51}\left(k_{1}, k_{3}, k_{2}, k_{1}+l_{5}, l_{6}, l_{7}, l_{8}\right) \text {, } \\
& N_{83}=-N_{55}\left(k_{3}, k_{1}, k_{2}, k_{1}+k_{2}-l_{5}, l_{8}, l_{6}, l_{7}\right)-N_{55}\left(k_{3}, k_{1}, k_{2}, l_{5}-k_{3}, l_{6}, l_{7}, l_{8}\right) \text {. }
\end{aligned}
$$

There are also a set of simpler two-term relations whenever one of the three numerators 
vanishes due to the appearance of a forbidden one-loop triangle subgraph,

$$
\begin{aligned}
& N_{5}=N_{4}=N_{3}=N_{2}=N_{1}, \\
& N_{11}=N_{10}=N_{9}=N_{8}=N_{7}=N_{6}, \\
& N_{40}=N_{13}=-N_{12}, \\
& N_{41}=-N_{17}=-N_{16}=-N_{15}=N_{14}, \\
& N_{42}=N_{20}=-N_{19}=N_{18}, \\
& N_{43}=-N_{23}=-N_{22}=-N_{21}, \\
& N_{25}=N_{24}, \\
& N_{44}=-N_{26}, \\
& N_{31}=-N_{30}=N_{29}=N_{28}, \\
& N_{46}=N_{34}=N_{32}, \\
& N_{36}=-N_{35}=-N_{33}, \\
& N_{47}=N_{38}, \\
& N_{72}=N_{52}=N_{51}, \\
& N_{74}=-N_{54}=-N_{53}, \\
& N_{73}=N_{57}=N_{56}=N_{55}, \\
& N_{76}=-N_{62}=-N_{61}=-N_{60}=-N_{59}=-N_{58}, \\
& N_{77}=-N_{65}=N_{64}=N_{63}, \\
& N_{78}=-N_{67}=N_{66}, \\
& N_{75}=N_{71}=N_{70}=N_{69}=N_{68}, \\
& N_{82}=N_{81}=N_{80}, \\
& N_{85}=N_{84}=N_{83} .
\end{aligned}
$$

Plain-text, computer-readable versions of both the original duality relations (using only the no one-loop triangle subgraph property) and the simplified ones presented above may be found online [38]. Many other functional equations can be obtained from the dual Jacobi

relations, which we will not list here. Although important, as they provide additional independent constraints to the full system, they are not needed to specify the solution once the system and master graph numerators have been solved. However, we have confirmed that the numerators presented in appendix B automatically satisfy all these remaining dual Jacobi relations.

\section{Appendix B: Explicit numerators for graphs}

In this appendix we give the explicit values $N_{i}$ of the distinct graph numerators in the $\mathcal{N}=4 \mathrm{sYM}$ amplitude. (The remaining ones are given directly in terms of these via eq. (A5).) These values are obtained by taking the numerators of the master graphs (3.14) and substituting their values into eq. (A4). We have performed some algebraic simplifications to obtain the results collected here. The $\mathcal{N}=8$ supergravity numerators are squares of the $N_{i}$, as in eqs. (3.2) and (4.16). 
The explicit values of the distinct numerators are

$$
\begin{aligned}
& N_{1}=s^{3} \text {, } \\
& N_{6}=\frac{1}{2} s^{2}\left(\tau_{45}-\tau_{35}-s\right) \text {, } \\
& N_{12}=\frac{1}{2} s\left(s\left(\tau_{16}-\tau_{26}-\tau_{35}+\tau_{45}+2 \tau_{56}+2 t\right)\right. \\
& \left.-2\left(4 \tau_{16} \tau_{25}+4 \tau_{15} \tau_{26}+\tau_{45}\left(\tau_{36}-3 \tau_{46}\right)+\tau_{35}\left(\tau_{46}-3 \tau_{36}\right)\right)\right), \\
& N_{14}=\frac{1}{4}\left(s\left(9 \tau_{15}^{2}+9 \tau_{25}^{2}+4 t^{2}+8 t \tau_{35}+2 \tau_{35}^{2}+2 \tau_{45}^{2}\right)+8 \tau_{25}\left(u^{2}-s^{2}\right)\right. \\
& \left.-5 t\left(\tau_{15} \tau_{35}+\tau_{25} \tau_{45}\right)+u\left(4 t\left(2 \tau_{15}+l_{5}^{2}\right)-5 \tau_{15} \tau_{45}-5 \tau_{25} \tau_{35}\right)\right), \\
& N_{18}=\frac{1}{4}\left(6 u^{2} \tau_{25}+u\left(2 s\left(5 \tau_{25}+2 \tau_{26}\right)-\tau_{15}\left(7 \tau_{16}+6 t\right)\right)\right. \\
& +t\left(\tau_{15} \tau_{26}-\tau_{25}\left(\tau_{16}+7 \tau_{26}\right)\right)+s\left(4 \tau_{15}\left(t-\tau_{26}\right)+6 \tau_{36}\left(\tau_{35}-\tau_{45}\right)\right. \\
& \left.\left.-\tau_{16}\left(4 t+5 \tau_{25}\right)-\tau_{46}\left(5 \tau_{35}+\tau_{45}\right)\right)+2 s^{2}\left(t+\tau_{26}-\tau_{35}+\tau_{36}+\tau_{56}\right)\right), \\
& N_{21}=\frac{1}{4}\left(t\left(12 \tau_{15}^{2}-7 \tau_{15} \tau_{16}+\tau_{25}\left(\tau_{16}-10 \tau_{35}\right)\right)+u\left(\tau_{25}\left(12 \tau_{25}-8 t-7 \tau_{26}\right)+\tau_{15}\left(8 t+\tau_{26}-10 \tau_{35}\right)\right)\right. \\
& +4 l_{5}^{2}\left(u^{2}-s t\right)-s\left(2 \tau_{15}\left(6 \tau_{16}+5 \tau_{25}\right)+4 u\left(\tau_{16}-t+\tau_{26}+2 \tau_{45}\right)+\tau_{35}\left(\tau_{36}-12 \tau_{35}-10 \tau_{46}\right)\right. \\
& \left.\left.-\tau_{45}\left(11 \tau_{36}+6 \tau_{46}\right)+12 \tau_{25} \tau_{26}\right)+2 s^{2}\left(u-\tau_{16}-\tau_{35}+\tau_{36}-\tau_{56}\right)\right), \\
& N_{24}=\frac{1}{4}\left(s\left(2 \tau_{36}\left(3 \tau_{35}+\tau_{45}\right)+\tau_{46}\left(\tau_{35}+13 \tau_{45}\right)-4 u\left(2 \tau_{15}+\tau_{16}-2 \tau_{25}+\tau_{26}\right)-11 \tau_{15} \tau_{26}\right)\right. \\
& -2 s^{2}\left(2 \tau_{15}-2 \tau_{25}+\tau_{26}-\tau_{36}-\tau_{37}+\tau_{47}-\tau_{56}\right)+u\left(11 \tau_{16} \tau_{25}+\tau_{15}\left(7 \tau_{16}+\tau_{26}\right)\right) \\
& \left.+t \tau_{25}\left(12 \tau_{16}+7 \tau_{26}\right)\right) \text {, } \\
& N_{26}=\frac{1}{4}\left(u \tau_{15}\left(7 \tau_{16}+12 \tau_{26}\right)+t\left(11 \tau_{15} \tau_{26}+\tau_{25}\left(\tau_{16}+7 \tau_{26}\right)\right)+s\left(16 \tau_{15} \tau_{17}\right.\right. \\
& -4 u\left(2 \tau_{15}+\tau_{16}-2 \tau_{25}+\tau_{26}\right)+\tau_{25}\left(16 \tau_{27}-11 \tau_{16}\right)+\tau_{35}\left(6 \tau_{36}-4 \tau_{37}+\tau_{46}-20 \tau_{47}\right) \\
& \left.\left.+\tau_{45}\left(2 \tau_{36}-20 \tau_{37}+13 \tau_{46}-4 \tau_{47}\right)\right)+2 s^{2}\left(\tau_{17}-2 \tau_{15}+2 \tau_{25}-\tau_{26}-\tau_{27}+\tau_{36}+\tau_{56}+2 \tau_{57}\right)\right) \text {, } \\
& N_{27}=\frac{1}{4}\left(u \tau_{18}\left(\tau_{25}-7 \tau_{15}\right)+\tau_{28}\left(t\left(\tau_{15}-11 \tau_{25}\right)-4 u \tau_{25}\right)+s\left(4 \tau_{15}\left(3 \tau_{17}+\tau_{18}-\tau_{27}\right)\right.\right. \\
& +4 u\left(2 \tau_{15}-2 \tau_{25}+\tau_{28}\right)+\tau_{45}\left(5 \tau_{18}+5 \tau_{28}-16 \tau_{37}-5 \tau_{38}\right)+\tau_{35}\left(2 \tau_{38}-16 \tau_{47}-9 \tau_{48}\right)-4 \tau_{18} t \\
& \left.\left.-4 \tau_{25}\left(\tau_{17}-3 \tau_{27}\right)\right)+2 s^{2}\left(2 \tau_{15}+2 \tau_{17}-2 \tau_{25}+\tau_{28}-\tau_{36}+2 \tau_{37}+\tau_{38}+\tau_{46}+2 \tau_{57}+\tau_{58}\right)\right) \text {, } \\
& N_{28}=\frac{1}{4}\left(s\left(2 \tau_{15} t+\tau_{16}\left(2 t-5 \tau_{25}+\tau_{35}\right)+5 \tau_{35}\left(\tau_{26}+\tau_{36}\right)+2 t\left(2 \tau_{46}-\tau_{56}\right)-10 u \tau_{25}\right)\right. \\
& \left.-4 s^{2} \tau_{25}-6 u\left(\tau_{46}\left(t-\tau_{25}+\tau_{45}\right)+\tau_{25} \tau_{26}\right)-t\left(\tau_{15}\left(4 \tau_{36}+5 \tau_{46}\right)+5 \tau_{25} \tau_{36}\right)\right), \\
& N_{32}=\frac{1}{4}\left(t\left(\tau_{25}\left(\tau_{16}-11 \tau_{25}-12 \tau_{26}\right)+\tau_{25} \tau_{35}-6 \tau_{35}^{2}-\tau_{15}\left(\tau_{26}-4 \tau_{45}\right)\right)-u\left(5 \tau_{25} \tau_{26}\right.\right. \\
& \left.+\tau_{15}\left(7 \tau_{16}-5 \tau_{25}+5 \tau_{35}\right)\right)+s\left(\tau_{15}\left(5 \tau_{16}-4 t\right)+\tau_{16}\left(8 t+\tau_{25}\right)+\tau_{35} \tau_{36}+5 \tau_{45}\left(\tau_{25}-\tau_{35}-2 \tau_{36}\right)\right. \\
& \left.\left.-\tau_{46}\left(11 \tau_{35}+6 \tau_{45}\right)+2 u\left(6 \tau_{25}+4 \tau_{26}-4 \tau_{35}-2 l_{5}^{2}\right)\right)+2 s^{2}\left(3 \tau_{25}-\tau_{35}-\tau_{36}-3 \tau_{46}+\tau_{56}\right)\right) \text {, } \\
& N_{33}=\frac{1}{4}\left(s^{2}\left(4 \tau_{17}-2\left(4 \tau_{26}+\tau_{35}+2 \tau_{36}\right)\right)-6 u^{2} \tau_{35}+u\left(\left(4 \tau_{16}+5 \tau_{26}\right) \tau_{45}\right.\right. \\
& \left.-\tau_{17}\left(11 \tau_{25}+7 \tau_{35}+6 \tau_{45}\right)+\tau_{35}\left(11 \tau_{37}-5 \tau_{46}\right)\right)-t\left(5 \tau_{17} \tau_{25}+6 \tau_{15} \tau_{26}+\left(6 \tau_{26}-5 \tau_{17}-4 \tau_{27}\right) \tau_{45}\right. \\
& \left.+\tau_{35}\left(7 \tau_{26}-11 \tau_{36}+5 \tau_{47}\right)\right)+s\left(\tau_{15}\left(5 \tau_{26}+4 \tau_{46}\right)-5 \tau_{35}\left(\tau_{16}+\tau_{27}\right)+4 \tau_{25} \tau_{47}+2 u\left(5 \tau_{17}+2 \tau_{25}\right.\right. \\
& \left.\left.\left.-5\left(\tau_{26}+\tau_{35}\right)+\tau_{56}\right)+2 t\left(\tau_{16}-\tau_{15}-\tau_{27}+\tau_{57}\right)\right)\right) \text {, } \\
& N_{37}=\frac{1}{4}\left(u^{2}\left(4 \tau_{15}-2 \tau_{27}\right)-2 s^{2}\left(2 \tau_{15}+3 \tau_{27}+4 \tau_{36}\right)+t\left(6 \tau_{26} \tau_{27}+5 \tau_{27} \tau_{35}-6 \tau_{27} \tau_{36}\right.\right. \\
& \left.+6 \tau_{36} \tau_{37}-\tau_{25}\left(5 \tau_{36}+4 \tau_{46}\right)-\tau_{15}\left(6 \tau_{26}+5 \tau_{46}\right)+4 \tau_{35} \tau_{47}\right)+u\left(5 \tau_{27} \tau_{46}-6 \tau_{35} \tau_{36}+\left(5 \tau_{27}\right.\right. \\
& \left.\left.+4 \tau_{37}\right) \tau_{45}+\tau_{17}\left(5 \tau_{36}+4 \tau_{46}-2 t\right)+\tau_{15}\left(12 \tau_{36}+5 \tau_{37}+6 \tau_{46}-5 \tau_{47}\right)+2 t\left(\tau_{57}-\tau_{25}\right)\right) \\
& +s\left(6 \tau_{25} \tau_{27}-\left(4 \tau_{26}+5 \tau_{36}\right) \tau_{45}-5\left(\tau_{26}+\tau_{46}\right) \tau_{47}-\tau_{16}\left(4 t-5 \tau_{27}+\tau_{47}\right)-\tau_{15}\left(11 \tau_{26}+12 \tau_{27}\right.\right. \\
& \left.\left.\left.+6 \tau_{37}+11 \tau_{47}\right)+2 t\left(\tau_{35}-\tau_{56}\right)+2 u\left(t+2 \tau_{26}-5 \tau_{27}-8 \tau_{36}+2 \tau_{37}+\tau_{67}\right)\right)\right) \text {, }
\end{aligned}
$$




$$
\begin{aligned}
& N_{38}=\frac{1}{4}\left(t \left(\tau_{16} \tau_{25}+6 \tau_{25}^{2}-11 \tau_{25} \tau_{26}-\tau_{25} \tau_{35}+11 \tau_{35}^{2}+7 \tau_{35} \tau_{37}+\tau_{37} \tau_{45}+\tau_{15}\left(\tau_{45}-4 \tau_{16}\right)\right.\right. \\
& \left.-\tau_{35} \tau_{47}\right)-s\left(5 \tau_{15}^{2}-6 \tau_{17} \tau_{25}+20 \tau_{17} \tau_{26}+6 \tau_{25} \tau_{27}+4 \tau_{26} \tau_{27}+4 \tau_{16}\left(\tau_{17}+5 \tau_{27}\right)-9 \tau_{25} \tau_{36}\right. \\
& -11 \tau_{35} \tau_{36}-16 \tau_{36} \tau_{37}-5 \tau_{37} \tau_{45}+10 \tau_{35} \tau_{46}+5 \tau_{45} \tau_{46}-4\left(\tau_{35}+4 \tau_{46}\right) \tau_{47}+\tau_{15}\left(\tau_{37}+\tau_{47}\right. \\
& \left.\left.-4 \tau_{27}+10 \tau_{35}-9 \tau_{36}\right)\right)+u\left(\tau_{45}\left(7 \tau_{47}-5\left(2 \tau_{35}+\tau_{45}\right)\right)-4 \tau_{25} \tau_{26}+\tau_{15}\left(\tau_{26}-11 \tau_{16}\right)\right) \\
& +2 s\left(4 t \tau_{37}+u\left(4\left(\tau_{16}+\tau_{25}-\tau_{26}-\tau_{35}+\tau_{47}\right)+2 l_{5}^{2}\right)+s\left(2 \tau_{16}-2 \tau_{17}+2 \tau_{25}-2 \tau_{26}\right.\right. \\
& \left.\left.\left.-2 \tau_{27}-2 \tau_{35}+\tau_{56}-\tau_{57}+2 \tau_{67}\right)\right)\right) \text {, } \\
& N_{39}=\frac{1}{4}\left(t\left(\tau_{15}\left(12 \tau_{16}+6 \tau_{26}+\tau_{36}\right)-10 \tau_{38} \tau_{45}+\tau_{27}\left(5 \tau_{26}+12\left(\tau_{36}+\tau_{46}\right)\right)+\tau_{35}\left(\tau_{48}-7 \tau_{38}\right)\right)\right. \\
& -u\left(\tau_{17}\left(7 \tau_{16}+\tau_{26}\right)+11 \tau_{16} \tau_{27}-\tau_{25}\left(4 \tau_{36}+5 \tau_{46}\right)+\tau_{45}\left(9 \tau_{38}+6 \tau_{46}+7 \tau_{48}\right)\right)+s\left(4 \tau_{17} \tau_{18}\right. \\
& +11 \tau_{17} \tau_{26}+20 \tau_{18} \tau_{27}+20 \tau_{17} \tau_{28}+4 \tau_{27} \tau_{28}+\tau_{15}\left(5 \tau_{16}-11 \tau_{18}+10 \tau_{26}+5 \tau_{28}\right)-4 \tau_{18} \tau_{35} \\
& +4 \tau_{26} \tau_{35}-6 \tau_{35} \tau_{38}-16 \tau_{37} \tau_{38}+2 t\left(\tau_{25}+\tau_{36}+4 \tau_{38}\right)+4 \tau_{28} \tau_{45}-5 \tau_{25} \tau_{46}-\tau_{37} \tau_{46} \\
& -11 \tau_{45} \tau_{46}-13 \tau_{46} \tau_{47}-2 \tau_{36}\left(3 \tau_{37}+\tau_{47}\right)+2\left(3 \tau_{35}-\tau_{45}-8 \tau_{47}\right) \tau_{48} \\
& \left.+2 u\left(3 \tau_{15}+4 \tau_{17}-4 \tau_{27}+3 \tau_{46}+4 \tau_{48}+\tau_{56}\right)\right) \\
& \left.-2 s^{2}\left(u-\tau_{15}-2 \tau_{17}+2 \tau_{18}+\tau_{26}+2 \tau_{27}+2 \tau_{28}+\tau_{35}-\tau_{46}+\tau_{58}+\tau_{67}+2 \tau_{78}\right)\right), \\
& N_{45}=\frac{1}{4}\left(8 u^{2} \tau_{15}+t\left(\tau_{15}\left(\tau_{16}+7 \tau_{17}+\tau_{26}+\tau_{27}-5 \tau_{35}+5 \tau_{45}\right)-\left(\tau_{16}+\tau_{17}+8 t\right) \tau_{25}\right)\right. \\
& +s\left(\tau_{15}\left(12 \tau_{17}-18 \tau_{25}+11 \tau_{26}+\tau_{27}\right)+2\left(5 \tau_{16} \tau_{25}-2 \tau_{17} t-2 t\left(6 \tau_{25}+\tau_{26}\right)+3 \tau_{25}\left(\tau_{26}+2 \tau_{27}\right)\right)\right. \\
& \left.+11 \tau_{35}^{2}+\tau_{45}\left(6\left(\tau_{45}-2 \tau_{46}-\tau_{47}\right)-\tau_{36}-11 \tau_{37}\right)+\tau_{35}\left(\tau_{37}-5 \tau_{36}+13 \tau_{45}-10 \tau_{47}\right)\right) \\
& +u\left(4 s\left(\tau_{16}-t+\tau_{27}+2 \tau_{35}\right)-6 \tau_{15} \tau_{16}+\tau_{25}\left(7 \tau_{26}+7 \tau_{27}-5 \tau_{35}+5 \tau_{45}\right)+4 t l_{5}^{2}\right) \\
& \left.+2 s^{2}\left(\tau_{16}+\tau_{27}+5 \tau_{35}+\tau_{36}+\tau_{37}+\tau_{38}+3 \tau_{45}-\tau_{48}-\tau_{56}+\tau_{57}\right)\right) \text {, } \\
& N_{48}=\frac{1}{4}\left(s\left(\tau_{18}\left(5 \tau_{25}+4 \tau_{35}\right)+\tau_{36}\left(\tau_{15}-5 \tau_{27}-4 \tau_{45}\right)-\left(\tau_{17}-5 \tau_{25}\right) \tau_{46}+5 \tau_{35} \tau_{48}\right)-2 s u t\right. \\
& -5 \tau_{15} \tau_{18} t-t\left(5 \tau_{17} \tau_{26}+7 \tau_{35} \tau_{36}+\tau_{25}\left(11 \tau_{26}-5 \tau_{38}\right)+\tau_{15}\left(5 \tau_{28}+\tau_{38}\right)+4 \tau_{37} \tau_{46}\right. \\
& \left.+\tau_{36} \tau_{47}-4 \tau_{16}\left(\tau_{45}+\tau_{47}\right)\right)+u\left(4 \tau_{15} \tau_{26}+6 \tau_{25} \tau_{28}+\tau_{16}\left(7 \tau_{17}+\tau_{25}-4 \tau_{35}\right)+\tau_{36}\left(11 \tau_{37}\right.\right. \\
& \left.\left.+\tau_{45}\right)-4 \tau_{26}\left(\tau_{17}+\tau_{47}\right)+6\left(\tau_{45}-\tau_{25}\right) \tau_{48}\right)+2\left(s^{2}\left(\tau_{25}-\tau_{35}+2 \tau_{36}\right)+3 u t\left(\tau_{17}+\tau_{48}\right)\right. \\
& \left.\left.+s\left(u\left(3 \tau_{25}+\tau_{26}+4 \tau_{36}-\tau_{56}\right)+t\left(\tau_{15}-\tau_{16}-2 \tau_{17}-\tau_{18}-\tau_{47}-2 \tau_{48}+\tau_{58}+\tau_{67}\right)\right)\right)\right), \\
& N_{49}=\frac{1}{4}\left(2 s^{2} t-t\left(11 \tau_{35} \tau_{38}+2 \tau_{15}\left(2 \tau_{36}+5 \tau_{46}\right)+\tau_{25}\left(11 \tau_{28}-4 \tau_{37}+4 \tau_{38}+\tau_{47}\right)\right.\right. \\
& \left.+4 \tau_{27} \tau_{48}+5 \tau_{17}\left(\tau_{35}+\tau_{48}\right)\right)+s\left(\tau_{15}\left(11 \tau_{17}+5 \tau_{38}\right)-5 \tau_{17} \tau_{28}-2 \tau_{16}\left(5 \tau_{25}+2 \tau_{35}\right)+\tau_{45}\left(5 \tau_{46}\right.\right. \\
& \left.\left.-4\left(\tau_{18}+\tau_{37}\right)\right)+5 \tau_{38}\left(\tau_{27}-\tau_{47}\right)-4 \tau_{28} \tau_{47}+\tau_{25}\left(7 \tau_{27}+5 \tau_{48}-5 \tau_{26}\right)\right)+u\left(\tau_{27}\left(11 \tau_{38}+4 \tau_{45}\right)\right. \\
& -\tau_{25}\left(5 \tau_{16}-5 \tau_{18}+11 \tau_{26}+\tau_{37}\right)-\tau_{17}\left(6 \tau_{18}-12 \tau_{38}+\tau_{45}\right)+\left(7 \tau_{25}+6 \tau_{35}\right) \tau_{46}+\tau_{38}\left(5 \tau_{45}+6 \tau_{47}\right) \\
& \left.+\tau_{15}\left(\tau_{46}-4\left(\tau_{47}+\tau_{48}\right)\right)\right)+2\left(s^{2}\left(\tau_{25}-4 \tau_{17}-\tau_{35}+2 \tau_{38}\right)+u\left(4 u \tau_{17}+t\left(2 \tau_{27}-2 \tau_{15}\right.\right.\right. \\
& \left.\left.\left.\left.-2 \tau_{25}-3 \tau_{46}+\tau_{57}\right)\right)+s\left(\tau_{16} t+u\left(\tau_{28}+4 \tau_{38}-\tau_{58}\right)-t\left(\tau_{18}+2 \tau_{37}-2 \tau_{46}+\tau_{56}+\tau_{78}\right)\right)\right)\right), \\
& N_{50}=\frac{1}{4}\left(t\left(\tau_{25}\left(5 \tau_{37}+4 \tau_{47}\right)-\tau_{38}\left(4 \tau_{16}+5 \tau_{26}\right)-5 \tau_{15} \tau_{27}\right)+s\left(\tau_{45}\left(4 \tau_{27}+5 \tau_{37}\right)\right.\right. \\
& \left.-\tau_{18}\left(5 \tau_{26}+4 \tau_{36}\right)-5 \tau_{15} \tau_{47}+5\left(\tau_{16}-\tau_{36}\right) \tau_{48}\right)+u\left(6 \tau_{35} \tau_{37}-6 \tau_{26} \tau_{28}+\tau_{15}\left(11 \tau_{17}-\tau_{37}\right)\right. \\
& \left.+\left(11 \tau_{16}+6\left(2 \tau_{26}+\tau_{36}\right)\right) \tau_{48}\right)+2 t\left(3 u\left(\tau_{15}-\tau_{26}+\tau_{37}-\tau_{48}\right)+s\left(\tau_{18}-2 \tau_{15}+2 \tau_{26}-\tau_{27}\right.\right. \\
& \left.\left.\left.+\tau_{36}-2 \tau_{37}-\tau_{45}+2 \tau_{48}+\tau_{57}-\tau_{68}\right)\right)\right) \text {, } \\
& N_{51}=\frac{1}{2}\left(4 t^{2}\left(\tau_{15}+\tau_{25}\right)-6 \tau_{15}^{2} t-4 s t(s-u)-6 u \tau_{25}^{2}+\tau_{35}\left(5 t \tau_{25}-6 s \tau_{35}\right)\right. \\
& \left.+5 \tau_{15}\left(s \tau_{25}+u \tau_{35}\right)+14 s^{2} \tau_{45}-s\left(6 u \tau_{15}+t\left(\tau_{15}+6 \tau_{25}+13 \tau_{35}-2 l_{5}^{2}\right)\right)-2 u^{2} l_{5}^{2}\right) \text {, } \\
& N_{53}=8 s\left(t \tau_{35}+u \tau_{45}-s \tau_{25}\right) \text {, } \\
& N_{55}=\frac{1}{2} t\left(t\left(\tau_{25}-8 \tau_{15}+5 \tau_{45}\right)+u\left(9 \tau_{45}-17 \tau_{15}\right)\right) \text {, } \\
& N_{58}=s\left(2 u\left(\tau_{45}-3 \tau_{35}\right)-s\left(u-t+4 \tau_{25}+5 \tau_{35}+\tau_{45}\right)\right) \text {, }
\end{aligned}
$$




$$
\begin{aligned}
N_{63} & =-\frac{1}{2} s\left(5 t \tau_{35}+u\left(12 \tau_{36}+5 \tau_{45}-4 \tau_{46}\right)+s\left(2 t-2 u+2 \tau_{25}+2 \tau_{46}+8 \tau_{26}+10 \tau_{36}-7 \tau_{15}\right)\right), \\
N_{66} & =s\left(4 t\left(\tau_{35}-2 \tau_{36}\right)+2 u\left(\tau_{35}+3 \tau_{45}-4 \tau_{46}\right)-s\left(6 u+\tau_{15}-6 t+5 \tau_{25}-8 \tau_{26}\right)\right), \\
N_{68} & =\frac{1}{2} s\left(s\left(2\left(\tau_{15}+t-u\right)-7 \tau_{25}\right)+5 u \tau_{35}+5 t \tau_{45}\right), \\
N_{79} & =\frac{1}{2} s\left(5 t \tau_{35}+u\left(5 \tau_{45}+4 \tau_{46}-4 \tau_{37}+12 \tau_{47}-12 \tau_{36}\right)\right. \\
& \left.+s\left(2\left(\tau_{16}+\tau_{25}-\tau_{27}+3 \tau_{17}-3 \tau_{26}+4 \tau_{47}-4 \tau_{36}\right)-7 \tau_{15}\right)\right), \\
N_{80} & =16 s^{2}(u-t), \\
N_{83} & =-\frac{9}{2} k_{4}^{2} s(u-t) .
\end{aligned}
$$

The other numerators are given directly in terms of these in eq. (A5). For $N_{83}$ it should be understood that $k_{4}^{2} \rightarrow 0$ only after canceling the $1 / k_{4}^{2}$ propagator. Alternatively, we can

rewrite the snail contributions in terms of the numerators of the graphs in fig. 12, we have

$$
N_{83^{\prime}}=N_{84^{\prime}}=N_{85^{\prime}}=-\frac{9}{2} s(u-t) .
$$

Plain-text, computer-readable versions of these expressions may be found online [38].

It is interesting to note that $N_{33}$ can be used as a non-planar master graph numerator, as discussed in section IIIB. This implies that the single numerator $N_{33}$ contains the same amplitude-specific information as the two planar master numerators $N_{18}$ and $N_{28}$ combined.

\section{Appendix C: Vacuum integrals and their expression in terms of master integrals}

In the first column of table I we give the numerators of the vacuum integrals in figs. 18-20, as they appear in the expression for $\widetilde{\mathcal{V}}^{(4)}$ defined in eqs. (4.20) and (4.21). Each integral can be reduced to a linear combination of the three master integrals $V_{1}, V_{2}$ and $V_{8}$. The second, third and fourth columns of the table provide the coefficients of $V_{1}, V_{2}$ and $V_{8}$, respectively, after this reduction. To obtain the coefficient of each vacuum integral $V_{i}$ in the final formula for the four-loop UV divergence in eq. (4.37), we simply sum the numbers in each column labeled by a $V_{i}$, to obtain

$$
\widetilde{\mathcal{V}}^{(4)}=\frac{23}{2}\left(V_{1}+2 V_{2}+V_{8}\right) .
$$

Inserting this value into eq. (4.20) yields the final result (4.37). 
TABLE I: Effective numerators for the vacuum integrals $I_{i}^{v}$ entering the UV pole of the fourloop $\mathcal{N}=8$ supergravity amplitude, and the coefficients arising from writing them as a linear combination of basis vacuum integrals $V_{1}, V_{2}$, and $V_{8}$.

\begin{tabular}{|c|c|c|c|c|}
\hline$I^{v}$ & Effective numerator & $V_{1}$ & $V_{2}$ & $V_{8}$ \\
\hline$I_{1}^{v}$ & $-\frac{117674}{1485}$ & 0 & $-\frac{117674}{1485}$ & 0 \\
\hline$I_{2}^{v}$ & $\frac{19112}{1485} \tau_{a, b}^{2}$ & $\frac{8798687}{5346000}$ & $\frac{212621}{27000}$ & 0 \\
\hline$I_{3}^{v}$ & $\frac{9556}{145} \tau_{a, b}^{2}$ & $\frac{15937019}{178000}$ & $-\frac{140951}{33000}$ & 0 \\
\hline$I_{4}^{v}$ & $-\frac{16427}{495}$ & $-\frac{16427}{195}$ & 0 & 0 \\
\hline$I_{5}^{v}$ & $\frac{19112}{1485} \tau_{a, c}-\frac{19112}{1485} \tau_{b, c}$ & $-\frac{2389}{2070}$ & $-\frac{2389}{1485}$ & 0 \\
\hline$I_{6}^{v}$ & $-\frac{4778}{495} \tau_{a, c}+\frac{4778}{1485} \tau_{b, c}$ & $\frac{16723}{2970}$ & $-\frac{4778}{1485}$ & 0 \\
\hline$I_{7}^{v}$ & $-\frac{9556}{1485}$ & $\frac{109894}{7425}$ & $\frac{90782}{2475}$ & 0 \\
\hline$I_{8}^{v}$ & $\frac{38224}{1485} \tau_{a, b}$ & $-\frac{2389}{675}$ & $-\frac{19112}{2475}$ & 0 \\
\hline$I_{9}^{v}$ & $\frac{38224}{1485} \tau_{a, b}^{2}$ & $-\frac{1617353}{148500}$ & $\frac{2606399}{74250}$ & 0 \\
\hline$I_{10}^{v}$ & $-\frac{19112}{1485} \tau_{a, c}-\frac{19112}{1485} \tau_{b, c}$ & $-\frac{2389}{2970}$ & $-\frac{2389}{1485}$ & 0 \\
\hline$I_{11}^{v}$ & $-\frac{38224}{1485} \tau_{a, b}$ & $\frac{90782}{22275}$ & $-\frac{9556}{825}$ & 0 \\
\hline$I_{12}^{v}$ & $-\frac{19112}{1485}$ & $\frac{31057}{990}$ & $\frac{38224}{495}$ & 0 \\
\hline$I_{13}^{v}$ & $\frac{10048}{99}$ & $\frac{2512}{99}$ & $\frac{10048}{99}$ & 0 \\
\hline$I_{14}^{v}$ & $-\frac{19112}{1485}$ & $-\frac{4778}{275}$ & $-\frac{324904}{7425}$ & 0 \\
\hline$I_{15}^{v}$ & $\frac{19112}{1485}$ & $\frac{66892}{4455}$ & $\frac{19112}{495}$ & 0 \\
\hline$I_{16}^{v}$ & $\frac{19112}{1485} \tau_{a, b}^{2}$ & $\frac{977101}{267200}$ & $\frac{88393}{11950}$ & 0 \\
\hline$I_{17}^{v}$ & $\frac{39676}{145}$ & $\begin{array}{l}267300 \\
9919\end{array}$ & $\begin{array}{l}14850 \\
19838 \\
\end{array}$ & 0 \\
\hline & $\begin{array}{l}1485 \\
9556 \\
\tau^{2}\end{array}$ & $\begin{array}{l}495 \\
147791\end{array}$ & $\begin{array}{c}\overline{1485} \\
661753\end{array}$ & 2389 \\
\hline${ }^{1} 18$ & $\frac{1000}{1485} \tau_{a, b}^{2}$ & $\frac{14101}{297000}$ & $\frac{00150}{148500}$ & $\frac{\frac{2039}{396}}{x}$ \\
\hline$I_{19}^{v}$ & $-\frac{64441}{1485}$ & 0 & 0 & $-\frac{64441}{1485}$ \\
\hline$I_{20}^{v}$ & $\frac{38224}{1485} \tau_{a, b}$ & $-\frac{102727}{14850}$ & $-\frac{74059}{7425}$ & 0 \\
\hline$I_{21}^{v}$ & $\frac{5284}{1485}$ & $\frac{18494}{7}$ & $\frac{34346}{732}$ & 0 \\
\hline$I_{\nu}^{v}$ & $\begin{array}{l}1485 \\
934 \\
\end{array}$ & $\begin{array}{l}7425 \\
467\end{array}$ & $\begin{array}{l}7425 \\
1868\end{array}$ & 934 \\
\hline$I_{22}$ & $\overline{165}$ & $\frac{165}{165}$ & 165 & $-\frac{\overline{165}}{165}$ \\
\hline$I_{23}^{v}$ & $\frac{526}{135} \tau_{a, b}-\frac{91}{1485} \tau_{a, c}$ & $\frac{279199}{297000}$ & $\frac{72052}{37125}$ & 0 \\
\hline$I_{24}^{v}$ & $\frac{3736}{495} \tau_{a, b}$ & $\frac{26152}{12375}$ & $-\frac{91532}{12375}$ & 0 \\
\hline$I_{25}^{v}$ & $-\frac{9556}{1485} \tau_{a, b}$ & $-\frac{2389}{2475}$ & $-\frac{16723}{7425}$ & 0 \\
\hline$I_{26}^{v}$ & $-\frac{11048}{1485}$ & $-\frac{17953}{2475}$ & $-\frac{44192}{2475}$ & 0 \\
\hline$I_{27}^{v}$ & $-\frac{1228}{125}$ & $-\frac{307}{50}$ & $-\frac{10488}{675}$ & 0 \\
\hline$I_{28}^{v}$ & $-\frac{3736}{495}$ & $\frac{934}{825}$ & $-\frac{1494}{825}$ & 0 \\
\hline$I_{29}^{v}$ & $\frac{3736}{495} \tau_{a, b}$ & $-\frac{48568}{4125}$ & $\frac{76588}{4125}$ & 0 \\
\hline$I_{30}^{v}$ & $\frac{934}{495}$ & $\frac{90131}{24750}$ & $\frac{119552}{12375}$ & 0 \\
\hline$I_{31}^{v}$ & $\frac{4778}{1485} \tau_{a, b}+\frac{9556}{1485} \tau_{a, c}$ & -45391 & $-\frac{112283}{1228}$ & 0 \\
\hline & $19112 \tau^{2}$ & 2721071 & $\begin{array}{r}14850 \\
-327293 \\
\end{array}$ & -2389 \\
\hline${ }^{1} 32$ & 1485 a,b & $\overline{148500}$ & $-\frac{74250}{70}$ & $-\frac{495}{495}$ \\
\hline$I_{33}^{v}$ & $-\frac{3736}{495} \tau_{a, b}$ & $-\frac{1868}{2475}$ & $-\frac{1868}{275}$ & $\frac{1868}{165}$ \\
\hline$I_{34}^{v}$ & $\frac{4778}{297}$ & $-\frac{155285}{2376}$ & $-\frac{47780}{297}$ & 0 \\
\hline$I_{35}^{v}$ & $-\frac{7904}{1485}$ & $-\frac{3952}{495}$ & $-\frac{27664}{1485}$ & 0 \\
\hline
\end{tabular}




\begin{tabular}{|c|c|c|c|c|}
\hline$I^{v}$ & Effective numerator & $V_{1}$ & $V_{2}$ & $V_{8}$ \\
\hline$I_{36}^{v}$ & $-\frac{3736}{495} \tau_{a, b}$ & $\frac{467}{4950}$ & $\frac{7939}{2475}$ & 0 \\
\hline$I_{37}^{v}$ & $\frac{9556}{1485} \tau_{a, b}$ & $-\frac{45391}{59400}$ & $-\frac{54947}{29700}$ & 0 \\
\hline$I_{38}^{v}$ & $\frac{3736}{495}$ & $\frac{21482}{2475}$ & $\frac{76588}{2475}$ & 0 \\
\hline$I_{39}^{v}$ & $\frac{4778}{495}$ & $\frac{45391}{6600}$ & $\frac{54947}{3300}$ & 0 \\
\hline$I_{40}^{v}$ & $-\frac{1228}{135}$ & $-\frac{3991}{225}$ & $-\frac{9824}{225}$ & 0 \\
\hline$I_{41}^{v}$ & $\frac{1868}{195} \tau_{a, b}$ & $\frac{47167}{9900}$ & $-\frac{20081}{4050}$ & 0 \\
\hline$I_{42}^{v}$ & $\frac{1228}{135} \tau_{a, b}+\frac{6524}{297} \tau_{a, c}$ & $\frac{257243}{14850}$ & $-\frac{16723}{2475}$ & 0 \\
\hline$I_{43}^{v}$ & $\frac{9556}{1485} \tau_{a, b}$ & $-\frac{16723}{14850}$ & $-\frac{2389}{825}$ & 0 \\
\hline$I_{44}^{v}$ & $\frac{19112}{1485} \tau_{a, b}^{2}$ & $-\frac{2047373}{133650}$ & $\frac{212621}{7425}$ & 0 \\
\hline$I_{45}^{v}$ & $\frac{10204}{1485}$ & $\frac{323977}{29700}$ & $\frac{135203}{4950}$ & 0 \\
\hline$I_{46}^{v}$ & $\frac{4778}{495}$ & $\frac{16723}{3960}$ & $\frac{2389}{220}$ & 0 \\
\hline$I_{47}^{v}$ & $-\frac{9556}{1485}$ & $-\frac{31057}{2970}$ & $-\frac{38224}{1485}$ & 0 \\
\hline$I_{48}^{v}$ & $-\frac{4778}{495}$ & $-\frac{4778}{825}$ & $-\frac{33446}{2475}$ & 0 \\
\hline$I_{49}^{v}$ & $\frac{9556}{1485}$ & $\frac{31057}{4950}$ & $\frac{38224}{2475}$ & 0 \\
\hline$I_{50}^{v}$ & $-\frac{16532}{1485} \tau_{a, b}+\frac{1403}{135} \tau_{a, c}$ & $-\frac{83233}{11880}$ & $-\frac{58961}{5940}$ & 0 \\
\hline$I_{51}^{v}$ & $\frac{9556}{1485} \tau_{a, b}^{2}$ & $\frac{33309827}{2673000}$ & $-\frac{74059}{13500}$ & 0 \\
\hline$I_{52}^{v}$ & $\frac{9556}{1485} \tau_{a, b}^{2}$ & $-\frac{1048771}{148500}$ & $-\frac{1822807}{74250}$ & $-\frac{2389}{2970}$ \\
\hline$I_{53}^{v}$ & $-\frac{19112}{1485} \tau_{a, b}-\frac{19112}{1485} \tau_{a, c}$ & $\frac{303403}{74250}$ & $\frac{1335451}{37125}$ & 0 \\
\hline$I_{54}^{v}$ & $-\frac{17036}{1485} \tau_{a, b}$ & $-\frac{8518}{1485}$ & $-\frac{34072}{1485}$ & $\frac{8518}{297}$ \\
\hline$I_{55}^{v}$ & $\begin{array}{l}-\frac{19112}{1485} \\
140\end{array}$ & $\frac{962767}{20700}$ & $\frac{398963}{4950}$ & $\begin{array}{c}291 \\
0\end{array}$ \\
\hline$I_{56}^{v}$ & $\frac{19112}{1485}$ & $\begin{array}{l}29100 \\
-\frac{16723}{1980}\end{array}$ & $\begin{array}{r}4950 \\
-\frac{2389}{110}\end{array}$ & 0 \\
\hline$I_{57}^{v}$ & $\begin{array}{r}1400 \\
-\frac{934}{99}\end{array}$ & $-\frac{91999}{4050}$ & $-\frac{38761}{825}$ & 0 \\
\hline$I_{58}^{v}$ & $-\frac{2711}{5940} \tau_{a, d}+\frac{263}{540} \tau_{b, c}$ & $\begin{array}{l}49141 \\
89141\end{array}$ & $\begin{array}{r}825 \\
-11527 \\
1\end{array}$ & 0 \\
\hline$I_{59}^{v}$ & $-\frac{2711}{2970}$ & $\begin{array}{r}19800 \\
-35243 \\
\end{array}$ & $\begin{array}{r}2475 \\
-46087 \\
\end{array}$ & 0 \\
\hline$I_{60}^{v}$ & $-\frac{9556}{1485} \tau_{a, b}$ & $-\frac{54947}{-0400}$ & $\begin{array}{r}12350 \\
-\frac{74059}{20700} \\
\end{array}$ & 0 \\
\hline$I_{61}^{v}$ & $\frac{1868}{105} \tau_{a b}$ & $\begin{array}{r}587300 \\
8873 \\
\end{array}$ & $\begin{array}{r}29680 \\
-1868 \\
\end{array}$ & $\Omega$ \\
\hline$I^{v}$ & $\begin{array}{c}495 \\
9556\end{array}$ & $\begin{array}{r}4125 \\
14095\end{array}$ & $\begin{array}{r}4125 \\
174309\end{array}$ & $U$ \\
\hline${ }^{1} 62$ & $\frac{5050}{1485}$ & $\frac{1+051}{14850}$ & $\frac{1450}{7425}$ & 0 \\
\hline$I_{63}^{v}$ & $\frac{1868}{495} \tau_{a, b}$ & $\frac{34091}{12375}$ & $-\frac{31756}{12375}$ & $-\frac{934}{495}$ \\
\hline$I_{64}^{v}$ & $\frac{467}{495} \tau_{a, b}^{2}$ & $-\frac{467}{600}$ & $\frac{17279}{4950}$ & $-\frac{467}{1980}$ \\
\hline$I_{65}^{v}$ & $\frac{14101}{1980}$ & $\frac{183313}{6600}$ & $\frac{49404}{825}$ & 0 \\
\hline$I_{66}^{v}$ & $-\frac{8588}{1485}$ & $-\frac{916769}{74250}$ & $-\frac{382166}{12375}$ & 0 \\
\hline$I_{67}^{v}$ & $\frac{1868}{495} \tau_{a, b}^{2}$ & $\frac{1401}{550}$ & $\frac{3736}{825}$ & $\frac{467}{495}$ \\
\hline$I_{68}^{v}$ & $\frac{1868}{495} \tau_{a, b}^{2}$ & $\frac{467}{450}$ & $\frac{1868}{825}$ & $\frac{467}{165}$ \\
\hline$I_{69}^{v}$ & $\frac{4778}{1485} \tau_{a, b}^{2}$ & $-\frac{3843901}{297000}$ & $\frac{231733}{148500}$ & $\frac{54947}{2970}$ \\
\hline Total & - & $\frac{23}{2}$ & 23 & $\frac{23}{2}$ \\
\hline
\end{tabular}


[1] L. Brink, J. H. Schwarz and J. Scherk, Nucl. Phys. B 121, 77 (1977);

F. Gliozzi, J. Scherk and D. I. Olive, Nucl. Phys. B 122, 253 (1977).

[2] C. Anastasiou, Z. Bern, L. J. Dixon and D. A. Kosower, Phys. Rev. Lett. 91, 251602 (2003) [hep-th/0309040];

Z. Bern, L. J. Dixon and V. A. Smirnov, Phys. Rev. D 72, 085001 (2005) [hep-th/0505205];

N. Beisert, B. Eden and M. Staudacher, J. Stat. Mech. 0701, P01021 (2007) [hep-th/0610251];

L. F. Alday and J. Maldacena, JHEP 0706, 064 (2007) [arXiv:0705.0303 [hep-th]];

F. Cachazo, M. Spradlin and A. Volovich, Phys. Rev. D 74, 045020 (2006) [arXiv:hepth/0602228];

Z. Bern, M. Czakon, D. A. Kosower, R. Roiban and V. A. Smirnov, Phys. Rev. Lett. 97, 181601 (2006) [arXiv:hep-th/0604074];

M. Spradlin, A. Volovich and C. Wen, Phys. Rev. D 78, 085025 (2008) [arXiv:0808.1054 [hep-th]].

[3] L. F. Alday and R. Roiban, Phys. Rept. 468, 153 (2008) [arXiv:0807.1889 [hep-th]];

N. Arkani-Hamed, J. L. Bourjaily, F. Cachazo, S. Caron-Huot and J. Trnka, JHEP 1101, 041 (2011) [arXiv:1008.2958 [hep-th]];

J. M. Henn, J. Phys. A 44, 454011 (2011) [arXiv:1103.1016 [hep-th]];

Z. Bern and Y.-t. Huang, J. Phys. A 44, 454003 (2011) [arXiv:1103.1869 [hep-th]];

T. Adamo, M. Bullimore, L. Mason and D. Skinner, J. Phys. A 44, 454008 (2011) [arXiv:1104.2890 [hep-th]];

L. J. Dixon, J. Phys. A 44, 454001 (2011) [arXiv:1105.0771 [hep-th]].

[4] J. M. Drummond, J. Henn, V. A. Smirnov and E. Sokatchev, JHEP 0701, 064 (2007) [hepth/0607160];

J. M. Drummond, G. P. Korchemsky and E. Sokatchev, Nucl. Phys. B 795, 385 (2008) [arXiv:0707.0243 [hep-th]];

A. Brandhuber, P. Heslop and G. Travaglini, Nucl. Phys. B 794, 231 (2008) [arXiv:0707.1153 [hep-th]];

J. M. Drummond, J. Henn, G. P. Korchemsky and E. Sokatchev, Nucl. Phys. B 795, 52 (2008) [arXiv:0709.2368 [hep-th]]; Nucl. Phys. B 826, 337 (2010) [arXiv:0712.1223 [hep-th]].

[5] Z. Bern, M. Czakon, L. J. Dixon, D. A. Kosower and V. A. Smirnov, Phys. Rev. D 75, 085010 (2007) [hep-th/0610248].

[6] J. M. Drummond, J. M. Henn and J. Plefka, JHEP 0905, 046 (2009) [arXiv:0902.2987 [hepth]];

T. Bargheer, N. Beisert, W. Galleas, F. Loebbert and T. McLoughlin, JHEP 0911, 056 (2009) [arXiv:0905.3738 [hep-th]];

N. Beisert, J. Henn, T. McLoughlin and J. Plefka, JHEP 1004, 085 (2010) [arXiv:1002.1733 [hep-th]].

[7] N. Beisert et al., arXiv:1012.3982 [hep-th].

[8] Z. Bern, L. J. Dixon, D. C. Dunbar, M. Perelstein and J. S. Rozowsky, Nucl. Phys. B 530, 401 (1998) [hep-th/9802162].

[9] Z. Bern, J. J. Carrasco, L. J. Dixon, H. Johansson, D. A. Kosower and R. Roiban, Phys. Rev. Lett. 98, 161303 (2007) [hep-th/0702112].

[10] Z. Bern, J. J. Carrasco, L. J. Dixon, H. Johansson and R. Roiban, Phys. Rev. Lett. 103, 
081301 (2009) [arXiv:0905.2326 [hep-th]].

[11] Z. Bern, J. J. M. Carrasco, L. J. Dixon, H. Johansson and R. Roiban, Phys. Rev. D 82, 125040 (2010) [arXiv:1008.3327 [hep-th]].

[12] E. Cremmer and B. Julia, Phys. Lett. B 80, 48 (1978); Nucl. Phys. B 159, 141 (1979).

[13] Z. Bern, J. J. M. Carrasco and H. Johansson, arXiv:0902.3765 [hep-th];

H. Nicolai, Physics 2, 70 (2009);

R. P. Woodard, Rept. Prog. Phys. 72, 126002 (2009) [arXiv:0907.4238 [gr-qc]];

L. J. Dixon, arXiv:1005.2703 [hep-th];

H. Elvang, D. Z. Freedman and M. Kiermaier, J. Phys. A 44, 454009 (2011) [arXiv:1012.3401 [hep-th]];

Z. Bern, J. J. Carrasco, L. Dixon, H. Johansson and R. Roiban, arXiv:1103.1848 [hep-th].

[14] S. Deser, J. H. Kay and K. S. Stelle, Phys. Rev. Lett. 38, 527 (1977).

[15] S. Ferrara and B. Zumino, Nucl. Phys. B 134, 301 (1978).

[16] S. Deser and J. H. Kay, Phys. Lett. B 76, 400 (1978).

[17] P. S. Howe and U. Lindström, Nucl. Phys. B 181, 487 (1981).

[18] R. E. Kallosh, Phys. Lett. B 99, 122 (1981).

[19] H. Elvang and M. Kiermaier, JHEP 1010, 108 (2010) [arXiv:1007.4813 [hep-th]].

[20] G. Bossard, C. Hillmann and H. Nicolai, JHEP 1012, 052 (2010) [arXiv:1007.5472 [hep-th]].

[21] G. Bossard, P. S. Howe and K. S. Stelle, JHEP 1101, 020 (2011) [arXiv:1009.0743 [hep-th]].

[22] N. Beisert, H. Elvang, D. Z. Freedman, M. Kiermaier, A. Morales and S. Stieberger, Phys. Lett. B 694, 265 (2010) [arXiv:1009.1643 [hep-th]].

[23] G. Bossard, P. S. Howe, K. S. Stelle and P. Vanhove, Class. Quant. Grav. 28, 215005 (2011) [arXiv:1105.6087 [hep-th]].

[24] M. T. Grisaru and W. Siegel, Nucl. Phys. B 201, 292 (1982) [Erratum-ibid. B 206, 496 (1982)].

[25] M. B. Green, J. G. Russo and P. Vanhove, JHEP 1006, 075 (2010) [arXiv:1002.3805 [hep-th]].

[26] J. Björnsson and M. B. Green, JHEP 1008, 132 (2010) [arXiv:1004.2692 [hep-th]];

J. Bjornsson, JHEP 1101, 002 (2011) [arXiv:1009.5906 [hep-th]].

[27] R. Kallosh and P. Ramond, arXiv:1006.4684 [hep-th].

[28] G. Chalmers, hep-th/0008162;

M. B. Green, J. G. Russo and P. Vanhove, JHEP 0702, 099 (2007) [hep-th/0610299];

Z. Bern, L. J. Dixon and R. Roiban, Phys. Lett. B 644, 265 (2007) [arXiv:hep-th/0611086];

M. B. Green, J. G. Russo and P. Vanhove, Phys. Rev. Lett. 98, 131602 (2007) [hepth/0611273];

R. Kallosh, JHEP 1012, 009 (2010) [arXiv:1009.1135 [hep-th]]; arXiv:1103.4115 [hep-th]; JHEP 1106, 073 (2011) [arXiv:1104.5480 [hep-th]].

[29] Z. Bern, J. J. M. Carrasco and H. Johansson, Phys. Rev. D 78, 085011 (2008) [arXiv:0805.3993 [hep-ph]].

[30] Z. Bern, J. J. M. Carrasco and H. Johansson, Phys. Rev. Lett. 105, 061602 (2010) [arXiv:1004.0476 [hep-th]].

[31] H. Kawai, D. C. Lewellen and S.-H. H. Tye, Nucl. Phys. B 269, 1 (1986);

Z. Bern, Living Rev. Rel. 5, 5 (2002) [gr-qc/0206071].

[32] J. J. M. Carrasco and H. Johansson, J. Phys. A 44, 454004 (2011) [arXiv:1103.3298 [hep-th]].

[33] J. J. M. Carrasco and H. Johansson, Phys. Rev. D 85, 025006 (2012) [arXiv:1106.4711 [hepth]].

[34] Z. Bern, J. J. Carrasco, T. Dennen, Y.-t. Huang and H. Ita, Phys. Rev. D 83, 085022 (2011) [arXiv:1010.0494 [hep-th]]. 
[35] C. Cheung and D. O'Connell, JHEP 0907, 075 (2009) [arXiv:0902.0981 [hep-th]];

T. Dennen, Y.-t. Huang and W. Siegel, JHEP 1004, 127 (2010) [arXiv:0910.2688 [hep-th]].

[36] G. 't Hooft and M. J. G. Veltman, Nucl. Phys. B 44, 189 (1972).

[37] L. F. Alday, J. M. Henn, J. Plefka and T. Schuster, JHEP 1001, 077 (2010) [arXiv:0908.0684 [hep-th]];

J. M. Henn, S. G. Naculich, H. J. Schnitzer and M. Spradlin, JHEP 1004, 038 (2010) [arXiv:1001.1358 [hep-th]]; JHEP 1008, 002 (2010) [arXiv:1004.5381 [hep-th]].

[38] See the ancillary file for the arXiv version of this manuscript.

[39] Z. Bern, L. J. Dixon, D. C. Dunbar and D. A. Kosower, Nucl. Phys. B 425, 217 (1994) [hepph/9403226]; Nucl. Phys. B 435, 59 (1995) [hep-ph/9409265];

Z. Bern, L. J. Dixon and D. A. Kosower, Ann. Rev. Nucl. Part. Sci. 46, 109 (1996) [hep$\mathrm{ph} / 9602280]$.

[40] R. Britto, F. Cachazo and B. Feng, Nucl. Phys. B 725, 275 (2005) [hep-th/0412103].

[41] Z. Bern, J. J. M. Carrasco, H. Johansson and D. A. Kosower, Phys. Rev. D 76, 125020 (2007) [arXiv:0705.1864 [hep-th]].

[42] D. Zhu, Phys. Rev. D 22, 2266 (1980);

C. J. Goebel, F. Halzen and J. P. Leveille, Phys. Rev. D 23, 2682 (1981).

[43] S.-H. H. Tye and Y. Zhang, JHEP 1006, 071 (2010) [arXiv:1003.1732 [hep-th]];

N. E. J. Bjerrum-Bohr, P. H. Damgaard, T. Sondergaard and P. Vanhove, JHEP 1006, 003 (2010) [arXiv:1003.2403 [hep-th]];

[44] J. Broedel and J. J. M. Carrasco, Phys. Rev. D 84, 085009 (2011) [arXiv:1107.4802 [hep-th]].

[45] Z. Bern, T. Dennen, Y.-t. Huang and M. Kiermaier, Phys. Rev. D 82, 065003 (2010) [arXiv:1004.0693 [hep-th]].

[46] R. Monteiro and D. O'Connell, JHEP 1107, 007 (2011) [arXiv:1105.2565 [hep-th]].

[47] M. Kiermaier, presented at Amplitudes 2010, http://www.strings.ph.qmul.ac.uk/ theory/Amplitudes2010;

N. E. J. Bjerrum-Bohr, P. H. Damgaard, T. Sondergaard and P. Vanhove, JHEP 1101, 001 (2011) [arXiv:1010.3933 [hep-th]];

C. R. Mafra, O. Schlotterer and S. Stieberger, JHEP 1107, 092 (2011) [arXiv:1104.5224 [hepth]].

[48] B. Feng, R. Huang and Y. Jia, Phys. Lett. B 695, 350 (2011) [arXiv:1004.3417 [hep-th]];

Y.-X. Chen, Y.-J. Du and B. Feng, JHEP 1102, 112 (2011) [arXiv:1101.0009 [hep-th]];

Y.-J. Du, B. Feng and C.-H. Fu, JHEP 1108, 129 (2011) [arXiv:1105.3503 [hep-th]].

[49] N. E. J. Bjerrum-Bohr, P. H. Damgaard and P. Vanhove, Phys. Rev. Lett. 103, 161602 (2009) [arXiv:0907.1425 [hep-th]];

S. Stieberger, arXiv:0907.2211 [hep-th].

[50] C. R. Mafra, O. Schlotterer and S. Stieberger, arXiv:1106.2645 [hep-th]; arXiv:1106.2646 [hepth].

[51] Z. Bern and T. Dennen, Phys. Rev. Lett. 107, 081601 (2011) [arXiv:1103.0312 [hep-th]].

[52] M. B. Green, J. H. Schwarz and L. Brink, Nucl. Phys. B 198, 474 (1982).

[53] Z. Bern, J. S. Rozowsky and B. Yan, Phys. Lett. B 401, 273 (1997) [hep-ph/9702424].

[54] Z. Bern, L. J. Dixon and D. A. Kosower, JHEP 0001, 027 (2000) [hep-ph/0001001].

[55] Z. Bern, J. J. M. Carrasco, L. J. Dixon, H. Johansson and R. Roiban, Phys. Rev. D 78, 105019 (2008) [arXiv:0808.4112 [hep-th]].

[56] Z. Bern, C. Boucher-Veronneau and H. Johansson, Phys. Rev. D 84, 105035 (2011) [arXiv:1107.1935 [hep-th]]. 
[57] D. C. Dunbar, J. H. Ettle and W. B. Perkins, Phys. Rev. D 83, 065015 (2011) [arXiv:1011.5378 [hep-th]].

[58] C. Boucher-Veronneau and L. J. Dixon, JHEP 1112, 046 (2011) [arXiv:1110.1132 [hep-th]].

[59] S. G. Naculich, H. Nastase and H. J. Schnitzer, JHEP 1201, 041 (2012) [arXiv:1111.1675 [hep-th]].

[60] S. Laporta, Int. J. Mod. Phys. A 15, 5087 (2000) [hep-ph/0102033].

[61] E. J. Martinec, Phys. Lett. B 171, 189 (1986).

[62] M. T. Grisaru, W. Siegel and M. Roček, Nucl. Phys. B 159, 429 (1979);

N. Seiberg, Phys. Lett. B 318, 469 (1993) [hep-ph/9309335].

[63] P. S. Howe and K. S. Stelle, Phys. Lett. B 554, 190 (2003) [hep-th/0211279].

[64] N. Berkovits, M. B. Green, J. G. Russo and P. Vanhove, JHEP 0911, 063 (2009) [arXiv:0908.1923 [hep-th]].

[65] I. Robinson, unpublished;

L. Bel, Acad. Sci. Paris, Comptes Rend. 247:1094 (1958) and 248:1297 (1959).

[66] D. Z. Freedman and E. Tonni, JHEP 1104, 006 (2011) [arXiv:1101.1672 [hep-th]].

[67] A. A. Vladimirov, Theor. Math. Phys. 43, 417 (1980) [Teor. Mat. Fiz. 43, 210 (1980)].

[68] N. Marcus and A. Sagnotti, Nuovo Cim. A 87, 1 (1985).

[69] G. Bossard, P. S. Howe and K. S. Stelle, Phys. Lett. B 682, 137 (2009) [arXiv:0908.3883 [hep-th]];

G. Bossard, P. S. Howe, U. Lindström, K. S. Stelle and L. Wulff, JHEP 1105, 021 (2011) [arXiv:1012.3142 [hep-th]].

[70] K. G. Chetyrkin, A. L. Kataev and F. V. Tkachov, Nucl. Phys. B 174, 345 (1980).

[71] R. N. Lee and V. A. Smirnov, private communication.

[72] R. N. Lee, Nucl. Phys. B 830, 474 (2010) [arXiv:0911.0252 [hep-ph]]; Nucl. Phys. Proc. Suppl. 205-206, 135 (2010) [arXiv:1007.2256 [hep-ph]].

[73] O. V. Tarasov, Phys. Rev. D 54, 6479 (1996) [arXiv:hep-th/9606018].

[74] R. N. Lee, A. V. Smirnov and V. A. Smirnov, JHEP 1004, 020 (2010) [arXiv:1001.2887 [hepph]]; Nucl. Phys. Proc. Suppl. 205-206, 308 (2010) [arXiv:1005.0362 [hep-ph]]; Eur. Phys. J. C 71, 1708 (2011) [arXiv:1103.3409 [hep-th]]; Nucl. Phys. B 856, 95 (2012) [arXiv:1108.0732 [hep-th]];

R. N. Lee and V. A. Smirnov, JHEP 1102, 102 (2011) [arXiv:1010.1334 [hep-ph]].

[75] K. G. Chetyrkin and F. V. Tkachov, Phys. Lett. B 114, 340 (1982);

K. G. Chetyrkin and V. A. Smirnov, Phys. Lett. B 144, 419 (1984).

[76] K. G. Chetyrkin and F. V. Tkachov, Nucl. Phys. B 192, 159 (1981).

[77] C. Anastasiou and A. Lazopoulos, JHEP 0407, 046 (2004) [hep-ph/0404258]. 